\title{
THE SUMMER MEETING IN SEATTLE
}

The sixty-first Summer Meeting of the American Mathematical Society was held from Tuesday, August 21, through Friday, August 24, 1956, at the University of Washington, Seattle, Washington. Other organizations meeting in Seattle at about the same time were the Mathematical Association of America, the Institute of Mathematical Statistics, the Biometric Society, and the Econometric Society.

Over 580 people registered for the meeting, including about 370 members of the Society.

Professor Salomon Bochner of Princeton University delivered the Colloquium Lectures on Harmonic analysis and probability, with Professors Richard Brauer, L. V. Ahlfors, C. B. Allendoerfer, and R. S. Phillips presiding.

By invitation of the Committee to Select Hour Speakers for Annual and Summer Meetings, the Society was addressed by Professors C. B. Allendoerfer, Kenkichi Iwasawa, and Sarvadaman Chowla, their topics being (respectively) Some recent advances in differential geometry in the large, $A$ theorem on Abelian groups and its application in algebraic number theory, and Difference sets and finite geometries. They were introduced (respectively) by Professors C. O. Oakley, Deane Montgomery and R. H. Bruck.

There was a Session of Invited Papers in Applied Mathematics, presided over by Dr. R. E. Gaskell. Speakers were Professors Bernard Friedman, P. L. Chambré, J. W. Carr III, Dr. William Nachbar, and Dr. R. L. Brock. Abstracts of their papers appear in this report along with those of other papers in Applied Mathematics.

A session for contributed papers in Probability and Statistics was held jointly with the Institute of Mathematical Statistics, Dr. I. J. Good presiding. There were thirteen other sessions for contributed papers, presided over by Dr. F. J. Weyl and Professors R. V. Churchill, J. L. Kelley, R. P. Dilworth, M. F. Smiley, Casper Goffman, R. D. James, Edwin Hewitt, R. A. Beaumont, H. F. Bohnenblust, G. B. Price, F. A. Valentine, and M. M. Day.

From the smooth progress of the meeting and the fine recreation and entertainment provided, it was evident that the Committee on Arrangements had worked hard and well. In this connection, the Society owes special thanks to Professor A. E. Livingston, Chairman of the Committee. 
The Council met on Tuesday evening, August 21, 1956.

The Secretary announced the election of the following ninety-eight persons to ordinary membership in the Society:

Professor Bernhard Banaschewski, McMaster University;

Dr. William D. Barcus, Jr., Brown University;

Mrs. Anne Breese Barnes, University of Texas;

Professor Clyde Raymond Barrow, Westminster College;

Mr. Kenneth Reynold Blake, Marquette University;

Mr. James Robert Boyd, Chance Vaught Aircraft, Dallas, Texas;

Mr. Arthur Eugene Bragg, Tuskegee Institute;

Mr. Donald George Brennan, Massachusetts Institute of Technology;

Miss Lena Celeste Brown, Southern University;

Dr. James Floyd Carpenter, Boeing Airplane Company, Seattle, Washington;

Mr. Louis Child, New Mexico College of Agriculture and Mechanic Arts;

Mr. Charles Cyprian Chouteau, University of Wichita;

Mr. Robert William Cowan, University of Georgia;

Mrs. Alice Roth Crilly, Mount St. Mary's College;

Professor Geoffrey Crofts, Occidental College;

Professor Jacob Cukierman, Acadia University;

Mr. William M. Cunnea, University of Chicago;

Professor Frances Elizabeth Davis, University of Hawaii;

Professor Mamie Myrtis Davis, Wesleyan College;

Mr. Richard Thomas Dillon, University of Oregon;

Mrs. Elsie Phelps Elder, Washington University;

Sister M. Barbara Ann Foos, SSJ, Nazareth College;

Mr. Donald Roy Fredkin, New York University;

Mr. Eugene John Gehrig, Northwestern University;

Mr. Charles Joseph Gentry, University of Florida;

Dr. Bruce Gilchrist, Syracuse University;

Dr. Allen A. Goldstein, Convair-Astronautics, San Diego, California;

Mr. George Ross Grainger, Convair-Astronautics, San Diego, California;

Professor Mauritz Leland Granberg, Sioux Falls College;

Mr. Robert Karl Gruenewald, Washington University;

Mr. Herschel Nixon Hadley, Naval Powder Factory, Indian Head, Maryland;

Mr. Walter Elliott Haggerty, Louisiana State University;

Dr. Dilla Hall, Southern Illinois University;

Mr. Robert L. Helmbold, Carnegie Institute of Technology;

Mr. Michael Herschorn, McGill University;

Mr. Robert George Heyneman, University of California, Berkeley;

Professor William Floyd Hill, Howard Payne College;

Mr. Lewis Eugene Hulbert, Battelle Memorial Institute, Columbus 1, Ohio;

Mr. Lambert S. Joel, National Bureau of Standards, Washington 25, D. C.;

Mr. Elgy Sibley Johnson, The Joel E. Spingarn High School, Washington, D. C.;

Miss Katherine Everts Kellner, University of Illinois;

Mr. Merrill Jay Kemp, University of Utah;

Mr. James Edward Kiefer, University of Minnesota;

Mr. Donald Ross King, Rutgers University;

Mr. Ralph Ellis Kleinman, University of Michigan;

Dr. Herbert Knothe, Holloman Air Development Center, Holloman, New Mexico; 
Mr. George Richard Kuhn, St. Louis University;

Mr. Donald Irvine Kurth, University of Minnesota;

Mr. John Grieg Leghorn, University of Colorado;

Mr. Hugh Lloyd Lewis, Southwest Texas State Teachers College;

Mr. Arthur Libenson, General Electric Company, Ithaca, New York;

Mr. Irving J. Lieberman, Litton Industries, Beverly Hills, California;

Dr. Stuart P. Lloyd, Bell Telephone Laboratories, Inc.;

Dr. Wilhelmus Antonius J. Luxemburg, University of Toronto;

Mr. Rogers William Martin, School Board, Wilmington, Delaware;

Professor Joseph Courtney McCully, University of Rhode Island;

Mr. E. Gregory McNiel, DePaul University;

Professor Jack R. Meagher, Western Michigan College;

Mr. Richard Frederick Meyer, Arthur D. Little, Inc., Cambridge, Massachusetts;

Mr. R. A. Moreland, Jr., Texas Technological College;

Mr. Florio Joseph Moretti, Fairfield University;

Mr. Herbert Alfred Morrissey, Reynolds Metals Company, Sheffield, Alabama;

Professor Charles E. Moulton, Shurtleff College;

Sister Rose Marian Mulligan, O. S. F., Ladycliff College;

Mr. Patrick Lawrence O'Dea, White Sands Proving Ground, New Mexico;

Professor George Harry Pavlakos, Elmhurst College;

Dr. Rudolph William Preisendorfer, Scripps Institute of Oceanography, San Diego, California;

Mr. Robert Pruitt, University City High School, University City, Missouri;

Mr. Ronald Pyke, University of Washington;

Mr. Gerhard Rayna, Lehigh University;

Professor John Reckzeh, State Teachers College, Jersey City, New Jersey;

Professor Thelma Austin Rice, Alabama State College;

Mr. John Astwood Riley, Boston College;

Comdr. Horace Leland de Rivera, (U.S.N., retired), Northeastern University;

Mr. James Noel Rogers, University of Wisconsin;

Mr. Robert Rosen, Columbia University;

Mr. Adolph Andrew Rutoskey, Fort McPherson, Georgia;

Professor Hans J. Sagan, Montana State College;

Miss Lily H. Seshu, University of Illinois;

Dr. Joseph Richard Sligo, University of Nevada;

Miss Emma D. Breedlove Smith, South Carolina State College;

Mr. Lloyd Benton Smith, Jr., Louisiana State University;

Mr. William Frank Steele, Heidelberg College;

Rev. Thomas James Taylor, St. Ambrose College;

Mr. J. Richard Tessmer, Massachusetts Institute of Technology;

Professor Robert Harry Thompson, Sterling College;

Sister M. Denis Treacy, O.S.F., The Assisium, New York, New York;

Professor Donald Robert Truax, University of Kansas;

Mr. Edward J. Tully, Jr., Master Beach, New York;

Professor Mary K. Tulock, California State Polytechnic College;

Sister Mary Robert Von Wolff, O.P., St. Mary's Dominican College;

Mr. Max Leslie Weiss, Cornell University;

Mr. Frederick R. White, University of Buffalo;

Mr. Jay Charles Williams, University of Kansas;

Dr. Joseph Clement Wilson, Redstone Arsenal, Huntsville, Alabama; 
Professor George Anderson Yates, Tennessee Wesleyan College;

Miss Sallyann Elizabeth Zimm, University of Illinois;

Professor Geraldyne Pierce Zimmerman, South Carolina State College.

It was reported that the following four persons had been elected to membership on nomination of institutional members as indicated:

Haverford College: Mr. Alexander Hamilton Frey, Jr.

University of Missouri: Mr. Billy J. Attebery

New York University: Mr. Martin Schechter

Stanford University: Mr. Richard Lee Van de Wetering.

The Secretary reported that the following had been admitted to the Society in accordance with reciprocity agreements with various mathematical organizations: Deutsche-Mathematiker-Vereinigung: Dr. Artur Bergmann, Ricarda Huch Schule, Professor E. F. Peschl, Mathematisches Institut der Universität, and Dr. Hans-Egon Richert, Mathematisches Institut der Universität; Société Mathématique de France: Professor Henri Eyraud, University of Lyon, Professor István Fáry, University of Montreal, and Dr. František Vyčichlo, University of Prague.

The following appointments by the President were reported: as a Program Committee for a Summer Seminar in Applied Mathematics to be held in 1957: Paul Garabedian, A. S. Householder, Mark Kac, R. E. Langer, C. C. Lin, M. H. Martin, Chairman, William Prager, and J. J. Stoker; as a Committee on Arrangements for the 1956 Annual Meeting to be held at the University of Rochester: Edward Batho, Dorothy Bernstein, H. M. Gehman, Norman Gunderson, Hewitt Kenyon, Robert MacDowell, Walter Rudin, Chairman, and R. D. Schafer; as a Committee on Arrangements for a meeting to be held November 30-December 1 at the University of Kentucky: V. F. Cowling, Chairman, H. H. Downing, J. C. Eaves, J. G. Horne, Frank Levin, S. E. Pence, J. H. Roberts, and W. C. Royster; as a member of the Committee on Translations for a period of three years beginning July 1, 1956: J. V. Wehausen; as Chairman of the Committee on Translations for a period of one year beginning July 1, 1956: Hans Samelson. (Committee now consists of R. E. Bellman, R. P. Boas, Jr., Irving Kaplansky, Hans Samelson, Chairman, and J. V. Wehausen); as members of the Organizing Committee for Summer Institutes for three years beginning July 1, 1956: R. H. Bing and H. S. M. Coxeter; as Chairman of the Organizing Committee for Summer Institutes for a period of one year beginning July 1, 1956: Edwin Hewitt. (Committee now consists of R. H. Bing, H. S. M. Coxeter, Nelson Dunford, Edwin Hewitt, Chairman, E. R. Kolchin, and J. J. Stoker); as a delegate to the Fourth Congress of Roumanian 
Mathematicians, May 27-June 4, 1956: D. G. Bourgin, as delegates to the International Symposium on Algebraic Topology held in Mexico City, August 6-September 1, 1956: Samuel Eilenberg, W. Hurewicz, and N. E. Steenrod.

The Secretary reported that Professors C. B. Allendoerfer, Kenkichi Iwasawa, and S. Chowla had accepted invitations to deliver hour addresses at the Summer Meeting of 1956 in Seattle, Washington; that Professor Bertram Yood will address the Society at the meeting in Pasadena, California, November 17, 1956; that Professor J.-P. Serre will address the Society at the meeting to be held at the Massachusetts Institute of Technology on October 27, 1956; that Professor George Piranian will address the Society at the meeting to be held at Northwestern University on November 23-24, 1956; that Professor O. G. Harrold, Jr. will address the Society at the meeting to be held at the University of Kentucky, November 30-December 1, 1956; that Professor H. J. Muller has accepted an invitation to deliver the Gibbs Lecture in January, 1958; that Professor J. L. Doob has accepted an invitation to deliver the Colloquium Lectures in 1959 (the lectures for 1958 have been omitted); that Professors P. R. Halmos, S. C. Kleene, W. V. Quine, J. B. Rosser, Chairman, and Alfred Tarski have been named as an Invitations Committee for the 1957 Summer Institute; that Professor W. S. Massey will replace Professor Samuel Eilenberg on the Transactions and Memoirs Editorial Committee for the period June 1, 1956 to July 15, 1957 and will serve as Chairman of this committee for the remainder of 1956, that Dr. S. H. Gould has been appointed Executive Editor of Mathematical Reviews for a term of one year beginning June, 1956.

The Council set the following dates and places of meetings: February 23, 1957 at Yale University; April 20, 1957 at the University of California at Berkeley; and January 28-30,1958 at the University of Cincinnati and the Sheraton-Gibson Hotel, Cincinnati, Ohio.

The Council voted to approve a Symposium on Applied Mathematics on the topic "Orbit Theory" to be held in conjunction with the meeting at New York University in April, 1957.

A business meeting of the Society was held at 10:00 a.m., August 23, 1956, with President Elect Brauer presiding. The Secretary reported briefly on the affairs of the Society.

The following amendments to the by-laws were adopted: Sections 9 and 10 of Article IX were amended to read as follows:

Section 9. An ordinary or contributing member shall receive the Bulletin and Proceedings as privileges of membership during each year for which his dues have been discharged, except that a member 
may substitute for the Proceedings, the Transactions or Mathematical Reviews by paying a premium established by the Trustees for this privilege.

Section 10. Ten dollars of the annual dues of those who receive the Bulletin and the Proceedings, or the Transactions or Mathematical Reviews in substitution for the Proceedings, under the provisions of this Article shall be allocated in payment therefor. Additionally, in the case of substitution of the Transactions or Mathematical Reviews for the Proceedings, the entire amount of the premium referred to in Section 9 shall also be so allocated.

A resolution of thanks to the host institution was adopted.

The abstracts of the papers follow. Those having the letter " $t$ " following the abstract number were read by title. Where a paper, presented in person, has more than one author, the symbol (p) follows the name of the author who presented it. Dr. E. H. Bareiss was introduced by Dr. Feodor Theilheimer, Professors Fryer and Helperin by Professor H. W. Ellis, Mr. R. P. Hunter by Professor P. M. Swingle, Professor P. W. M. John by Professor V. L. Klee, Jr., Professor Erwin Kreyszig by Professor Stefan Bergman, Dr. T. C. H. Li by Dr. H. E. Salzer, Mr. W. D. Maness by Dr. P. O. Bell, Mr. George Marsaglia by Professor V. L. Klee, Jr., Dr. Richard Montague by Dr. R. L. Vaught, Mr. R. M. Smullyan by Professor John McCarthy, and Dr. R. C. Thorne, by Professor H. F. Bohnenblust.

\section{Algebra ANd Theory of Numbers}

\section{N. C. Ankeny: Quadratic forms.}

A new proof of formula for the representation of a number as the sum of $n$ squares, $n \leqq 8$ is presented. Let $V_{n}(p)$ denote the $n$ dimensional Euclidean vector space over the finite field with $p$ elements, $p$ a rational prime, with the usual dot product and metric. Then count the number of non-null lines, a line being all scalar multiples of a vector, a null line being all multiples of a vector of zero length. By the use of Geometry of Numbers it is then shown that each null line gives $2 n$ solutions of our original problem. Conversely by Witt's theorem, almost every solution corresponds to a reflection with respect to a non-null line. The solutions left over are shown to correspond to certain isometrics in planes orthogonal to null-lines. This yields the formulae. (Received June 25, 1956.)

\section{5t. H. W. Becker: On the triangles of Bancroft Brown.}

These are pairs of Heronian triangles ( $H \AA$ ) $a, b, c$ and $a, b, c^{\prime}$ with 2 sides and the radius $r$ of the inscribed circle in common (Brownian triangles, $B \AA$ ). Brown gave 7 numerical solutions, (Math. Mag. vol. 29 (1956) pp. 275-276). \#1, 2, 4 generalize to (I) $r, a, b, c, c^{\prime}=2 m n\left(m^{2}-n^{2}\right), m^{4}+6 m^{2} n^{2}+n^{4}, 2\left(m^{2}+n^{2}\right)^{2},\left(3 m^{2}+n^{2}\right)\left(m^{2}-n^{2}\right)$, $m^{4}+14 m^{2} n^{2}+n^{4}$. \#3 generalizes to (II) $r, a, b, c, c^{\prime}=m n\left(m^{2}-n^{2}\right)\left(m^{2}-m n+n^{2}\right)$, $m^{6}+n^{6}, 2 m n\left(m^{4}+n^{4}\right),\left(m^{2}-m n+n^{2}\right)\left(m^{4}+m^{3} n-2 m^{2} n^{2}+m n^{3}+n^{4}\right),\left(m^{2}+n^{2}\right)\left(m^{4}+m^{2} n^{2}\right.$ 
$\left.+n^{4}\right)$. \#4 with both $\mathrm{S}$ obtuse (0-0) generalizes in (I) to any $m>4$ with $n=1$; (II) is type 0-0 when $m^{4}-3 m^{2} n^{2}+n^{4}$ is negative. Conjecture: In all $B \otimes, 6 \mid r$. It is evident from comparison of $r$ in (I) and (II) that they can yield no common numerical solution. They were arrived at by the problem solving principles of Polya (guessing!) based on consideration of the generators $e, f$ and $g, h$ of the component Pythagorean $\triangle$. The condition for $B S$ is that the quartic $e^{2} g^{2}(e h-f g)^{2}+4 f^{2} h^{2}\left(e^{2} g^{2}+e f g h-f^{2} h^{2}\right)$ $=\square$. Very likely no complete solution, in polynomials of any finite degree or weight, can exist. For every $B \triangle$ pair, there is a third "algebraic triangle" with the same $r, a, b$, but third "side" $c$ " negative. What is the density of $B \AA$ among $H \&$ ? What types of $H \mathrm{~S}$ cannot be $B \Theta$ ? (Received July 6, 1956.)

596t. Gerald Berman and R. J. Silverman: Algebraic systems with n-operations.

A $\Gamma_{s}$-system is a system $\left\{S, \sigma_{1}, \sigma_{2}, \cdots, \sigma_{s}\right\}$ where $S$ is a nonempty set and $\sigma_{i}$ is a mapping from a nonempty subset $S_{i}$ of $S \times S$ to $S, i=1,2, \cdots, s$. A $\Gamma_{0}$-system is a system $\{S\}$. A $\Gamma_{s}(n)$-system is a $\Gamma_{s+n}$-system with the additional properties (i) $S_{i}$ $=S \times S, i>s$, (ii) $\sigma_{i}$ is associative, $i>s$, and (iii) $\sigma_{i}$ is left-distributive with respect to $\sigma_{j}$ for $s>i>j$. It is shown that (a) corresponding to any set $S$ and any integers $s$ and $n$ there exists a $\Gamma_{s}(n)$-system, (b) given any $\Gamma_{8}$-system $\mathcal{S}$ and any integer $n$, there exists a $\Gamma_{8}(n)$-system $\mathfrak{J}$ which contains a $\Gamma_{8}$-subsystem isomorphic to $\mathcal{S}$, such that if $S_{i}=S \times S$ for any $i$, the corresponding $T_{i}=T \times T$, (c) given any $\Gamma_{s}(n)$-system $\delta$ and any integer $m$, there exists a $\Gamma_{s}(n+m)$-system containing a $\Gamma_{s}(n)$-subsystem isomorphic to $\mathcal{S}$, and (d) given any $\Gamma_{s}(n)$ system $\mathcal{S}$ with set $S$, there exists a $\Gamma_{s}(n)$-system $\mathcal{J}$ with set $T$, having cardinality greater than that of $S$, such that $J$ contains a $\Gamma_{s}(n)$-subsystem isomorphic to $S$. (Received July 5,1956 .)

597. Gerald Berman and R. J. Silverman (p): Embedding of general algebraic systems into systems of transformations.

A $\Gamma_{s}(n)$-system is defined in the abstract Algebraic systems with n-operations. Several theorems are proved on the isomorphic embedding of a $\Gamma_{s}(n)$-system into a $\Gamma_{s}(n)$-system whose elements are transformations on a set, where the embedding satisfies certain restrictions. For example, it is shown that a $\Gamma_{s}(n)$-system $\left\{S, \sigma_{1}, \sigma_{2}, \cdots\right.$, $\left.\sigma_{s+n}\right\}$ can be embedded isomorphically into a $\Gamma_{s}(n)$-system $\left\{T^{n}, \tau_{1}^{n}, \tau_{2}^{n}, \cdots, \tau_{s+n}^{n}\right\}$ where (i) $T^{1}$ is the set of transformations on a set $T$ (T may be taken as the set of transformations on $S$ ) and $T^{k}$ is the set of transformations on $T^{k-1}$, (ii) $\tau_{i}^{n}$ corresponds to $\sigma_{i}, i=1,2, \cdots, s+n$, (iii) $\sigma_{i}$ is left-distributive with respect to $\sigma_{j}$ for $i>j, i>s$. These embedding theorems generalize many of the well known embedding theorems in algebra. As a special case, a ring can be embedded isomorphically into the endomorphisms of a group. It is not necessary to first embed the ring into a ring with identity as in the usual proof. The embedding theorems also subsume such theorems as: Every semi-group, (group, near-ring, semi-ring, associative near-ring) can be embedded isomorphically into the right multiplications on a semi-group (group, group, semi-group, loop). (Received July 5, 1956.)

\section{J. L. Brenner: New proof of a theorem of Taussky and Geirin-} ger.

If any principal minor of a matrix $A=\left(a_{i j}\right)$ is singular and if, for each $i,\left|a_{i i}\right|$ $\geqq \sum_{i}\left|a_{i j}\right|$, the principal minor of maximum dimension is singular. By renumbering the subscripts, the matrix can be reduced. (Received June 25, 1956.) 
599t. V. Bohun-Chudyniv: On methods of determining all types of Grave-Cayley algebras and some applications.

An oktonion algebra [see V. Bohun-Chudyniv, On a general method for constructing completely orthogonal $2^{k} \times 2^{k}$ squares by using orthogonal systems of $K$-nions, Proceedings of the International Congress of Mathematicians, 1954, Asmterdam, Holland, vol. II, pp. 281-282] is a Grave-Cayley algebra if the norm of the product of two arbitrary oktonions $P_{1}, P_{2}$, satisfy the condition (1): $N\left(P_{1} P_{2}\right)=N\left(P_{1}\right) N\left(P_{2}\right)$. At the present time only a very few types of Grave-Cayley algebras are known, differing from one another by their multiplication tables. Among them are: (I) J. T. Graves' type, communicated to W. R. Hamilton, Dec. 26, 1843 and Jan. 18, 1884, Proc. Royal Irish Academy (3) 1845-47; (II) A. Cayley's type, Phil. Mag., London, vol. XXX (1847) pp. 57-58; (III) L. E. Dickson's type, Linear algebras, Cambridge, 1914, p. 14; and others. The aims of this paper are: (A) to determine all types of oktonion algebras, the multiplication tables of which imply the condition (1), and including abovementioned algebras as separate cases; (B) to apply the multiplication table of algebras (A) for determining orthogonalizable Latin $7 \times 7$ Squares, and pertaining to each of them completely orthogonal Latin Squares of the same order (and constructing same). (Received July $5,1956$. )

\section{0t. R. H. Bruck: The structure of commutative Moufang loops.}

Let $G$ be a commutative Moufang loop with centre $Z(G)$, associator subloop $G^{\prime}$, inner mapping group $I(G)$, multiplication group $M(G)$. (1) If $G$ is finitely generated, each of $G / Z(G), G^{\prime}, I(G), M(G) / Z(M)$ is finite. (2) $G$ is locally centrally nilpotent. (3) $G / Z(G)$ and $G^{\prime}$ are locally finite loops of exponent 3. (4) $I(G)$ and $M(G) / Z(M)$ are locally finite 3-groups. (5) The following statements are equivalent: (i) $G$ is centrally nilpotent of (finite positive) class c; (ii) $I(G)$ is nilpotent of class $c-1$; (iii) $M(G)$ is nilpotent of class $2 c-1$. Statement (2) is proved in a form conjectured by Slaby and originally proved for $n \leqq 5$ by Slaby and the author (Bull. Amer. Math. Soc. Abstract 60-1-102): Every commutative Moufang loop which can be generared by $n$ elements $(n>1)$ is centrally nilpotent of class at most $n-1$. The class, $k(n)$, of the free commutative Moufang loop on $n$ free generators, satisfies the inequalities $1+[n / 2]$ $\leqq k(n) \leqq n-1$ for $n \geqq 3$. It is still unknown whether every infinitely generated commutative Moufang loop is (transfinitely) centrally nilpotent. (Received July 5, 1956.)

\section{1t. Eckford Cohen: Generalizations of the Euler $\phi$-function.}

New proofs of the characteristic properties of certain generalized totient functions are obtained. The Jordan function $J_{k}(n)$ and the author's generalized totient $\phi_{k}(n)$, (Bull. Amer. Math. Soc. Abstract 61-3-389), are considered. It is shown that both functions can be reformulated in terms of the $\phi$-function alone. On the basis of these formulas, the characteristic properties of $J_{k}(n)$ and $\phi_{k}(n)$ result easily from the corresponding properties of $\phi(n)$. (Received June $4,1956$.

\section{2t. Eckford Cohen: Cauchy products of certain types of arithmeti-} cal functions.

This paper is concerned primarily with approximation to the Cauchy product $\sum_{a-1}^{n} f(a) g(n-a)$ of certain types of almost periodic arithmetical functions $f(n), g(n)$. The method employed is based on Wintner's theory (Eratosthenian averages, 1943, Chapter 2) generalizing Ramanujan's trigonometric expansions, in connection with the author's orthogonality relation (Duke Math. J. vol. 19 (1952) p. 120) for the 
Ramanujan sum $c(n, r)$. Estimates associated with the mean values of arithmetical functions are also obtained, and the general theory is illustrated by means of divisor and totient functions. (Received June 4, 1956.)

\section{3t. Harvey Cohn: Some algebraic number theory estimates based on the Dedekind eta-function.}

For the pure cubic field, the zeta-function residue reduces to a finite expression involving the Dedekind eta-function. (See Dedekind, Crelle 121, 1900, pp. 40-123.) From this expression, using the Farey sub-division, the author obtains the estimate $h \log \epsilon=O\left(|d|^{1 / 2} \log |d| \log \log |d|\right)$, where $d$ is the field discriminant, $h$ is the class number, and $\epsilon(>1)$ is the fundamental unit. This is a slight improvement over Landau's more general $O\left(|d|^{1 / 2} \log |d|^{2}\right)$. From this follows the result $h=O\left(|d|^{1 / 2}\right.$ $\cdot \log \log |d|)$, in contrast to Landau's $O\left(|d|^{1 / 2} \log ^{2}|d|\right)$. Bounds on norms of certain modular invariants are included. (Received March 28, 1956.)

\section{4t. D. W. Crowe: A simple ordering for linear graphs. Preliminary report.}

For an arbitrary connected linear graph, $G$, a class of words $\{w(G)\}$ is described according to the following recipe: Beginning at some vertex of $G$ follow an arbitrary path (traversing each edge exactly once) recording the degree of each vertex as it occurs until either (a) a vertex of degree 1 is reached or (b) a vertex $u$ is reached which has already been recorded. In case (a) return to the first vertex at which there are edges not yet traversed and continue the process. In case (b) $u$ is the $n$th distinct recorded vertex having degree $d(u)$. Attach the subscript $n$ to this last $d(u)$ and proceed as in case (a). Continue the process until all edges have been traversed. If $G \neq G^{\prime}$ then $\{w(G)\} \cap\left\{w\left(G^{\prime}\right)\right\}=\phi$. In the usual lexicographic order let $W(G)$ be the smallest element of $\{w(G)\}$. Then define $G<G^{\prime}$ if and only if $W(G)<W\left(G^{\prime}\right)$. For an arbitrary string $w$ necessary and sufficient algebraic conditions are given for the existence of a graph $G$ such that $w \in\{w(G)\}$. (Received June 22,1956.)

\section{5t. R. L. Davis: Torsion in Engel modules.}

One standard approach to the Restricted Burnside Problem has been through the Engel ring defined by $\left[f g^{p-1}\right]=0$, over the field of $p$ elements. It was thus that A. L. Kostrikhin (Izvestia Akad. Nauk SSSR. vol. 19 (1955) pp. 233-244) just recently solved the problem affirmatively for the case $p=5$ and the number of generators $q=2$. This paper studies rather the free Lie $J$-module ( $J$ the integers) on two generators. If in this case the Engel ideal generated by elements $\left[f g^{4}\right]$ is replaced by an ideal $I$ generated by some of its "linear consequences," it can be proved that $L / I$ has torsion, which first appears in $L_{6}\left(L_{k}\right.$ is the submodule of Lie forms of degree $k$ ). It can then further be shown that actually $5 L_{7} \subset I$, and hence from this point on Kostrikhin's computations apply also to this $J$-module. (Received May 14,1956.)

\section{6t. M. M. Day: Amenability of semigroups. II.}

The concepts of amenability and strong amenability for semigroups and groups were defined and discussed in Trans. Amer. Math. Soc. vol. 69 (1950) pp. 276-291 and Bull. Amer. Math. Soc. Abstracts 55-11-506 and 56-1-40. It was shown then that strong amenability of a semigroup $\Sigma$ implied amenability of $\Sigma$; by finding new formulations of these properties of $\Sigma$ in terms of the semigroup algebra $l_{1}(\Sigma)$, it is proved here that the converse is also valid. (Received June 29, 1956.) 


\section{M. M. Day: The second conjugate of a semigroup algebra.}

Arens (Proc. Amer. Math. Soc. vol. 2 (1951) pp. 839-848) gave an example of a commutative Banach algebra whose second conjugate algebra is not commutative. This note shows that this is the natural situation for the semigroup algebra $l_{1}(\Sigma)$ of a semigroup $\Sigma$. In particular, if $\Sigma$ is the set of positive integers, there exists more than one invariant mean on $m(\Sigma)$ and if two such means are multiplied together the product is always the second one, so the subsemigroup of invariant means in the algebra $l_{1}(\Sigma) * *$ is not commutative. (Received June 29, 1956.)

\section{R. P. Dilworth: Homomorphisms of distributive lattices.}

The following theorem is proved: Let $M$ be a sublattice of a distributive lattice $L$. Let $\theta \geqq \phi$ be congruence relations on $M$ and let $\phi^{*}$ be an extension of $\phi$ to $L$. Then there exists an extension $\phi^{*}$ of $\theta$ to $L$ such that $\theta^{*} \geqq \phi^{*}$. This theorem is used to prove the following results: (1) A lattice is distributive if and only if every congruence relation on each sublattice can be extended to a congruence relation on the entire lattice. (2) If $M$ is a sublattice of a distributive lattice $L$, then the lattice of congruence relations on $M$ is a meet homomorphic image of the lattice of congruence relations on $L$. (3) If $M$ is a sublattice of a distributive lattice $L$, then the lattice of congruence relations on $M$ is a homomorphic image of the lattice of congruence relations on $L$ if and only if $M$ is projectively dense in $L$. (Received July 2, 1956.)

609. N. J. Divinsky. D-regularity.

An element $x$ of a ring $A$ is r.D.r. (right $D$-regular), if there exists an element $y$ in $A$ such that $x=x y$. An ideal is r.D.r. if every element in it is r.D.r. In every ring there exists a maximal r.D.r. ideal $M_{r}$ and a maximal 1.D.r. (left $D$-regular) ideal $M_{l}$ which are in general different from one another. The right subradical is the Jacobson radical of $M_{r}$. When $A$ has either DCC on right ideals or ACC on left ideals, $M_{r}=A$ if and only if $A$ has a right unity. Also $M_{r}=M_{l}=A$ if and only if $A$ has a unity when $A$ has any one of the following: DCC on one-sided ideals; ACC on one-sided ideals; a unity in $A / J$ where $J$ is the Jacobson radical; $A$ has a nondivisor of zero. If $A$ has DCC on one-sided ideals then $A$ is nilpotent if and only if $M_{r}=M_{l}=0$ and there are no nonzero total divisors of zero in $A^{n}$ for every positive integer $n$. For commutative rings with $M\left(=M_{r}=M_{l}\right)=0$ it is shown that when $A$ has DCC, $A$ is nilpotent; when $A$ has ACC, $A$ is a subdirect sum of nilpotent rings (though it may be Jacobson semisimple!); and in general $A$ is a subdirect sum of rings bound to their maximal nilideals. (Received June 19, 1956.)

\section{D. O. Ellis: Notes on the foundations of lattice theory, III.}

Various propositions are derived relating $m$-lattices (Garrett Birkhoff, Lattice Theory, American Math. Soc., New York, 1948), delta-lattices (Randall Conkling and David Ellis, Metric delta-lattices, Revista (Tucuman), vol. 10 (1954) pp. 75-82), c-groupoids (David Ellis, Notes on the foundations of lattice theory II, Archiv der Math. vol. 4 (1953) pp. 257-260), partially ordered and lattice ordered groupoids, and lattice properties in a given lattice. The methods are straightforward in general and are algebraic and lattice-theoretic in nature. (Received June 25, 1956.)

611. C. M. Fulton and D. A. Norton (p): A fixed point theorem for affine transformations.

Let the affine transformation $\sigma$ on an $n$-dimensional hyperspace be defined by 
$X^{\prime}=\sigma X=A X+C$ (where $A$ is an $n \times n$ square matrix and $C$ is a column vector). Define inductively $X^{(i)}=\sigma X^{(i-1)}$. If the hypervolume $V_{A}(X)=\mid X^{\prime}-X, X^{\prime \prime}-X, \cdots$, $X^{(n)}-X \mid$ is a nonzero constant, $\sigma$ can have no fixed point. This paper shows that a necessary and sufficient condition that $V_{A}(X)$ be a nonzero constant is that the minimal equation of $A$ be $(X-1)^{n}=0$ and that $C$ not be the null space of $A^{n-1}$. (Received May $28,1956$.

\section{2t. Lawrence Goldman: Algebraic results on homogeneous linear} differential equations.

Let $K$ be any differential field and let $L(y) \in K\{y\}$ be a homogeneous linear differential polynomial of order $n$. Let $K\left\langle\omega_{1}, \cdots, \omega_{n}\right\rangle$ be a Picard-Vessiot extension of $K$ (E. R. Kolchin, Ann. of Math., vol. 49, pp. 1-42) where $\left(\omega_{1}, \cdots, \omega_{n}\right)$ is a fundamental system of zeros of $L(y)$. Let $F(y) \in K\{Y\}$ be the lowest order irreducible differential polynomial such that $F\left(\omega_{1}\right)=0$, let the order of $F(y)$ be $r$. The following results are proved: (1) Over the differential field $K\left\langle\omega_{1}\right\rangle, L(y)$ factors (iteratively) into $L_{1} L_{2}(y)$ where $L_{1}, L_{2}$ are of orders $n-r, r$ respectively. (2) If $F$ is neither linear nor homogeneous then dimension of $K\left\langle\omega_{1}, \cdots, \omega_{n}\right\rangle$ over $K$ is $(n-1) r$, if $F$ is not linear but is homogeneous then dimension of $K\left\langle\omega_{1}, \cdots, \omega_{n}\right\rangle$ over $K$ is $\leqq(n-1) r+1$. (3) If $r=n-1$ and degree of $F$ is $m$ write $F=\sum_{0}^{m} F_{i}$, where $F_{i}$ is homogeneous of degree $i$, then the general manifold (J. F. Ritt, Differential algebra) of $\sum_{0}^{m} c_{i} F_{i}\left(c_{i}\right.$ any constants) belongs to the manifold of $L(y)$. (4) Let the $n \times n$ algebraic matric group of all automorphisms of $K\left\langle\omega_{1}, \cdots, \omega_{n}\right\rangle$ over $K$ be the orthogonal group then $F$ is of order $n-1, F=F_{m}+F_{0}$ and the lowest order irreducible differential equation over $K$ which any zero of $L(y)$ satisfies is of the form $F_{n}+c F_{0}=0$, where $c$ is some constant. (Received June 26,1956 .)

613. Franklin Haimo: An order preserving map on the subgroup lattice of the automorphism group.

Let $H$ be a group of automorphisms of a group $\alpha$, and let $\lambda(H)$ be the set of all automorphisms of $\alpha$ such that to each pair $a \in \alpha$ and $\bar{h} \in \lambda(H)$ there exists $k_{(a, \bar{h})} \in H$ with $k_{(a, \bar{h})} \bar{h}(a)=a$. Then $\lambda(H)$ is a subgroup of automorphisms, $\lambda(H) \supset H$, and $\lambda$ is monotonic on the lattice of subgroups of the automorphism group, carrying normal subgroups onto normal subgroups. $H$ and $\lambda(H)$ share their sets of universal fixed points in $\alpha$. If the elements of $H$ are precisely those automorphisms of $\alpha$ which induce the identity on some fixed quotient $\alpha / \beta$, then $\lambda(H)=H$. In any case $\lambda, \lambda(H)=\lambda(H)$. If $H$ is the group $J$ of inner automorphisms of $\alpha$, then $\lambda(H)$ consists of all the class preserving automorphisms of $\alpha$. In 1913 Miller showed that $\lambda(J)$ may include $J$ strictly, a result frequently neglected. [See G. E. Wall, J. London Math. Soc. vol. 22 (1947) pp. 315-320.] In any event, $\lambda(J)$ is part of the second centralizer of $J$. (Received July $5,1956$.

614. William Hanf (p) and Alfred Tarski: New results concerning isomorphism of Boolean algebras and commutative semigroups. Preliminary report.

The results below supplement and partly improve those in Bull. Amer. Math. Soc. Abstracts 60-6-651 and 60-6-668. The following two theorems have been established by Hanf: (I) There are denumerable Boolean algebras $\mathfrak{A}$ and $\mathfrak{B}$ such that $\mathfrak{A}$ and $\mathfrak{A} \times \mathfrak{B} \times \mathfrak{B}$ are isomorphic while $\mathfrak{A}$ and $\mathfrak{A} \times \mathfrak{B}$ are not. This result was obtained, following Tarski's suggestion, by modifying the construction of Kinoshita in Fund. Math. vol. 40, pp. 
$39-41$; it improves Kinoshita's result. (II) There is a (nondenumerable) Boolean algebra $\mathfrak{A}$ such that $\mathfrak{A}$ and $\mathfrak{A} \times \mathfrak{A} \times \mathfrak{A}$ are isomorphic while $\mathfrak{A}$ and $\mathfrak{A} \times \mathfrak{A}$ are not. Here for $\mathfrak{A}$ the algebra $\mathfrak{A} \times \mathfrak{B}_{1}$ of Abstract $60-6-651$ can be taken. From (I) Tarski has derived the following corollary: (III) There are denumerable Boolean algebras $\mathcal{Q}$ and $\mathbb{C}$ such that $\mathfrak{A} \times \mathfrak{A}$ and $\mathfrak{E} \times \mathfrak{C}$ are isomorphic while $\mathfrak{A}$ and $\mathfrak{C}$ are not. This solves Problem (1) in Abstract 60-6-668. Moreover, Tarski has pointed out that (I), (II), and (III) can be carried over to commutative semigroups. The problem of extending (II) to denumerable Boolean algebras and denumerable commutative semigroups remains open. (Received July 5, 1956.)

\section{5t. I. Heller: On sets of generators in a free Abelian group.}

If $G$ is a free Abelian group of rank $n$, a subset $S$ of $G$ containing at least $n$ linearly independent elements is called a set of generators when every set of $n$ linearly independent elements of $S$ is a basis of $G$. Using a result on matrices (the author, On linear systems with integral valued solutions, Bull. Amer. Math. Soc. Abstract 62-6-743 and 62-6-744) the note proves: If a set of generators contains $n(n+1)$ elements (not counting the null element), it is maximal and of the form $D=\left\{a_{i}, a_{i}-a_{i}\right\}$, where the $a_{i}$ are a basis of $G$. For $n \geqq 4$ there are maximal sets of generators with less than $n(n+1)$ elements (and hence not of form $D$ ). (Received June 4, 1956.)

\section{6t. J. H. Hodges: Weight partitions for skew matrices over a finite} field.

For $\alpha \in G F(q), q=p^{n}, p$ odd, let $e(\alpha)=e^{2 \pi i t(\alpha) / p}$, where $t(\alpha)=\alpha+\alpha^{p}+\cdots+\alpha^{p^{n-1}}$. If $A=\left(\alpha_{i j}\right)$ is a square matrix with elements in $G F(q)$, let $\sigma(A)=\sum_{i} \alpha_{i i}$. Consider the sum $S=S(B, U, A)=\sum e\left\{\sigma\left(U^{\prime} X+X^{\prime} U\right)\right\}$, where $A$ is a nonsingular skew matrix of order $2 m, B$ is a skew matrix of order $t, U$ is an arbitrary $2 m \times t$ matrix, and the summation is over all $2 m \times t$ matrices $X$ satisfying the equation $X^{\prime} A X=B$, all matrices having elements in $G F(q)$. If $U=0, S$ is the number $Z_{t}(A, B)$ of solutions $X$ of $X^{\prime} A X$ $=B$, which is given in (L. Carlitz, Representations by skew forms in a finite field, Archiv der Mathematik, vol. 5 (1954) pp. 19-31). In the present paper it is shown that $S$ can be expressed in terms of generalized Kloosterman sums defined for skew matrices over $G F(q)$. A number of properties of these Kloosterman sums are also given. The analogous problems for symmetric and for general matrices have been considered previously by the author and will appear presently in Mathematische Zeitschrift and the Duke Mathematical Journal, respectively. The symmetric case with $t=1$ has been considered with more explicit results by Carlitz in (L. Carlitz, Weighted quadratic partitions over a finite field, Canadian Journal of Mathematics vol. 5 (1953) pp. 317323). (Received July 5, 1956.)

617t. D. R. Hughes: Collineations and generalized incidence matrices. Preliminary report.

Let $\pi$ be a $(v, k, \lambda)$ configuration, let $G$ be a collineation group of $\pi$, where $G$ has order $m$. Let $w$ be the number of transitive classes of points of $\pi$, with respect to $G$; then $w$ is also the number of transitive classes of lines. There exist integers $r_{i}, s_{i}$, $i=1,2, \cdots, w$, where each $r_{i}$ and $s_{i}$ divides $m$, such that if $C_{1}=\operatorname{diag}\left(s_{1}^{-1}, s_{2}^{-1}, \cdots\right.$, $\left.s_{w}^{-1}\right), C_{2}=\operatorname{diag}\left(r_{1}^{-1}, r_{2}^{-1}, \cdots, r_{w}^{-1}\right)$, and if $S$ is the square matrix of order $w$ with +1 in every position, then $A C_{1} A^{T^{w}}=(k-\lambda) C_{2}^{-1}+\lambda m S, A^{T} C_{2} A=(k-\lambda) C_{1}^{-1}+\lambda m S$, where $A$ is a square matrix with non-negative integral entries. The Hasse-Minkowski theory of rational congruence can be applied to these matrix equations: for example, if $m$ 
is an odd prime and if the number $N$ of points fixed by a nonidentity element of $G$ is even, then $x^{2}=(k-\lambda) y^{2}+(-1)^{\epsilon} m z^{2}, \epsilon=(m-1) / 2$, possesses a nontrivial solution in integers. Applications of these results to projective planes (i.e., $\lambda=1$ ) are numerous, yielding many restrictions on the structure of possible planes of non-prime-power order. (Received July 11, 1956.)

\section{Burrowes Hunt: Continued fractions and indefinite forms.}

There is given an explicit matric formulation of the connection between indefinite binary forms and regular continued fractions; this is extendable to $n$-ary forms and a continued-fraction-like algorithm. Let $A$ and $B$ be the matrices with top row $(1,1)$ and bottom rows $(0,1)$ and $(1,0)$. If $a_{0}$ is an integer and $a_{1}, a_{2}, \ldots$ are non-negative integers, then $F=\cdots B A^{a_{2}} B A^{a_{1}} B A^{a_{0}} B$ is called a Farey Matrix. Let the rows of $F$ be $(a, b)$ and $(c, d): c / d$ is equal to the r.c.f. $\left[a_{0}+1 ; a_{1}+1, a_{2}+1, \cdots\right]$. To $F$ is associated the form $b x^{2}+(a-d) x y-c y^{2}$ of discriminant trace ${ }^{2} F-4 \operatorname{det} F$. Cyclic permutation of the letters spelling $F$ gives rise to a chain of equivalent reduced forms. If $F^{\prime}$ has the same trace and determinant as $F$ but is not a cyclic permutation of $F$, then $F$ and $F^{\prime}$ are in different classes. If forms are associated to all $2 \times 2$ matrices in this way then $M$ is equivalent to $N$ iff $M=U^{-1} N U$ for a unimodular $U$. Every unimodular $U$ has a unique representation $U=G F$, where $F$ is a Farey Matrix and $G$ is one of a group of 8 matrices whose effect on $F$ is to permute the rows or multiply either or both rows by -1 . To each class of indefinite forms is associated the equivalent (to each other) Farey Matrices which are automorphs of appropriate forms in the class. (Received July 5, 1956.)

619. W. E. Jenner: On representations of the full linear group and some of its subgroups over infinite fields of prime characteristic.

It is shown that if $k$ is an infinite field of characteristic $p>0$ then for $n>1$ the groups $G L(n, k), S L(n, k)$ and $S p(2 n, k)$ have rational irreducible representations of arbitrarily high degree. For the first two groups this had been shown by M. Rosenlicht (unpublished). This result is in contrast to the fact that the degrees of the absolutely irreducible representations of any finite-dimensional Lie algebra over a field of characteristic $p>0$ are bounded (Jacobson, Zassenhaus). Preliminary results are obtained towards a complete representation theory for these groups. (Received July 12, 1956.)

620t. Bjarni Jónsson: Results on direct decompositions of groups and other algebraic systems.

Theorem. Given an infinite Boolean algebra $\mathfrak{A}$ and a denumerable, centerless, indecomposable group $\mathbb{( E ,}$, there exists a centerless group $(\mathbb{S}$ with the same cardinal as $\mathfrak{A}$, and an isomorphism $F$ of $\mathfrak{A}$ onto the (Boolean) algebra of factors of $\mathbb{B}$, such that $F(a) \cong F(b)$

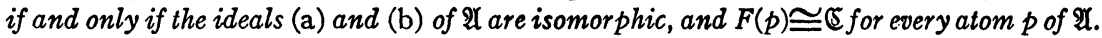
Combined with results by Hanf and Tarski (Bull. Amer. Math. Soc. Abstracts 60-6651, 60-6-668 and 62-6-614), this yields (I) a (nondenumerable) group $\&$ and a finite group $\mathfrak{S}$ such that $\mathfrak{S} \cong(\mathfrak{S} \times \mathfrak{S} \times \mathfrak{S}$ but not $\mathfrak{S} \cong(\mathfrak{S} \times \mathfrak{S}$, (II) denumerable groups $\mathbb{E}$ and $\mathfrak{S}$ such that $\mathfrak{S} \cong(\mathfrak{S} \times \mathfrak{S} \times \mathfrak{S}$ but not $\mathfrak{S} \cong(\mathfrak{S} \times \mathfrak{S}$, (III) a (nondenumerable) group

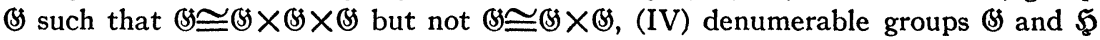
such that $\mathbb{S} \times(\mathfrak{S} \cong \mathfrak{S} \times \mathfrak{S}$ but not $\mathfrak{S} \cong \mathfrak{S}$. Actually, for "group" one may substitute " $K$-algebra," $\boldsymbol{K}$ being any class of algebras with zero and finitary operations, closed under the operation of taking subdirect powers and containing a denumerable, center- 
less, indecomposable algebra $\mathfrak{C}$, except that for (I) $\mathfrak{C}$ must be assumed finite. (For terminology see J6nsson and Tarski, Direct decompositions of finite algebraic systems.) Thus generalized, (I)-(IV) include the results by Hanf and Tarski. (Received July 5, 1956).

\section{1t. Bjarni Jónsson and Alfred Tarski: Two general theorems con-} cerning free algebras.

Given a class $\boldsymbol{K}$ of algebraic systems, an algebra $\mathfrak{X} \in \boldsymbol{K}$ is called a $\boldsymbol{K}$-algebra freely generated by a set $G$ of its elements if $\mathscr{A}$ is generated by $G$ and every mapping of $G$ into an algebra $\mathfrak{B} \in K$ extends to a homomorphism of $\mathfrak{A}$ into $\mathfrak{B}$. Many familiar classes $\boldsymbol{K}$ (groups, rings, lattices) satisfy the conditions: $\left(\mathrm{C}_{1}\right)$ a $\boldsymbol{K}$-algebra $\mathfrak{A}$ freely generated by a finite set with $n$ elements is never generated (freely or not) by a set with $p<n$ elements; $\left(\mathrm{C}_{2}\right)$ if, moreover, $\mathfrak{A}$ is generated by another set $H$ with $n$ elements, it is freely generated by $H$. Theorems: (I) Every class $\mathbf{K}$ containing a finite algebra with more than one element satisfies $\left(\mathrm{C}_{1}\right)$. (II) If every equation holding in all finite $K$-algebras holds identically in all $\boldsymbol{K}$-algebras, then $\mathrm{K}$ satisfies $\left(\mathrm{C}_{2}\right)$. In connection with (I) compare Fujiwara, Proc. Jap. Acad. 31, p. 135. $\left(\mathrm{C}_{1}\right)$ fails in the class $K_{1}$ of algebras (with operations $0,{ }^{+},{ }^{*}$ ) characterized by the axioms $x^{+} \circ x^{*}=x,(x \circ y)^{+}=x,(x \circ y)^{*}=y$; in fact, any two $\boldsymbol{K}_{1}$-algebras freely generated by finite sets are isomorphic. The class $\boldsymbol{K}_{2}$ characterized by the single axiom $x^{+*}=x$ satisfies the hypothesis of (I) and hence condition $\left(\mathrm{C}_{1}\right)$; but $\left(\mathrm{C}_{2}\right)$ fails in $K_{2}$ even for $n=1$. (Received July 5,1956 .)

\section{2t. C. W. Kohls: Ideals in rings of continuous functions. I.}

Let $C(X)$ be the ring of all continuous real functions on a completely regular Hausdorff space $X ; N^{p}=\{f \in C(X): f=0$ on some $X$-neighborhood of $p\}$; and $M^{\prime p}$ $=\{f \in C(X)$ :the zero-set of $f$ meets every deleted nbhd. of $p\}$, if $p$ is a nonisolated point of $X,=M^{p}$ otherwise. For any $p \in \beta X, p$ is a $\beta F$-point (with respect to $X$ ) if whenever $f \in C(X)$ is extendable to $p$ and vanishes there, then $f \geqq 0$ or $f \leqq 0$ on some $X$-nbhd. of $p$. (1) $p$ is a $\beta F$-point iff $N^{p}$ is prime, and iff the zero-sets of any pair of functions extendable to $p$ and vanishing there are comparable in some $X$-nbhd. of $p$. (2) Let $p$ be a $\beta F$-point. Then $C(X) / N^{p}$ and $C(X) / M^{\prime} p$ are ordered integral domains containing the reals $R$, which contain infinitely large elements iff $p \notin v X$ (largest subspace of $\beta X$ over which all $f \in C(X)$ can be extended). If $p \notin v X$, then $C(X) / N^{p}$ is an $\eta_{1}$-set, but if $p \in v X, C(X) / N^{p}$ has a countable cofinal subset. The field of quotients of $C(X) / N^{p}$ is a real-closed field. $C(X) / M^{\prime p}$ is a valuation ring, and hence the prime ideals containing $M^{\prime} p$ form a chain. (3) On the other hand, if $p$ is not a $\beta F$-point, there are incomparable prime ideals contained in $M^{p}$, and hence $C(X) / N^{p}$ is not a valuation ring. (The author was supported by the National Science Foundation.) (Received April 10, 1956.)

\section{3t. C. W. Kohls: Ideals in rings of continuous functions. II.}

Let $C^{*}(X)$ be the ring of bounded continuous real functions on a locally compact Hausdorff space $X ; C_{\infty}(X)$, the functions "vanishing at $\infty$ "; $C_{s}(X)$, the functions with compact supports. An ideal $A$ of an arbitrary ring $B$ is a 3 -ideal if for $b \in B$, whenever there exists $a \in A$ such that $a \in M$ iff $b \in M$, for all $M \in \mathfrak{M}_{B}(B)$ (=structure space of $B$ ), then $b \in A$. (1) If $A$ is an ideal of $C^{*}(X)$, then (a)-(c) are equivalent: (a) $C_{s}(X)$ $\subseteq A \subseteq C_{\infty}(X)$; (b) for all $M \in \mathfrak{M}_{\delta}\left(C^{*}(X)\right), M \supseteq A$ iff $M$ is a free ideal; (c) the mapping $p \rightarrow M^{* p} \cap A(p \in X)$ is a homeomorphism from $X$ to $\mathfrak{M}_{s}(A)$. (2) $C_{s}(X)$ is an ideal of $C_{\infty}(X)$ contained in no maximal ideal. (3) $C_{\infty}(X)$ is a regular ring iff $X$ is finite. Using 
a result of L. J. Heider (Bull. Amer. Math. Soc. Abstract 62-1-158), the set of 3-ideals $A$ of $C^{*}(X)$ satisfying $C_{s}(X) \subseteq A \subseteq C_{\infty}(X)$ is characterized algebraically. For $C_{s}(X)$, the conditions become: (i) $A$ is a commutative semi-simple algebra over $R$ such that $M \in \mathfrak{M}_{s}(A)$ implies $A / M \cong R$; (ii) each $a \in A$ has a relative identity, i.e., there exists an $e \in A$ such that $a e=a$; (iii) for each $a \in A$, there is an $r \in R$ such that $a \leqq r e$, where $e$ is a suitable relative identity for $a$ and $\leqq$ is the induced partial order on $A$; (iv) if $B$ is an extension of $A$ satisfying (i)-(iii) and such that the mapping $M \rightarrow M \cap A$ is one-to-one from $\mathfrak{M}_{s}(B)$ onto $\mathfrak{M}_{s}(A)$, then $B=A$. (The author was supported by the National Science Foundation.) (Received April 10, 1956.)

\section{4t. C. W. Kohls: The space of prime ideals of a ring.}

Let $\mathfrak{S}(A)$ be any set of ideals of the ring $A$ which admits the Stone topology; $\mathfrak{P}(A)$, the set of primitive ideals; and $\mathfrak{Q}(A)$, the set of prime ideals-endowing each set with the Stone topology. For commutative $A, e \in A$ is a relative identity for $a \in A$ if $a e=a$. (1) Let $A$ be a ring in which every proper ideal is contained in an S-ideal. Then $\subseteq(A)$ is compact iff $A$ is finitely generated. (2) The space $\mathfrak{S}(A) \subseteq \mathfrak{Q}(A)$ is Hausdorff iff for each distinct pair $S, T \in \subseteq(A)$, there exist $a, b \in A$ such that $a \notin S$, $b \notin T$ and $a x b \in \Delta S(A)$ (=intersection of all $S \in \subseteq(A)$ ) for all $x \in A$. (3) Suppose $A$ is commutative, $\mathfrak{Q}(A) \supseteq \mathfrak{S}(A) \supseteq \mathfrak{P}(A)$, and for each $S \in \mathfrak{S}(A)$, there is an $a \notin S$ with a relative identity. Then $\mathfrak{S}(A)$ is locally compact. And if $\Delta \subseteq(A)=(0)$, then $\mathfrak{S}(A)$ is compact iff $A$ has an identity. (4) Let $I$ be a proper ideal of $A, P \in \mathfrak{Q}(I)$, and $Q$ $=\{a \in A: I a \subseteq P\}$. Then $Q \in \mathfrak{Q}(A)$, and $P=Q \cap I$. (5) If $I$ is a proper ideal of $A$, $\{Q \in \mathfrak{Q}(A): Q \nsupseteq I\}$ is homeomorphic to $\mathfrak{Q}(I)$, under the mapping $Q \rightarrow Q \cap I$. (6) Suppose $A$ is commutative with characteristic $n \neq 0, \mathfrak{Q}(A)(\mathfrak{P}(A))$ is Hausdorff, and for each $P \in \mathfrak{Q}(A)(\mathfrak{P}(A))$ there is an $a \notin P$ with a relative identity. Then $\mathfrak{Q}\left(A ; I_{n}\right)$ - $\left(\mathfrak{B}\left(A ; I_{n}\right)\right)$ is Hausdorff, where $\left(A ; I_{n}\right)$ is the ring consisting of pairs $(a, i), a \in A$, $i \in$ integers $\bmod (n)$, with operations defined so that $(0,1)$ is the identity. (The author was supported by the National Science Foundation.) (Received June 4, 1956.)

\section{L. A. Kokoris: On algebras of $(\gamma, \delta)$ type.}

An algebra of $(\gamma, \delta)$ type is a finite dimensional algebra $A$ over a field $F$ satisfying the identities $z(x y)=(z x) y+\gamma(x z) y-\gamma x(z y)+\delta(y z) x-\delta y(z x)$ and $(x y) z=x(y z)+\gamma(x z) y$ $-\gamma x(z y)+(\delta-1)(y z) x-(\delta-1) y(z x)$ where $\gamma$ and $\delta$ are elements in $F$ such that $\gamma^{2}-\delta^{2}+\delta$ $=1$. The structure of algebras with $\delta \neq 0,1$ has been studied previously. (See A. A. Albert, Almost alternative algebras, Portugaliae Mathematica, vol 8 (1949) pp. 2336 , and the author's, On a class of almost alternative algebras, Canadian Journal of Math. vol. 8 (1956) pp. 250-255.) Algebras of type $(\gamma, \delta)=(-1,0)$ and $(1,1)$ are studied here. The results for simple and semisimple algebras are the same as when $\delta \neq 0,1$, but the proofs differ due to the fact that the decompositions relative to an idempotent have different properties in these two cases. (Received June 4, 1956.)

626t. Joseph Landin and Irving Reiner: Automorphisms of the general linear group over a principal ideal domain.

Let $R$ be an integral domain, possibly noncommutative, in which all left and right ideals are principal. Let $E$ be a free $R$-module of rank $n$, and $E^{*}$ its dual. Let $\Gamma L_{n}(R)$ be the group of invertible semi-linear maps of $E$ onto itself, relative to automorphisms of $R$, and let $G L_{n}(R)$ be its subgroup of linear maps. Define a subgroup $U$ of $G L_{n}(R)$ by $U=\left\{\lambda_{\alpha}: \alpha=\right.$ unit in the center of $\left.R\right\}$, where $\lambda_{\alpha}$ is the homothetic map $x \rightarrow x \alpha$. If $\phi: G L_{n}(R) \rightarrow U$ is any homomorphism such that $\lambda_{\alpha} \in U, \phi\left(\lambda_{\alpha}\right)=\lambda_{\alpha}^{-1}$ implies $\alpha=1$, and 
if $g \in \Gamma L_{n}(R)$, then the mapping $u \rightarrow \phi(u) g u g^{-1}$ gives an automorphism of $G L_{n}(R)$. Likewise, so does $u \rightarrow \phi(u) h^{-1} u^{*} h$, where $h$ is a one-to-one semi-linear map of $E$ onto $E^{*}$ relative to an anti-automorphism of $R$, and where $u^{*}$ is the contragredient of $u$. It is shown here that, conversely, every automorphism of $G L_{n}(R)$ for $n \geqq 3$ is given by one of these formulas. The proof uses methods of Mackey (Ann. of Math. vol. 43 (1942) pp. 244-260), Rickart (Amer. J. Math. vol. 72 (1950) pp. 451-464), Dieudonné (La géométrie des groupes classiques, 1955), and Reiner (Trans. Amer. Math. Soc. vol. 79 (1955) vol. 459-476). (Received April 30, 1956.)

\section{W. J. LeVeque: On the frequency of small fractional parts in certain real sequences.}

Let $x$ be a number in $[0,1]$, and let $X_{n}=\langle n x\rangle$, the distance between $n x$ and the nearest integer to $n x$. Let $f(x)$ be a suitable positive decreasing function with $\sum_{1}^{\infty} f(k)$ $=\infty$, and put $g(x)=f(\log x) / x, A(n)=\sum_{1}^{n} g(k)$. Let $T_{n}(x)$ be the number of positive integers $m \leqq n$ such that $X_{m}<g(m)$. It is shown that for fixed $\omega, \lim _{n \rightarrow \infty}$ meas $\left\{x \mid T_{n}(x)\right.$ $\left.<12 \pi^{-2} A(n)+\omega\left(12 \pi^{-2} A(n)\right)^{1 / 2}\right\}=\phi(\omega)$, where $\phi(\omega)$ is the normal distribution function $(2 \pi)^{-1} \int_{-\infty}^{\omega} e^{-t^{2} / 2} d t$. This refines a well-known theorem of Khinchin, which guarantees the existence of infinitely many solutions of the inequality $X_{m}<g(m)$. The result reflects the strong statistical dependence of the quantities $\langle m x\rangle$, since if the $X_{n}$ were redefined as independent random variables uniformly distributed on $[-1 / 2,1 / 2]$, the same theorem would hold with the factor $12 \pi^{-2}$ replaced by 2 . The same problem is considered for the case that $X_{n}=\left(r_{1} r_{2} \cdots r_{n} x\right)$, where $\left\{r_{i}\right\}$ is a suitable increasing sequence of positive integers, and here the theorem to be expected from the independent case again holds. Moreover, it is shown in this case that for almost all $x$, $T_{n}(x) \sim 2 \sum_{1}^{n} g(k)$. (Received July 6, 1956.)

\section{D. J. Lewis: Strongly normic polynomials.}

To each homogeneous polynomial $F\left(x_{1}, x_{2}, \cdots, x_{n}\right)=F(X)$ over the ring of integers $\mathcal{\theta}$ of a $\rho$-adic field such that $F(X)$ has only the trivial zero in $\theta$, there exists a smallest rational integer $m$ such that $F\left(a_{1}, a_{2}, \cdots, a_{n}\right) \equiv 0\left(\bmod \rho^{m}\right), a_{i}$ in $\mathcal{O}$, only if every $a_{i} \equiv 0(\bmod \rho)$. (Here $\rho$ is the prime ideal in $\mathcal{O}$.) If $m$ is equal to the degree of $F(X)$, then $F(X)$ is said to be a strongly normic polynomial over $\mathcal{O}$. It is shown that there exists polynomials $\phi_{d}(y)$ of degree $d-1$ over the rational integers such that if $F(X)$ is a strongly normic polynomial over $\mathcal{O}$ of degree $d$, and if $d$ is less than the number $q$ of elements in $\Theta / \rho$; then $n<d^{2} \phi_{d}(q-1)$. The proof makes use of certain results concerning ideals in rings of polynomials. (See Amer. J. Math. vol. 78 (1956) pp. 71-77.) (Received July 2, 1956.)

\section{9t. Saunders MacLane: The categorical bar construction and the} homology of rings.

For a ring $\Lambda$ there is a natural complex $R(\Lambda)$ of $\Lambda$-bimodules giving the homology of $\Lambda$, with 2-dimensional cohomology the group of all ring extensions by $\Lambda$ with a nilring as kernel. $R$ is formed by a modification of the bar construction $B(Q, \eta)$, with $Q$ a graded differential ring, $\eta: Q \rightarrow \Lambda$ a homomorphism of such. This construction, described by means of abelian categories, provides a universal pattern for all standard constructions of homological algebra. Take $\mathfrak{K}$ the category of graded differential right $\Lambda$-modules $\mathcal{K}, \mathfrak{L}$ the subcategory of $Q-\Lambda$-bimodules, and $T: \mathcal{K} \rightarrow \mathfrak{L}$ the universal functor $T(K)=Q \otimes K$ with the immersion $\tau(k)=1 \otimes k$ of degree +1 . Set $B_{n}$ $=T^{n+1}(\Lambda)$. There is a given differential $\partial_{r}$ in $B_{n}$, and in addition a differential $\partial_{s}: B_{n}$ 
$\rightarrow B_{n-1}$ with $\tau$ as its contracting homotopy. Then $\partial_{s} \partial_{r}=-\partial_{r} \partial_{s}$ and $B$ is also acyclic for the differential $\partial_{r}+\partial_{s}$. In the quotient $\bar{B}=\Lambda \otimes_{Q} B$ the $\partial_{r}$ and $\partial_{s}$ are given by the formulas of Eilenberg-MacLane (Ann. of Math. vol. 58 (1953) p. 73) with $G$ replaced by $Q, J$ by $\Lambda$. The cubical complex $Q(\Lambda)$ of the additive group of $\Lambda$ with augmentation $\eta$ to $\Lambda$ becomes a graded differential ring with a suitable product. One then defines $R(\Lambda)=\bar{B}(Q(\Lambda), \eta)$. (Received June $26,1956$.

\section{0t. Saunders MacLane: Relative homology for abelian groups and} modules.

Relative homology theory, known to be intractable for groups, can be managed for abelian groups $A$ in terms of the cubical complex $Q(A)$. (cf. Eilenberg-MacLane, Trans. Amer. Math. Soc. vol. 71 (1951) pp. 294-330). In fact, for an exact sequence $0 \rightarrow A \rightarrow E \rightarrow C \rightarrow 0$ of abelian groups one proves that the induced map $Q(E) / Q(A)$ $\rightarrow Q(C)$ is a chain equivalence. The requisite reverse map and the homotopy are constructed by a method of successive approximations due to Eilenberg-Mac Lane. Suppose next that $\Lambda$ is a ring and $A, E$, and $C$, are $\Lambda$-modules. Using the categorical bar construction $B$ (see Abstract 629 above) one may then construct a natural complex $M_{\Delta}(C)=Q(C) \otimes_{Q(\Lambda)} B(Q(\Lambda), \eta)$ for each $\Lambda$-module $C$. By the same type of successive approximation, the induced map $M(E) / M(A) \rightarrow M(C)$ is again a chain equivalence. It follows that $M_{\Lambda}(C)$ is a standard and functorial projective (in fact, free) resolution of $C$. Hence $M$ gives direct expressions for the functors Tor and Ext of Cartan-Eilenberg, and for the projective homotopy groups of Eckman-Hilton. (Received June 26, 1956.)

\section{1t. D. W. Miller: On uniform semigroups.}

A semigroup $S$ is termed (i) uniform if $x S \cup S x=y S \cup S y$, all $x, y \in S$;(ii) completely uniform if $x S \cup S x=S$, all $x \in S$; (iii) a left (right) semigroup if $x y=x(x y=y)$, all $x, y \in S$ (iv) a zero semigroup if $S^{2}$ has order 1 . The following structural results are obtained. A completely uniform semigroup $S$ is isomorphic to the direct product of a left (or right) semigroup and a group if and only if $S$ contains at least one idempotent. A uniform semigroup containing exactly one idempotent is isomorphic to a subdirect product of a zero semigroup and a group; conversely, if $Z$ is a zero semigroup and $G$ a group then every subdirect product of $Z$ and $G$ is a uniform semigroup with just one idempotent. The class of uniform semigroups containing more than one idempotent is characterized by means of a semigroup constructed from an arbitrary group, two arbitrary sets, and two single-valued mappings. A completely uniform semigroup without idempotents is shown to admit division on one side, and hence is imbeddable in a generalized Baer-Levi semigroup (Teissier, C. R. Acad. Sci. Paris vol. 236 (1953) pp. 1120-1122); if $S$ is a uniform semigroup without idempotents, then $S^{2}$ admits division on one side. (Received July $5,1956$. )

\section{C. N. Moore: Patterns and prime pairs.}

If we stop the process known as the sieve of Eratosthenes at the end of a finite number of steps we obtain a sequence of integers relatively prime to each of the primes used in forming the sieve. The integers in this sequence less than the square of the following prime are absolutely prime. The set of integers which are less than or equal to $P_{k}=2 \cdot 3 \cdot 5 \cdot \cdots \cdot p_{k}$, where $p_{k}$ is the last prime used, form a pattern for all following integers, since any following sequence of length $P_{k}$ can be found by adding a proper multiple of $P_{k}$ to the integers of the original sequence. Such a sequence, or its successive differences, form a pattern for the whole distribution. It is the purpose 
of the present discussion to develop certain theorems concerning these patterns and to apply them to problems relative to the distribution of primes, in particular to the problem of prime pairs. The pattern obtained by deleting 3 and its successive multiples contains all the prime pairs except the first one. As we delete succeeding primes and their multiples, we obtain what may be termed patterns of prime pairs. The relationship between successive patterns of this sort gives us information concerning the asymptotic behavior of the prime pair distribution. (Received July 3, 1956.)

633. T. S. Motzkin, K. E. Ralston, and J. L. Selfridge (p): Minimal overlapping under translation.

Let the integers of the interval $(1,4 n)$ be divided into any two equal disjoint classes $\left\{a_{i}\right\}$ and $\left\{b_{i}\right\}, i=1(1) 2 n$. Must there exist an integer $t$ such that $a_{i}+t=b_{j}$ has at least $n$ solutions $(i, j)$ ? (P. Erdös, Some remarks on number theory, Riveon Lematematika vol. 9 (1955) pp. 45-48.) A $k$-tuple $c_{1} c_{2} \cdots c_{k}$ shall mean: the first $r c_{1}$ integers are $a$ 's, the next $r c_{2}$ are $b$ 's, the next $r c_{3}$ are $a$ 's, etc., alternating, where $r=4 n / \sum c_{i}$. A negative answer to the above question is furnished by the quintuple 35123 , which has the property that there are $\leqq 6 \mathrm{~m} / 7$ solutions for any $t$; we write $\rho(35123)=6 / 7$. This is the smallest $\rho$ for any subdivision with $k<7$, but $\rho(5314155)=5 / 6$ (e.g. with $n=6$ ). After Selfridge found the above examples, he and Ralston coded SWAC to search for others. The smallest $\rho$ found was $4 / 5=\rho(4124111612214)$. P. Scherk has shown that $\rho>2-2^{1 / 2}$. (Received August 16, 1956.)

\section{4t. R. B. Reisel: A note on the Malcev theorem.}

Let $A$ be as associative algebra over the field $F$ having Jacobson radical $N$ such that $A / N$ is locally separable, $\cap_{k=1}^{\infty} N^{k}=\{0\}$, and $A$ is complete with respect to the topology in which the powers of $N$ form a fundamental system of neighborhoods of zero. Suppose that $A$ contains subalgebras $S$ and $S^{*}$ such that $A=S \oplus N=S^{*} \oplus N$ (vector space direct sums). This note is concerned with finding a condition which will insure that $S$ can be mapped onto $S^{*}$ by an inner automorphism of $A$ generated by an element of the form $(1-r), r$ in $N$. The condition obtained here is that the set $N$ be complete with respect to a topology defined as follows: for each finite dimensional separable subalgebra $E$ of $S$, let $N(E)$ be the set of elements $r$ in $N$ such that the inner automorphism of $A$ generated by $(1-r)$ is the identity on $E$; for each $s$ in $N$ a fundamental system of neighborhoods of $s$ is defined to be the collection of the sets $N(E) \circ s$, where $r \circ s=r+s-r s$, for $E$ running through all finite dimensional separable subalgebras of $S$. (Received June 29, 1956.)

\section{5t. Alex Rosenberg: On the structure of the infinite general linear} group.

Let $L$ be the ring of all linear transformations on a vector space of countably infinite dimension over a division ring. Let $F$ be the (unique) ideal of the elements of $L$ with finite dimensional range, $Z$ the center of $L$, and $G$ the group of invertible elements of $L$. Theorem: There is no proper normal subgroup of $G$ containing the group $H$ of elements of the form $z+f, z$ in $Z$ and $f$ in $F ;$ i.e. $G / H$ is simple. The main part of the proof consists in showing that $G$ is generated by its elements of order two, or equivalently by the elements of the form $1+T, T^{2}=0$. (Received June 29, 1956.)

636. H. J. Ryser: Combinatorial properties of 0,1 matrices.

Let $A$ be a 0,1 matrix of size $m$ by $n$, with $r_{i}$ denoting the sum of row $i$ and $s_{j}$ of 
column $j$. Define the row and column sum vectors of $A$ by $R=\left(r_{1}, \cdots, r_{m}\right)$ and $S=\left(s_{1}, \cdots, s_{n}\right)$, respectively. Let $\bar{A}$ with column sum vector $\bar{S}$ be a 0,1 matrix of size $m$ by $n$ such that the 1's in each row of $\bar{A}$ are shifted to the left as far as possible. Let $S$ be a vector with non-negative integral components and let $S$ be majorized by $\bar{S}$ (Hardy, Littlewood, Polya, Inequalities, Cambridge, 1952). Then by rearranging 1's in the rows of $\bar{A}$, one may construct a matrix $A$ having column sum vector $S$. An interchange is a transformation of four elements of $A$ which replaces a two by two identity submatrix of $A$ by $\left[\begin{array}{ll}0 & 1 \\ 1 & 0\end{array}\right]$ or vice versa. Let $A$ and $A^{*}$ be two $m$ by $n$ matrices composed of 0 's and 1's, possessing equal row sum vectors and equal column sum vectors. Then $A$ is transformable into $A^{*}$ by a finite number of interchanges. Applications to latin rectangles and to systems of distinct representatives are studied. (Received June $27,1956$.

\section{R. L. San Soucie: Weakly standard rings.}

Albert introduced the topic of standard rings, defined by (1) $(w x, y, z)+(x z, y, w)$ $+(w z, y, x)=0$ and $(2)(x, y, z)+(y, z, x)-(y, x, z)=0$, and showed that simple, finite dimensional algebras are standard if and only if they are either associative or Jordan algebras. Kleinfeld (Bull. Amer. Math. Soc. Abstract 62-1-127) remarked that (1) implies (3), $((w, x), y, z)=0$ and generalized Albert's result by proving that simple rings satisfy (2) and (3) if and only if they are either associative or commutative. Set $z=x$ in (2) and see that the generalized standard rings of Kleinfeld are flexible. In a flexible ring $R$, let $S=[s \in R \mid(s, x, y)=0]$ and let $M=[m \in R \mid(x, m, y)=0]$. Then (2) implies that $S \subset M$ and (3) states that $(x, y) \in S$. Call a ring $R$ weakly standard if $R$ is flexible and commutators are in $S \cap M$. The author proves that weakly standard rings are simple if and only if they are either associative or commutative, thus generalizing Kleinfeld's result. Finally, let $R$ be primitive if $R$ contains a maximal regular right ideal $A$ which contains no two sided ideal of $R$ other than the zero ideal. Then the author proves that a primitive weakly standard ring is either commutative or associative. (Received March 30, 1956.)

\section{A. Sklar: Dirichlet series and summation formulas.}

A class of meromorphic functions is considered, each member of which can be represented, in an appropriate half-plane, as an absolutely convergent Dirichlet series of the form $\sum_{n=1}^{\infty} a(n) \lambda_{n}^{-8}\left(0<\lambda_{1}<\lambda_{2}<\cdots\right)$. For every function of the class an identity involving sums of the form $\sum_{\lambda_{n} \leqq_{x}} a(n)\left(x-\lambda_{n}\right)^{q}(x>0, q$ complex) is obtained. The identities so obtained lead, in a quite simple fashion, to summation formulas which give expressions for sums of the form $\sum_{n=n}^{n_{2}} a(n) f\left(\lambda_{n}\right)$ where $f$ is any function satisfying certain not very stringent conditions. The results include the summation formulas of Poisson, Voronoi, Hardy-Landau and certain of those of Ferrar and Guinand as special cases and lend themselves to a variety of application in analytic number theory. (Received July 2, 1956.)

\section{9t. D. W. Wall: Sub-quasigroups of finite quasigroups.}

Lagrange's theorem for finite groups does not hold for finite quasigroups, i.e., the order of a sub-quasigroup need not divide the order of the quasigroup. However, certain relationships can be obtained between the order of the quasigroup and the orders of its sub-quasigroups. Let $Q$ be a quasigroup of order $n$. If $S$ is a sub-quasigroup of order $s$ then $n \geqq 2 s$. Let $R$ and $S$ be sub-quasigroups of order $r$ and $s$, respectively. If $R$ and $S$ intersect their intersection is a sub-quasigroup. If $R$ and $S$ intersect in a sub-quasigroup $P$ of order $p$, then the following can be proved: I. $n \geqq r+s+\max (r, s)$ 
$-2 p$. II. If $n=r+s+\max (r, s)-2 p$ then $r=s$ if and only if the subset $P \cup[Q \backslash(R \cup S)]$ is a sub-quasigroup of $Q$. If $R$ and $S$ do not intersect the results take the following form: I. $n \geqq r+s+\max (r, s)$. II. If $n=r+s+\max (r, s)$ then $r=s$ if and only if $Q \backslash(R \cup S)$ is a sub-quasigroup of $Q$. (Received July 6, 1956.)

640. J. H. Walter: Invariants for nonsemisimple algebras. Preliminary report.

Let $K$ be an algebraically closed field and $\mathfrak{A}$ be an algebra of finite rank over $K$ which possesses an identity. Let $\mathfrak{R}$ be the radical of $\mathfrak{A}$ and assume that $\mathfrak{A}-\mathfrak{R}$ is separable. Designate by $F_{1}, F_{2}, \cdots, F_{k}$ the irreducible representation modules of $\mathfrak{A}$. Let $U_{i}$ be a principal indecomposable representation module with $U_{i} / \mathfrak{N} U_{i}$ isomorphic to $F_{i}$. Designate by $V_{i j}$ the completely reducible representation space $\mathfrak{N}^{i} U_{i} / \mathfrak{N}^{i+1} U_{i}$. Let $H_{i, j k}$ be the vector space $\operatorname{Hom}_{\mathfrak{I}}\left(F_{i}, V_{j k}\right)$ of $\mathfrak{A}$-homomorphisms of $F_{i}$ into $V_{j k}$ and by $H_{i, j k}^{*}$ its dual. Then if $C^{1}\left(\mathfrak{R}, \operatorname{Hom}_{K}\left(F_{i}, F_{j}\right)\right)$ and $Z^{1}\left(\mathfrak{Y}, \operatorname{Hom}_{K}\left(F_{i}, F_{j}\right)\right.$ are the groups of 1 -chains and 1-cocycles defined by Hochschild, bilinear functions from $H_{i, j k}^{*} \times H_{m, j n}$ to $Z^{1}\left(\mathfrak{A}, \operatorname{Hom}_{K}\left(F_{m}, F_{i}\right)\right)$ and to certain factor groups of $C^{1}\left(\mathfrak{A}, \operatorname{Hom}_{K}\left(F_{m}, F_{i}\right)\right)$ are used to give a set of invariants which distinguish up to isomorphism $\mathfrak{A}$ from any other algebra $\mathfrak{R}$ with radical $\mathfrak{M}$ such that $\mathfrak{A}-\mathfrak{N}$ is isomorphic to $\mathfrak{R}-\mathfrak{M}$. (Received July 9 , 1956.)

\section{1t. Y. K. Wong: On matrices with nonnegative elements.}

Consider a square matrix $A=\left(a_{i j}\right)$ with elements in an ordered field. Assume that $a_{i j} \geqq 0$ for $i, j=1,2, \cdots, n$. If $u^{\prime} A \leqq u^{\prime}$ for some $u^{\prime}$ with positive components, then the adjoint $B=\left(b_{i j}\right)$ of $I-A$ is nonnegative, and $b_{i j} \leqq u_{j} u_{i}^{-b} b_{i i}$ where $i \neq j$, and $i, j=1, \cdots, n$. Let $A_{k}$ be the principal submatrix consisting of the elements in the first $k$ rows and columns of $A$. Then $\operatorname{det}\left(I-A_{k}\right)$ is nonnegative and is nonincreasing relative to $k$. By $\operatorname{det}\left(I-A_{n}\right) \geqq\left(1-a_{11}\right) \prod_{k}\left(1-a_{k k}-\sum_{i<k} u_{i} u_{k}^{-1} a_{i k}\right)$, where $k=2, \cdots, n$, gives a lower bound. Thus a sufficient condition for $\operatorname{det}\left(I-A_{n}\right)>0$ is that $u^{\prime} A<u^{\prime}$ for some $u>0$. By Gauss' method of elimination, we can reduce $I-A$ into the form $I-C$, where $C=\left(c_{i j}\right)$ is a triangle matrix with zeros below the principal diagonal. Then all the proper values of $A$ are less than unity in absolute value if and only if $1-c_{i i}>0$ for $i=1,2, \cdots, n$. (Received July 5,1956 .)

\section{ANALysis}

\section{2t. D. R. Anderson: On a generalization of the notion of a measura-} ble group.

In A. Weil's definition of measurable group (groupe mesuré) (P. Halmos, Measure theory, pp. 257-277) the requirement of left-translation invariance of the measure is equivalent to the weaker requirement that translation preserves measurability and sets of measure zero. (It is called a generalized measurable group if the weaker property is assumed.) It is shown that if the latter holds then an equivalent left-translation invariant measure exists. A new proof of the uniqueness of the left-invariant measure associated with a measurable group is also given. From these results follows a generalization of a theorem of MacKey and Loomis on representations of locally compact groups (Duke Journal of Mathematics vol. 19 (1952) pp. 641-645) where the topological assumptions are replaced by measure-theoretic assumptions. Also, one can show that a topological group is locally compact if and only if (1) the group uniform structure is complete, (2) the group is a generalized measurable group, and (3) all 
members of some fundamental system of neighborhoods of the identity are measurable. (Received August 17, 1956.)

\section{Richard Arens: Removing ideals by Banach algebra extension.}

An element $z$ of a commutative Banach algebra $A$ is called subregular if there is any extension $B$ of $A$ which has an inverse to $z$; this property implies that $z$ is not a zero divisor, even in the generalized sense. One establishes here the converse, by constructing an extension $B(t)$ in the following way. Having performed a scaler modification of $z$ (if needed) to insure that $\|a z\| \geqq\left\|a_{2}\right\|$ for all $a$ in $A$, one forms the set $A(w ; t)$ of all $f=a_{0}+a_{1} w+a_{2} w^{2}+\cdots$ where $\|f\|_{t}=\left\|a_{0}\right\|+\left\|a_{2}\right\| t^{2}+\cdots$ converges; and let $B(t)=A(w ; t) / J$ where $J$ is the closed ideal generated by $1-z w$. This $B(t)$ contains $A$ as soon as $t \geqq 1$, and thus solves the problem. This result can be reformulated in terms of removing points from spectra. When $A$ is semisimple and $z$ generates $A$, then (one can show that) $B(t)$ is semisimple, provided $t>\|\hat{z}\|_{\infty}$. (Received June 10, 1956.)

644. D. G. Aronson: The first boundary value problem for a linear parabolic equation containing a small parameter.

Let $\bar{R}$ be a closed region bounded by horizontal line segments $S_{1}, S_{4}$ and nowhere horizontal curves $S_{2}, S_{3}$. Consider in $\bar{R}$ the boundary value problem $\left(^{*}\right) L_{\epsilon}(u) \equiv \epsilon u_{x x}$ $+a(x, y) u_{x}-b(x, y) u_{y}+c(x, y) u=d(x, y), u$ specified on $S_{1}+\bar{S}_{2}+\bar{S}_{3}, \epsilon>0$, where the data is in $C^{\mathrm{IV}}$ and $b>0$. Let $\overline{\mathfrak{M}} \subset \bar{R}$ be bounded by $I<S_{i}(i=1,2,3), J \subset S_{2}+S_{3}$ $+\bar{S}_{4}-I$, and the characteristics of $\left({ }^{* *}\right) L_{0}(u) \equiv a u_{x}-b u_{y}+c u$ through their end points, where $I$ is a closed connected set such that no characteristic of $\left(^{* *}\right)$ starting there is tangent to $\bar{R}-R$ and $J$ is the image of $I$ under the characteristic mapping. Theorem: In any $\bar{M}$ the solution of $\left(^{*}\right)$ can be written $u(x, y, \epsilon)=v(x, y)+z_{2}(x, y, \epsilon)$ $+z_{3}(x, y, \epsilon)+w(x, y, \epsilon)$, where $v$ is the solution of $L_{0}(u)=d$ which assumes the given values of $u$ on $I, w=0$ on $\bar{M} \cap\left\{S_{1}+\bar{S}_{2}+\bar{S}_{3}\right\}$, and $w=O(\epsilon)$ uniformly as $\epsilon \rightarrow 0+$. If $J \bigcap S_{j}=0(j=2,3)$, then $z_{j} \equiv 0$; otherwise $z_{j}$ is a boundary layer term corresponding to $J \cap S_{j}$ and has the properties of the analogous term found in the elliptic case by Levinson (Annals of Math. vol. 51, pp. 428-445). The proof is based on Levinson's construction of the boundary layer term, the maximum principle for $L_{\epsilon}$, and the fact that $L_{\epsilon}(w)=O(\epsilon), w=0$ on $\bar{M} \cap\left\{S_{1}+\bar{S}_{2}+\bar{S}_{3}\right\}$, and $w$ uniformly bounded in $\bar{M}$, implies $w=0(\epsilon)$ inside $M$. (Received June 15, 1956.)

645. M. G. Arsove: Some remarks on potentials of linear mass distributions.

The remarks deal with properties of continuity and differentiability and their dependence on both the set (assumed to have finite positive linear measure) and on the mass distribution (assumed to be absolutely continuous with respect to the measure). Generalizations of the classical theorems are given, along with a number of counterexamples (e.g. there exists a rectifiable simple curve which at each point admits a tangent and has linear density one, but on which a uniform distribution of the unit mass gives rise to a discontinuous potential). (Received July $5,1956$.

\section{J. H. Barrett: A Prufer transformation for matrix differential} equations.

Let capital letters denote $n \times n$ square matrices and consider the matrix differential equation (I): $\left(P Y^{\prime}\right)^{\prime}+Q Y=0$, where on $a \leqq x<\infty, P(x)$ is nonsingular and $P(x)$ 
and $Q(x)$ are real, symmetric and continuous. It is shown that for every nontrivial solution $Y(x)$ of $(\mathrm{I})$, for which $Y(0)=0$, there exists a nonsingular differentiable matrix $R(x)$ and a symmetric continuous matrix $Q(x)$ such that $Y(x)=S^{*}[a, x ; Q] R(x)$ and $P(x) Y^{\prime}(x)=C^{*}[a, x ; Q] R(x)$, where $Z(x)=S[a, x ; Q]$ and $W(x)=C[a, x ; Q]$ are solutions of the system $Z^{\prime}=Q W, W^{\prime}=-Q Z, Z(a)=0, W(a)=E$ (identity). It is noted that for $n=1, S$ and $C$ are $\sin \int_{a}^{x} Q$ and $\cos \int_{a}^{x} Q$, respectively. For $n \geqq 1$ various "trigonometric" identities and inequalities are established for the matrices $S$ and $C$. (Received July $5,1956$. )

\section{7t. H. S. Bear and Bertram Yood: Multiplicative functionals on semi-groups of continuous functions.}

Let $S(X)$ be the multiplicative semi-group of all real continuous functions on a compact Hausdorff space $X$. Let $M(X)$ be the semi-group of all real continuous multilicative functions defined on $S(X)$ to the real number system. Let $X_{1}, X_{2}$ be compact Hausdorff spaces satisfying the first axiom of countability. It is shown that $X_{1}$ is homeomorphic to $X_{2}$ if and only if there exist a semi-group isomorphism of $M\left(X_{1}\right)$ onto $M\left(X_{2}\right)$. The case where the $X_{i}$ do not satisfy the countability axiom is also studied. (Received April 23, 1956.)

648t. Stefan Bergman: On singularities of solutions of certain differential equations in three variables. I.

In order to study real solutions of differential equations (1) $\widehat{\psi}_{x}+\widehat{\psi}_{y y}+\widehat{\psi}_{z z}+\widehat{F} \hat{\psi}$ $\equiv \psi_{X}-\psi_{z z^{*}}+F\left(Z, Z^{*}\right) \psi=0, \widehat{F}(y, z) \equiv F\left(Z, Z^{*}\right)$, the author introduces a special class $\mathcal{K}$ of complex solutions of (1) which possesses the property that $\psi\left(X, 0, Z^{*}\right)$ is a function of $X$ alone. (Here $X=x, Z=(z+i y) / 2, Z^{*}=-(z-i y) / 2 ; x, y, z$ are the Euclidean coordinates.) He defines an integral operator $\mathrm{P}[g(x, z)]$ transforming functions $g(X, Z)$ into solutions of (1). If in the domain $D$ of the $\left(X, Z, Z^{*}\right)$ space $\left|F\left(Z, Z^{*}\right)\right|$ $\leqq C<\infty,\left|g^{(k)}\right| \equiv\left|\partial^{k} g(X, Z) / \partial X^{k}\right| \leqq A k ! \epsilon_{k}$ then a solution of (1) possessing the property that (2) $\psi(X, Z, 0)=g(X, Z), \psi\left(X, 0, Z^{*}\right)=g(X, 0)$, exists provided that $\sum_{n=0}^{\infty}\left|Z Z^{*}\right|{ }^{n / 2} C^{-n / 2} \epsilon_{n} B_{n}\left[2 C^{1 / 2}\left|Z Z^{*}\right|{ }^{1 / 2}\right]<\infty$. ( $B_{n}$ are modified Bessel functions.) Let $J_{2}(k)\left(X, Z, Z^{*}\right)=\int_{0}^{z} \int_{0}^{z^{*}} F\left(Z_{2}, Z_{2}^{*}\right) \int_{0}^{z_{2}} \int_{0}^{z_{2}^{*}} F\left(Z_{1}, Z_{1}^{*}\right) g^{(k)}\left(X, Z_{1}\right) d Z_{1} d Z_{1}^{*} d Z d Z_{2}^{*}$ and $J_{n}^{(k)}$ be analogously formed sums of $2 n$-ply integrals. If $\psi=\sum_{\nu_{-0}}^{\infty} \sum_{k=0}^{\nu} J_{\nu}^{(k)}$ converges uniformly in a domain $D$, it represents a solution of (1) satisfying the conditions (2). The author derives the representation $\psi=\sum_{\nu=0}^{\infty} \sum_{k=0}^{\infty} \int_{0}^{Z} H^{(\nu, k)}\left(Z, Z^{*}, Z_{1}\right) g^{(k)}\left(X, Z_{1}\right) d Z_{1}$ for solutions of $(1) . \mathrm{H}^{(\nu, k)}$ depends only on $F$. (Received June 4,1956 .)

649t. Stefan Bergman. On singularities of solutions of certain differential equations in three variables. II.

This paper continues the author's investigations about the connection between the location and character of the singularities of a solution $\psi$ of a partial differential equation and the properties of a subsequence of coefficients of the series development of $\psi$. Let $L=\left[X-\sum_{m=0}^{M} a_{m} Z^{m}=0\right]$ where $a_{m}$ are constants. Choosing $g(X, Z)=(X$ $\left.-\sum_{m=0}^{M} a_{m} Z^{m}\right)^{-1}$ the author obtains solutions $\psi\left(X, Z, Z^{*}\right)=\mathrm{P}\left[\left(X=\sum_{m=0}^{M} a_{m} Z^{m}\right)^{-1}\right]$ $=\mathrm{P}\left[\sum_{\nu=0}^{M-1} \alpha_{\nu}(X)\left(Z-Z_{\nu}(X)\right)^{-1}\right]$ of $(1)$ of the class $\mathcal{K}$. They are singular along $L$. Necessary and sufficient conditions for the subsequence $\left\{A_{n m 0}\right\}, n, m=0,1,2, \ldots$ of the coefficients of a function element $\tau\left(X, Z, Z^{*}\right)=\sum_{n=0}^{\infty} \sum_{m=0}^{\infty} \sum_{r=0}^{\infty} A_{n m r} X^{n} Z^{m} Z^{* r}$ are determined in order that for $X_{0} \leqq X \leqq X_{1}, \tau=\psi+\phi$, where $\phi$ is an entire solution of (1). (The results obtained for solutions of the class $\mathcal{K}$ can be easily interpreted as analogous results for real solutions.) The author determines further the singu- 
larity manifolds $Z=Z_{\nu}(X), \nu=0,1, \cdots, M-1$ of $\tau$ in terms of the $\left\{A_{n, m, 0}\right\}$. These results can be generalized in many instances to the case of solutions $\psi$ $P\left[\left(\sum_{\nu=0}^{m} b_{\nu}(X) Z^{\nu}\right)^{-1}\right]$ e.g., if $b_{\nu}(X)$ are linear functions of $X$. (Received June 4,1956 .)

\section{G. U. Brauer: Sets of divergence of ordinary Dirichlet series.}

If $E$ is a set of type $F_{\sigma}$ on the $\tau$-axis $M$, and of logarithmic measure zero, then there exists an ordinary Dirichlet series $\sum_{n} a_{n} n^{-s}$ which diverges on $E$ and converges on $M-E$ (here $s=\sigma+i \tau$ ). The series has bounded partial sums on any bounded subset of $M$, and the function represented by this series is continuous on $M$. (Received July 2, 1956.)

651. T. S. Chihara: On a class of polynomials related to the Hermite polynomials.

The polynomials $R_{n}^{\nu}(x)=(-1)^{n} \exp \left(x^{2}\right)(d / d x)^{n} x^{\nu} \exp \left(-x^{2}\right)(n, \nu=0,1,2, \cdots)$ are considered. For $\nu=0,1$, these reduce to Hermite polynomials, while for $\nu \geqq 2$, $R_{n}^{\nu}(x)$ can be expressed as a simple linear combination of Hermite polynomials. This latter relation leads to the following "quasi-orthogonality" relation: $\int_{-\infty}^{\infty} \exp \left(-x^{2}\right)$ - $R_{n}^{\nu}(x) R_{m}^{\nu}(x) d x=0$ for $m \neq n \pm 2 j(j=0,1, \cdots,[\nu / 2])$. A simple generalization of this property to polynomials $q_{n}(x)$ satisfying a relation of the form $\int_{a}^{b} q_{n}(x) q_{m}(x) d \alpha(x)=0$ for $m \neq n \pm r j(j=0,1, \cdots, k)$ is briefly considered. Generating functions, recurrence formulas, differential equations, various representations, and simple bounds for the $R_{n}^{\nu}(x)$ are also obtained. (Received June $\left.28,1956.\right)$

652. P. C. Curtis, Jr.: Bounded directed families of self-adjoint elements in $A W^{*}$ algebras.

A C* algebra $A$ will be called a $\mathrm{BW}^{*}$ algebra if every bounded directed family of self adjoint elements in $A$ has a least upper bound in $A$. It is shown that $\mathrm{BW}^{*}$ algebras are a sub-class of $\mathrm{AW}^{*}$ algebras, and as a partial converse, an $\mathrm{AW}^{*}$ algebra of type I is a $\mathrm{BW}^{*}$ algebra. Let $A$ and $B$ be $\mathrm{BW}^{*}$ algebras with $A \subset B$. A will be called a $\mathrm{BW}^{*}$ subalgebra of $B$ if $A$ is a closed * subalgebra of $B$ and if the least upper bound (in $B$ ) of every bounded directed family of self adjoint elements of $A$ belongs to $A$. Let $A$ be a commutative subalgebra of $B$ and let $C$ be a maximal commutative subalgebra of $B$ containing $A$. By a suitable comparison between the structure spaces of $A$ and $C$, it can be shown that a necessary and sufficient condition for $A$ to be a BW* subalgebra of $B$ is for it to be an $\mathrm{AW}^{*}$ subalgebra of $B$. (Received July $5,1956$. )

\section{3t. M. M. Day: Means and ergodicity. II.}

The result of Abstract 606 can be used to strengthen and improve Theorem 4 of M and E, I (Trans. Amer. Math. Soc. vol. 69 (1950) pp. 276-281) to Theorem: If $\Sigma$ is an amenable semigroup and if $F$ is a bounded representation of $\Sigma$ over a Banach space $B$, then $F(\Sigma)$ is uniformly ergodic. (Received June 29, 1956.)

\section{4t. S. P. Diliberto: Periodic surfaces I.}

The notion of a periodic $k$-surface (a $k$-dimensional torus composed of trajectories is introduced in the study of systems of first order ordinary differential equations $\dot{x}=f(x, t, \cdots, t, \lambda)$ where $x=\left(x_{1}, \cdots, x_{n}\right), t, \lambda$ are real and $f\left(x, \theta_{1}, \cdots, \theta_{e}, \lambda\right)$ has period $w_{i}$ in $\theta_{i}$. This notion includes that of "periodic solution" and the KryloffBogilinboff "invariant curve"; and is shown to be fundamental in describing the physical phenomena of resonance, entrainment of frequency, and Plaat's phenomena. 
Several perturbation problems are solved. For the $K-B$ invariant curve problem for $\ddot{x}+w_{1}^{2} x=\lambda f(x, \dot{x}, t)$ where $f$ has period $2 \pi / w_{2}$ in $t ; a(r, \theta, t)=\cos w_{1} \theta f\left(r \sin w_{1} \theta, w_{1} r \cos \right.$ $\left.w_{1} \theta, t\right) ; b(r, \theta, t)=\partial a(r, \theta, t) / \partial r ; c(r, \theta, t)=\tan w_{1} \theta a(r, \theta, t) / r ; d(r, \theta, t)=c(r, \theta, t)$ - $\left(w_{1} w_{2} /(2 \pi)^{2}\right) \int_{0}^{2 \pi / w_{1}} \int 2 \pi / w_{2} c(r, \theta, t) d \theta d t$ we sharpen their theorem by reducing hypothesis to: smoothness of $f$; and for some $\rho_{0}(\neq 0) M_{6} a\left(\rho_{0}, t+\theta, t\right)=0, M_{t} d\left(\rho_{0}, t+\theta, t\right)=0$, and $M_{t} b\left(\rho_{0}, t+\theta, t\right) \neq 0$ (each for all $\left.\theta\right)$ where $M_{t}()=\lim _{T \rightarrow \infty}(1 / T) \int_{0}^{T}() d t$. (Received July 3, 1956.)

\section{5t. S. P. Diliberto: Periodic surfaces II.}

Let $\mathcal{C}$ be the Banach space of all continuous periodic (period $w_{1}$ ) real functions of the real variable $\theta_{1}$. If $\alpha_{i} \in \mathcal{C}(i=1,2)$ define $\left(\alpha_{1}+\alpha_{2}\right)\left(\theta_{1}\right)=\alpha_{1}\left(\theta_{1}\right)+\alpha_{2}\left(\theta_{1}\right) ;\|\alpha\|$ $=\max _{\theta}|\alpha(\theta)|$; and if $L$ a linear operator (on $\mathbb{C}$ into $\mathbb{C}$ ) let $\|L\|=\sup \|L \alpha\|$ for $\|\alpha\|=1$. Let $b\left(\theta_{1}, \theta_{2}\right)$ be continuous $\left(\theta_{1}, \theta_{2}\right)$ and periodic $w_{i}$ in $\theta_{i}(i=1,2) ; B\left(\theta_{1}, t\right)=\int_{0}^{l} b\left(s+\theta_{1}\right.$, $s) d s$ and $B\left(\theta_{1}\right)=B\left(\theta_{1}, w_{2}\right)$. Theorem: If $L$ is the linear operator on $C$ defined for $\alpha \in \mathcal{C}$ by $(L \alpha)\left(\theta_{1}\right)=\alpha\left(\theta_{1}+w_{2}\right)-\alpha\left(\theta_{1}\right) \operatorname{Exp}\left[B\left(\theta_{1}\right)\right]$, then $L^{-1}$ exists and is bounded if and only if $\left|\lim _{T \rightarrow \infty}(1 / T) B\left(\theta_{1}, T\right)\right|>0$ for all $\theta_{1}$. This result shows that the above strengthening of the $K-B$ theorem is sharp. (Received July 3, 1956.)

656t. Jim Douglas, Jr.: $A$ uniqueness theorem for the solution of a Stefan problem.

A Stefan problem is a free boundary problem associated with a parabolic differential equation. Numerous existence theorems have been given for such problems, but no complete uniqueness theorem has been proved. The object of this paper is to demonstrate the uniqueness of the solution of the following problem: $u_{x x}=f(u) u_{t}$, $0<x<x(t) ; u_{x}(0, t)=-a, t>0 ; u(x(t), t)=0, t>0 ; x(0)=0 ; d x(t) / d t=b-c u_{x}(x(t), t)$, $t>0$; where $x(t)$ is the position of the moving boundary at time $t, f(u)>0, f^{\prime}(u) \geqq 0$, and $a, b, c$ are positive constants. The paper will appear in the Proceedings. (Received July $5,1956$.

657. A. C. Downing, Jr.: Convergence of finite difference solutions of the multi-region diffusion problem.

The classical method of Courant, Friedrich, and Lewy for demonstrating the convergence of finite difference solutions of the Dirichlet problem is extended in this paper to the multi-region diffusion problem. Under appropriate uniqueness conditions, the prescribed discontinuities of the solution are shown to be satisfied almost everywhere in the limit as the mesh size of the finite difference solutions tend to zero. (Received July 9, 1956.)

\section{B. J. Eisenstadt and G. G. Lorentz (p): Boolean rings and Banach lattices.}

Let $\Phi(e) \geqq 0$ be a function defined on a Boolean $\sigma$-ring $B$. Necessary and sufficient conditions are given under which $B$ may be imbedded into a Banach lattice $X$ so that $\Phi(e)$ coincides with the norm of $X$ on $B$. Among such $X$ there is one with the largest norm; its elements are certain functions $x=x(t)$ from reals to $B . x, y$ are called covariant if all $x(t), y\left(t_{1}\right)$ are comparable. $B$ can be imbedded in an $X$ with the norm additive for positive covariant elements if and only if $\Phi$ is concave, i.e. satisfies $\Phi\left(e_{1} \cup e_{2}\right)$ $+\Phi\left(e_{1} \cap e_{2}\right) \leqq \Phi\left(e_{1}\right)+\Phi\left(e_{2}\right)$. In this case, the norm in the extremal extension $X=\Lambda \Phi$ of $B$ is given by $\|x\|=\int_{0}^{\infty} \Phi(|x(t)|) d t$, so that these $X$ are natural generalizations of $\Lambda$ spaces of one of the authors. These results allow a complete characterization of Banach 
lattices with a unit which are isomorphic to a general $\Lambda$-space. (Received July 5, 1956.)

659t. B. J. Eisenstadt and G. G. Lorentz: Functions of measures and $\Lambda$ spaces.

A Banach lattice may be realized as a collection of functions from the reals to a $\sigma$-Boolean algebra. [Freudenthal, Proc. Amsterdam Academy vol. 39 (1936) pp. 641651 ]. The norm is an extension of a positive (vanishing only at zero) increasing function $\Phi$ on the algebra. Necessary and sufficient conditions are given for $\Phi$ to be a real valued function of a positive measure if $\Phi$ is multiply subadditive [Lorentz, Canadian Journal of Math. vol. 4 (1952) pp. 455-462)] and if $\Phi$ is concave. Let $\mu$ be a fixed positive measure on the algebra. The notion of equimeasurability (with respect to $\mu$ ) is defined for positive measures. If $\Phi$ is concave and of the form $F(\mu)$, it is shown that $\Phi=\sup \eta_{r}$ where $\eta_{r}$ runs through a fixed class of equimeasurable positive measures. Furthermore, $\eta_{r}(e)=\int_{e} f_{r}(t) d \mu(t)$ where the $f_{r}$ are equimeasurable (in the usual sense) positive functions on a representation space $E$. If, in addition, the Banach lattice is a $\Lambda_{\Phi}$ space, then under this representation an element $x$ of the lattice becomes a real function $x(t)$ on $E$ with the norm $\sup f_{r} \int_{E}|x(t)| f_{r}(t) d \mu(t)$. (Received July 5,1956 .)

660t. Evelyn Frank: A new class of continued fraction expansions for the ratios of Heine functions.

A new class of continued fraction expansions for the ratios of two Heine functions is described in detail. A number of ratios of Heine functions are expanded, and the convergence of the corresponding continued fractions is determined as well as the functions to which these continued fractions converge in certain regions. Recurrence relations for the numerators and denominators of the approximants of these continued fractions are derived. Certain special continued fraction expansions are found, and many special relations are obtained between functions, finite continued fractions and infinite continued fractions. (Received July 6, 1956.)

\section{W. B. Fulks: Heat conduction in general regions.}

Let $R$ be a bounded region in the $x t$ plane, and $f$ a bounded function defined on the boundary $B$ of $R$. The method of $O$. Perron is applied to the problem of finding a solution to the Dirichlet problem for the heat equation: to find a solution of $u_{t}=u_{x x}$ in $R$ and $u=f$ on $B$. Sub- and super-parabolic functions are defined and used in a manner analogous to the use of sub- and super-harmonic functions. (See also Sternberg, Math. Ann. (1929) and Petrowsky, Comp. Math. (1934).) The concept of thermal capacity zero is defined and the following theorem is proved: if $u_{1}$ and $u_{2}$ be two solutions of $u_{t}=u_{x x}$ in $R$ and have the same boundary values on $B$ except possibly for a set $E$ of thermal capacity zero, then $u_{1} \equiv u_{2}$. (Received July $5,1956$. )

662t. I. S. Gál: On the fundamental theorems of the differential and integral calculus.

Let the real function $f$ be defined in the finite closed interval $[a, b]$ and let $\Lambda^{-}(x)$ and $\Lambda^{+}(x)$ denote the limit superior of $f$ at $x$ from the left and from the right respectively. Denote by $\mu(t)$ the exterior or the interior measure of the set where $D^{+} f<t$ according as $t<0$ or $t>0$. A property is said to hold nearly everywhere if it holds everywhere except possibly on a countable set of points. It is proved that: I. If $\Lambda^{-}(x) \leqq f(x) \leqq \Lambda^{+}(x)$ everywhere and $D^{+} f>-\infty$ nearly everywhere then $f(b)-f(a)$ 
$\geqq \int t d \mu$ with $-\infty<t \leqq 0$. Hence if $D^{+} f \geqq 0$ almost everywhere then $f$ is increasing in $[a, b]$. II. If $f$ is continuous in $[a, b]$ and $D^{+} f>-\infty$ nearly everywhere then $f(b)-f(a)$ $\geqq t d \mu$ with $-\infty<t<+\infty$ whenever $\int t d \mu$ with $-\infty<t \leqq 0$ converges. De la Vallee Poussin's decomposition theorem is a simple consequence of I and II. Only the elements of the theory of exterior measure and the compactness of $[a, b]$ are used in the proofs. (Received June 29, 1956.)

\section{3t. B. R. Gelbaum: Conditional and unconditional convergence in} Banach spaces.

The countable direct product $G_{\infty}$ of the group of order two with itself is used to investigate conditional and unconditional convergence in Banach spaces: (i) If $\left\{x_{n} ; X_{n}\right\}$ is a basis, then $\sum_{1}^{\infty} \epsilon_{n}(g) X_{n}(x) x_{n},\left(\epsilon_{n}(g)= \pm 1\right)$, converges for every $x$ for all $g$ in $G_{\infty}$ or for a set of $g$ of measure 0 ; (ii) Let $R(x)=\left\{y \mid y=\sum_{1}^{\infty} \epsilon_{n}(g) X_{n}(x) x_{n}, g\right.$ in $\left.G_{\infty}\right\}$. Then $\left\{x_{n} ; X_{n}\right\}$ is an unconditional basis if and only if $R(x)$ has a compact closure in either the weak or norm topologies; (iii) Let $H=\left\{x \mid \sum_{1}^{\infty} \epsilon_{n}(g) X_{n}(x) x_{n}\right.$ converges for all $g$ in $\left.G_{\infty}\right\}$. Then $H$ is the whole Banach space or $H$ is of the first category. (Received May 14, 1956.)

\section{4t. B. R. Gelbaum: Notes on Banach spaces and bases.}

A collection of theorems on the phenomena associated with bases in Banach spaces is offered. Among other results are the following, some of which are stated in Banach's book without proof or reference: (i) Every separable Banach space has a basis if and only if every Banach space which is the linear image of a Banach space with a basis has a basis. (ii) Every separable Banach space is the linear image of a Banach space with a basis. (iii) If $\left\{x_{n} ; X_{n}\right\}$ is a basis, there are constants $a_{n}$ such that the $\rho_{n}$ $=\sum_{1}^{n} a_{m} x_{m}$ form a basis. Examples and special cases are discussed. (Received May 14, 1956.)

\section{5t. Casper Goffman: Convergence in norm of integral means.}

Let $X$ be a Banach lattice of summable functions on the closed interval $[0,1]$, such that if $x=x(t) \in X$ then, for every $u, x_{u}=x(t+u) \in X$ and for every $\epsilon>0$ there is a $\delta>0$ such that $|u-v|<\delta$ implies $\left\|x_{u}-x_{v}\right\|<\epsilon$. It is shown that the integral means $x^{h}=h^{-1} \int_{0}^{h} x(t+u) d u$ belong to $X$ and that $\lim \left\|x-x^{h}\right\|=0$, for every $x \in X$. This furnishes a rigorous version, for this special kernel system, of a heuristic theorem stated by S. Bochner (Harmonic Analysis and the Theory of Probability, Berkeley, 1955, p. 9). The lattice property seems to overcome the absence of an analogue to the Hölder inequality, which is used in the proof for $L_{p}$. (Sponsored by National Science Foundation grant no. NSF G-2267). (Received June 6, 1956).

666t. Casper Goffman: Compatible semi-norms in a vector lattice. I.

A locally convex topology $\tau$ for a vector space $X$ is compatible with a lattice ordering $\omega$ for $X$ if $\tau$ may be given by semi norms $P_{\alpha}$ for which $|x| \geqq|y|$ implies $P_{\alpha}(x) \geqq P_{\alpha}(y)$. With the notations $X_{\tau}$ for the topological vector space, $X_{\tau}^{\prime}$ for its dual, and $X_{\omega}$ for the vector lattice, $X_{\omega}^{\prime}$ for its dual of bounded linear forms, the following is obtained: If $\tau$ is compatible with $\omega$ then $X_{\tau}^{\prime} \subset X_{\omega}^{\prime}$ and if $\tau$ is the finest topology compatible with $\omega$ then $X_{\tau}^{\prime}=X_{\omega}^{\prime}$. Hence, if $X_{\tau}$ is a Mackey space $(\tau$ compatible with $\omega)$ for which $X_{\tau}^{\prime}=X_{\omega}^{\prime}$ then $\tau$ is the finest topology compatible with $\omega$. For Banach spaces, the following is then obtained: A given vector lattice may be normed as a Banach lattice in at most one way. In particular, the classical norms used for various real 
function spaces thus appear as the only "reasonable" choices. (Sponsored by National Science Foundation Grant No. NSF G-2267). (Received May 14, 1956.)

667. A. A. Goldstein: On the Tchebycheff and least (2q) th approximation of an overdetermined system of linear equations.

Let $A=\left\|a_{i j}\right\|$ be an $m \times n$ matrix $m>n$ which has no vanishing $n \times n$ determinants and let $X=\left\|x_{j}\right\|$ be a vector whose domain is a closed bounded subset $E$ of the $n$ dimensional Euclidean vector space $E_{n}$. Let $B=\left\|b_{i}\right\|$ and $Y=\left\|y_{i}\right\|$ be vectors in $E_{m}$. If the transform $A X=Y$ of $X$ lies closest to $B$ in the metric $1 . \sup _{i}\left|y_{i}-b_{i}\right|$ or 2 . $\left[\sum_{i}\left(y_{i}-b_{i}\right)^{2 q}\right]^{1 / 2 q}$ then the solution vector will be called Tchebycheff or least $(2 q)$ th and denoted by $\bar{X}$ or $X^{\prime}$ respectively. We have that the $X \in E$ which satisfy $\sup _{i}\left|\sum_{j} a_{i j} x_{j}-b_{i}\right| \leqq K$ and $\sum_{i}\left(\sum_{j} a_{i j} x_{j}-b_{i}\right)^{2 q} \leqq K^{2 q}$ are convex bodies, and that for $q$ sufficiently large the $\bar{X}$ and $X^{\prime}$ lie arbitrarily close. This suggests the method of descent to find $\bar{X}$ or $X^{\prime}$. Let $g(x)=k, m<k \leqq K$ describe a convex body, where $m$ is the minimum of $g(x)$. Define a sequence $g\left(x_{p}\right)$ as follows: given $Z_{p} \in E$ an arbitrary vector let $x_{p+1}$ be the point on which $g(x)$ assumes a minimum along the ray through $x_{p}$ parallel to $Z_{p}$. Let $E^{\prime}$ be the set of all rays through $x_{p}$ which cut the assemblage of points $g(x)<g\left(x_{p}\right)$. If $Z_{p}$ is any sequence such that the rays through $x_{p}$ parallel to $Z_{p}$ are frequently in $E^{\prime}$ then $g\left(x_{p}\right)$ converges to the minimum of $g(x)$ and there exists a unique $x$ for the minimum of $g(x)$. (Received July 9, 1956.)

668t. J. K. Hale: On a class of linear differential equations with periodic coefficients.

Consider the system of linear differential equations (1) $y^{\prime \prime}+A(\lambda) y=\lambda \phi(t, \lambda) y$ $+\lambda \psi(t, \lambda) y^{\prime}$, where $\lambda$ is a real parameter, $y=\left(y_{1}, \cdots, y_{n}\right), A(\lambda)=\operatorname{diag}\left(\sigma_{1}^{2}, \cdots, \sigma_{n}^{2}\right)$, $\phi$ and $\psi$ are $n \times n$ matrices whose elements are real, periodic functions of $t$ of period $T=2 \pi / \omega$, are $L$-integrable in $[0, T]$, are analytic in $\lambda$ and have mean value zero. Further, suppose that each $\sigma_{j}^{2}(\lambda), j=1,2, \cdots, n$, is a real positive analytic function of $\lambda$ with $\sigma_{j}(0) \not \equiv \sigma_{h}(0),(\bmod \omega i), j \neq h, j, h=1,2, \cdots, n$. By employing a method of successive approximations introduced by L. Cesari [Atti Accad. Italia, (6) vol. 11 (1940) pp. 633-692], the following theorem is proved: If $\phi=\left\|\phi_{i j}\right\|, \psi=\left\|\psi_{i j}\right\|$ where $\phi_{i j}, \psi_{i j}, i, j=1,2$, are matrices with $\phi_{11}$ and $\psi_{11}$ of dimension $\mu \times \mu, 0 \leqq \mu \leqq n$, and if $\phi_{11}, \phi_{22}, \psi_{12}, \psi_{21}$ are even in $t, \phi_{12}, \phi_{21}, \psi_{11}, \psi_{22}$, are odd in $t$, then, for $|\lambda|$ sufficiently small, all the absolutely continuous solutions of (1) are bounded in $(-\infty,+\infty)$. (Received July $2,1956$.

669. J. K. Hale: A new sufficient condition for periodic solutions of a class of weakly nonlinear differential equations.

A vector function $f(x, t)$ belongs to $A[\omega]$ if, in a neighborhood $U$ of the origin ( $U$ independent of $t$ ), each component of $f$ has a power series expansion convergent in $U$ whose coefficients are $L$-integrable functions of $t$ of period $2 \pi / \omega$. Consider a system of equations (1) $y^{\prime \prime}+D y=\epsilon f\left(y, y^{\prime}, \epsilon, t\right)$, where $y=\left(y_{1}, \cdots, y_{n}\right), f=\left(f_{1}, \cdots, f_{n}\right)$, $D=\operatorname{diag}\left(d_{1}^{2}, \cdots, d_{n}^{2}\right), \epsilon$ real $, d_{1}, \cdots, d_{n}$ positive, and $f \in A[\omega]$. Let $m$ be any integer, $0 \leqq m \leqq n$, and $y=(u, w), u=\left(y_{1}, \cdots, y_{m}\right), w=\left(y_{m+1}, \cdots, y_{n}\right), a=\left(a_{1}, \cdots, a_{n}\right)$, $b=\left(b_{1}, \cdots, b_{n}\right), c=\left(c_{1}, \cdots, c_{n}\right), d=\left(d_{1}, \cdots, d_{n}\right)$, where the numbers $a_{j}, b_{j}$ are positive integers. By employing a method which has been successively developed by $\mathrm{L}$. Cesari, R. A. Gambill, and J. K. Hale, the following theorem is proved: If $f_{j}(u,-w$, $\left.-u^{\prime}, w^{\prime}, \epsilon ;-t\right)=\lambda_{j} f_{j}\left(u, w, u^{\prime}, w^{\prime}, \epsilon ; t\right), \lambda_{j}=+1$ if $j=1, \cdots, m, \lambda_{j}=-1$ if $j=m+1$, $\cdots, n$, then real functions $B_{h}(a, b, c, d, \omega, \epsilon), h=1, \cdots, n$, analytic in $\epsilon$ for $|\epsilon|$ 
small, are determined such that, for the system of equations (2) $a_{h} \omega / b_{h}+\epsilon B_{h}=d_{h}$, $h=1, \cdots, n$, has solutions for real non-zero $d, c$, and $|\epsilon|$ small, then system (1) has a solution $y(\epsilon, t) \in A\left[\omega / b_{1} \cdots b_{n}\right]$ of the form $y_{j}(0, t)=c_{j} \cos \left(a_{j} \omega t / b_{j}\right), j=1,2, \cdots, m$, $y_{j}(0, t)=c_{j} \sin \left(a_{j} \omega t / b_{j}\right), j=m+1, \cdots, n$, and $y_{j}(\epsilon,-t)=\lambda_{j} y_{j}(\epsilon, t), j=1, \cdots, n$. Solutions for system (2) can be assured even in cases where classical known conditions fail. (Received July 2, 1956.)

\section{John Harton, Jr.: Extremal problems for real star mappings.}

Let $S=$ the family of functions $f(z)=z+a_{2} z^{2}+a_{3} z^{3}+\cdots$ which are regular and univalent for $|z|<1$, map the unit circle onto star-shaped regions and for which $a_{2}, a_{3}, \ldots$ are real. Let $B, C$ and $\alpha$ be complex numbers such that $|B|+|C|>0$ and $0<|\alpha|<1$. Consider the maximum possible value of the real part of $B \log f^{\prime}(\alpha)$ $+C \log [f(\alpha) / \alpha]$ for $f \in S$. A function of $S$ which maximizes the above expression is called an extremal function. It is shown that every extremal function is a member of $S$ which maps the unit circle onto the $\omega$-plane with, at most, four radial slits. That this theorem is sharp is shown by taking particular values of $B, C$ and $\alpha$ for which extremals with one up to four radial slits are found. For a real explicit formulas are obtained for the extremal functions and in this case each has at most two radial slits. The problem is attacked by means of an approximation theorem which reduces the problem to that of maximizing a certain sum. This method is similar to that used by Robinson (Proc. Amer. Math. Soc. vol. 6 (1955) pp. 364-377). (Received June 27, 1956.)

\section{L. J. Heider: The Myers' $F_{T}$ functionals as integrals.}

A Banach space $B$ is susceptible of an ordering under which it becomes an abstract ( $A L) L$ space, if and only if it contains a subset $T$ closed under addition, and such that for each element $a$ in $B$ there exist uniquely elements $a^{+}, a^{-}$in $T$ with $a=a^{+}-a^{-}$ and $\|a\|=\left\|a^{+}\right\|+\left\|a^{-}\right\|$, while $a=b-c$ with $b, c$ in $T$ implies $\left\|b-a^{+}\right\|=\|b\|-\|a\|$. The resulting $A L$ space has a weak unit if and only if $T$ contains an element $e$ such that $\|a-e\|<\|a\|+\|e\|$ for all $a$ in $T$. A subset $T$ of $B$ with the above properties is a Myers' $T$-set, and if one $T$-set of $B$ has the above properties, so does any other. Let $T_{s}, s \in S$, denote the collection of all $T$-sets in a potential $A L$ space $B$, and let $F_{s}$ denote the $F_{T}$ functional corresponding to $T_{s}$. In the representation of the $A L$ space $\left(B, T_{s_{0}}\right), T_{s_{0}}$ fixed, as the space of integrable functions over a measure space $(Y, S, \mu)$, for all $a$ in $\left(B, T_{s_{0}}\right)$ one has $F_{s_{0}}(a)=\int a d \mu$, while $\left(F_{s_{0}}[a]+F_{s}[a]\right) / 2=\int_{s} a d \mu$ under the 1-1 correspondence between measurable subsets $s \in S$ of $Y$ and $T$-sets $T_{s}, s \in S$, of $B$. In the representation of $\left(B, T_{s}\right), s \neq s_{0}$, the same space $(Y, S, \mu)$ is used, but the functions on $Y$ representing a given element of $B$ first as in $\left(B, T_{s_{0}}\right)$ and then as in $\left(B, T_{s}\right)$ differ by a change of sign at each point of the measurable subset $s \in S$ of $Y$ corresponding to the $T$-set $T_{\mathrm{s}}$. (Received July 2,1956.)

\section{2t. J. A. Hummel: A counterexample to the Poincaré inequality.}

If $D$ is a bounded plane domain with a sufficiently regular boundary, then there exists a constant $K$ such that for any continuous real function $\phi$ with $\iint_{D} \phi d x d y=0$, the Poincare inequality $\iint_{D} \phi^{2} d x d y \leqq K \iint_{D}\left(\phi_{x}^{2}+\phi_{y}^{2}\right) d x d y$ holds (cf. Courant and Hilbert, Methoden der Mathematischen Physik, vol. II). It is shown that this inequality does not hold for all bounded plane domains even if $\phi$ is restricted to be harmonic. In the $w$-plane $(w=u+i v)$, let $\Delta=\left\{w: 1<u<\infty, 1 /(u+2 \pi)^{2}<v<1 / u^{2}\right\}$. Then the counterexample is given by the domain $D$ which is the image of $\Delta$ under the mapping 
$z=e^{i w}$ and $\phi=u-c$, where $c$ is to be chosen to make $\iint_{D} \phi d x d y=0$. (Received July 5 , 1956.)

673. J. A. Hummel: Complete orthonormal sequences of functions uniformly small on a subset.

If $G$ is a region of the complex plane and if $f$ and $g$ are two complex valued functions, set $(f, g)_{G}=\iint_{G} f \bar{g} d x d y,\|f\|_{G}=(f, f)_{\epsilon}^{1 / 2}$. Let $D$ be a bounded plane domain and $L^{2}(D)$ the Hilbert space of all analytic functions with $\|f\|_{D}<\infty$. Let $G$ be a domain in $D$ with $\bar{G} \subset D$ and let $\left\{\psi_{n}\right\}$ be a doubly orthogonal sequence in $G$ and $D$ (cf., Bergman, The kernel function, Mathematical Surveys, no. 5, Amer. Math. Soc., 1950, pp. 14-17), i.e., $\left\{\psi_{n}\right\}$ is complete in $L^{2}(D),\left(\psi_{n}, \psi_{m}\right)_{D}=\delta_{n m},\left(\psi_{n}, \psi_{m}\right)_{G}=\lambda_{n} \delta_{n m}$, and $\sum \lambda_{n}=M^{2}<\infty$. Let $\left(\epsilon_{i j}\right.$ ) be an $N \times N$ matrix (where $N=2^{p}$ for some integer $p$ ) with each $\epsilon_{i j}= \pm 1$ and $\sum_{j} \epsilon_{i j} \epsilon_{k j}=0$ for $i \neq k$. Set $\phi_{i}=(1 / N)^{1 / 2} \sum_{i=1} \epsilon_{i j} \psi_{j}$ for $i \leqq N$ and $\phi_{i}=\psi_{i}$ for $i>N$. Then $\left\{\phi_{n}\right\}$ is a complete orthonormal set in $L^{2}(D)$, but given $\epsilon>0$, for $N$ sufficiently large, $\left\|\phi_{n}\right\|_{G<\epsilon}$ for all $n$. Using this result, it is easily proved that: If $K$ is a compact subset of $D$, then given $\epsilon>0$ there exists a complete orthonormal set $\left\{\phi_{n}\right\}$ in $L^{2}(D)$ such that $\left|\phi_{n}(z)\right|<\epsilon$ for all $n$ and all $z \in K$. This shows in particular that if the Bergman kernel function is to be approximated by a finite number of terms of its series expansion, the number of terms required cannot be given independently of the sequence used. (Received July $5,1956$.

674. Meyer Jerison: The set of all generalized limits of bounded sequences. II.

Let $L$ denote the set of all generalized (Banach) limits defined on the space $(m)$ of bounded sequences. $L$ is a weak ${ }^{*}$-compact convex subset of the conjugate space of $(m)$, and in an earlier note [Bull. Amer. Math. Soc. Abstract 59-6-625] a set $Q$ of elements of $L$ was constructed with the property that the closed convex hull of $Q$ is $L$. For any $x=\left(\xi_{1}, \xi_{2}, \cdots\right)$ in $(m)$, it is possible to find $q(x)=\sup _{f} \in L f(x)$ by calculating $\sup _{f} \in{ }_{a} f(x)$. It turns out to be $q(x)=\lim _{n \rightarrow \infty} \lim \sup _{k \rightarrow \infty} n^{-1} \sum_{i=0}^{n-1} \xi_{k+i}$, which is much simpler than the formula given by Banach (Théorie des opérations linéaires, pp. 3334). (Received July 6, 1956.)

675t. E. S. Johnson and C. T. Taam: On the solutions of nonlinear differential equations II.

Let $p(t)$ and $q(t)$ be real Lebesgue-measurable functions having positive lower and upper bounds on $0 \leqq t<\infty$. Denote by $x=x(t, E)$ the solution of $(\mathrm{NL}) x^{\prime \prime}+p x-2 q x^{3}$ $=0$ satisfying $\left(^{*}\right) x(0)=0, x^{\prime}(0)=E>0$. The starting point in this paper is this result: (I). If $0<E_{1}<E_{2}$, then $x\left(t, E_{1}\right)<x\left(t, E_{2}\right)$ for all positive $t$ as far as $x\left(t, E_{1}\right)$ remains non-negative. (I) is proved by an argument resembling the proof of Sturm's comparison theorem. (I) is used to prove (II): there exists a positive constant $E_{0}$ such that for $0<E<E_{0}, x(t, E)$ has a first positive zero $T(E)$ which increases with $E$ and tends to $\infty$ or to a positive limit according $E \rightarrow E_{0}-$ or $E \rightarrow 0+$. The solution $x\left(t, E_{0}\right)$ exists and is bounded for all $t \geqq 0$ and has no positive zero. For $E_{0}<E<\infty, x(t, E)$ has no positive zero and tends to $\infty$ as $t$ tends to some finite positive number. Under the additional assumption that $p$ and $q$ are even periodic functions with a common period then, as a consequence of (II), equation (NL) possesses various oscillatory periodic solutions. Solutions satisfying different initial conditions also are discussed. A comparison theorem of Sturmian type is also obtained. This work was supported by the Office of Ordnance Research. (Received July 9, 1956.) 
676. R. V. Kadison (p) and I. M. Singer: Three test problems in operator theory.

The following three problems were suggested to us in conversation by I. Kaplansky as test problems for the adequacy of a theory of unitary equivalence of operators on a Hilbert space (rephrased, these are the test problems of his Infinite Abelian groups): (1) With $A$ and $B$ operators on $H$ and $K$, does the unitary equivalence of $\operatorname{diag}(A, A)$ and $\operatorname{diag}(B, B)$ acting in the usual way on $H \oplus H$ and $K \oplus K$ imply the unitary equivalence of $A$ and $B$ ? (2) Does the unitary equivalence of diag $(A, B)$ and diag $(A, C)$ imply that of $B$ and $C$ ? (3) If $A$ and $B$ are unitarily equivalent to direct summands of each other are they unitarily equivalent? Using the theory of rings of operators, it is shown that the answer to Problems 1 and 3 is "Yes." Problem 2 has a negative answer, in general, but has an affirmative answer when the operators discussed generate rings of finite type with finite commutants. (Received July 5, 1956.)

677. N. D. Kazarinoff (p) and R. W. McKelvey: A note on the asymptotic behavior of Laguerre polynomials and functions for large $n$.

Tricomi [Ann. Mat. Pura Appl. (4) vol. 28 (1949) pp. 263-289] has derived a series of the form $n ! e^{-x / 2} L_{n}^{\alpha}(x)=\sum_{m=0}^{\infty} A_{n}(\nu) J_{\alpha+m}\left[(\nu x)^{1 / 2}\right](x / \nu)^{m / 2}, \nu=4 n+2 \alpha+2$, which is an asymptotic series in $n$ for $L_{n}^{\alpha}(x)$ ( $x$ complex and $\alpha$ real) when $|x|<C n^{1 / 3}$. Recent results of McKelvey yield a different expansion which asymptotically represents $L_{n}^{\alpha}(x)$ ( $x$ and $\alpha$ complex) at least when $|x|<4\left(2-3^{1 / 2}\right)^{2} n-\epsilon, \epsilon>0$ and small. It is of the form $c_{1}(n) J_{\alpha}(\xi) \sum_{j=0}^{\infty} a_{j}(x) n^{-i}+c_{2}(n) J_{\alpha}^{\prime}(\xi) \sum_{j=0}^{\infty} b_{j}(x) n^{-i}$, where $\xi(x, n), a_{j}(x)$, and $b_{j}(x)$ are elementary functions or integrals of elementary functions. The result may also be stated for Laguerre functions. (Received July 3, 1956.)

678t. C. E. Kerr: On the Tchebycheff approximation of a continuous function of $M$-variables by a linear combination of continuous functions of $M$-variables.

Define $\mathcal{C}$ to be the family of continuous functions with continuous first derivatives with at most a finite number of oscillations on the closed interval $[\alpha, \beta]$. Let $a_{i}(t) \in \mathcal{C}$ $i=1,2,3, \cdots, n$ and $b(t) \in \mathcal{C}$ be $n+1$ linearly independent functions and let $x=\left(x_{1}, x_{2}, \cdots x_{n}\right)$ be a vector from the $n$-dimensional Euclidean vector space, $E_{n}$. Define the Tchebycheff solution vector $\bar{x}$ as the $\bar{x} \in E_{n}$ such that $\sup _{t}\left|\sum_{i} a_{i}(t) \bar{x}_{i}-b(t)\right|$ is minimized. It is shown that the problem of finding $\bar{x}$ is equivalent to minimizing a convex function $M(x)$ defined on a bounded closed convex set $E \rightarrow E_{n}$; a constructive proof can be made using the method of descent to show that this function has an absolute minimum given at a unique vector $\bar{x} \in E_{n}$. It is shown that when an analogous class of functions $B$ is defined for a continuous variable of a higher dimension and the same problem is proposed the method of descent again implies the existence and uniqueness of a solution vector $\bar{x}$. (Received July 9, 1956.)

679t. Erwin Kreyszig: Coefficient problems of solutions of partial differential equations of the second order I.

Solutions (1) $u\left(z, z^{*}\right)=\sum_{m, n=0}^{\infty} u_{m n} z^{m} z^{* n}$ of (2) $u_{z z^{*}}+a\left(z, z^{*}\right) u_{z}+b\left(z, z^{*}\right) u_{z^{*}}+c\left(z, z^{*}\right) u$ $=0(a, b, c$ entire) can be represented by Bergman operators of the first kind: (3) $u\left(z, z^{*}\right)=p(g) \equiv \exp \left(-\int_{0}^{z *} a(z, t) d t\right) g(z)+\sum_{n=1}^{\infty} q_{n}\left(z, z^{*}\right) \int_{0}^{z} \int_{0}^{z}{ }_{1} \cdots \int_{0}^{z}{ }_{n-1} g\left(z_{n}\right) d z_{n} \cdots d z_{1}$ where $q_{n}(z, 0)=0, n=1,2, \cdots$ and $g(z)$ is an analytic function regular at the origin, cf. S. Bergman, Trans. Amer. Math. Soc. vol. 57 (1945) p. 299 ff. By means of (3) 
theorems on analytic functions can be formulated as theorems on solutions of (2). In this way various properties of $u\left(z, z^{*}\right)$ can be obtained if the sequence $\left(u_{m 0}\right)$ is known, cf. (1). In consequence of the properties of the above Bergman operators other coefficients $u_{m n}$ do not occur in theorems of this type. Obviously, information on the behavior of $u\left(z, z^{*}\right)$ should be obtained also from other sequences $\left(u_{m n}\right), n>0$ and fixed. Indeed by inserting (4) $u\left(, z, z^{*}\right)=\sum_{n=0}^{\infty} u_{n}(z) z^{*^{n}}, u_{n}(z)=\sum_{m=0}^{\infty} u_{m n} z^{m}$, into (2) a system $S_{n}$ of ordinary differential equation is obtained which yields relations between the sequences $\left(u_{m n}\right), n>0$ and fixed, and $\left(u_{m 0}\right)$. Cf. the following abstract. (Received July 23, 1956.)

\section{0t. Erwin Kreyszig: Coefficient problems of solutions of partial differential equations of the second order II.}

The ordinary differential equations of the system $S_{n}$ (cf. the preceding abstract) are linear and of the first order. They involve the functions $u_{0}(z), u_{1}(z), \cdots, u_{n}(z)$ and thus yield relations between $\left(u_{m 0}\right)$ and $\left(u_{m n}\right), n>0$ and fixed. There are two possibilities: (A) The system $S_{n}$ may be considered in its original form. (B) It may be transformed into a single ordinary linear differential equation which involves only the functions $u_{0}(z)$ and $u_{n}(z)$ and whose order does not exceed $n$. Since (B) leads to relatively complicated conditions, (A) is preferable in many cases of partial differential equations (1) (cf. the preceding abstract) which are of practical importance. At a singular point $z_{0}$ of $u_{n}(z)$ the function $u_{0}(z)$ is singular while $u_{\alpha}(z), 0<\alpha<n$, may or may not be singular at $z_{0}$. Conversely, a singular point of $u_{0}(z)$ may correspond to a regular point of $u_{n}(z)$. However conditions can be obtained in order that a singular point of $u_{0}(z)$ be a singular point of $u_{n}(z)$. In this way the domain of regularity and other properties of solutions $u\left(z, z^{*}\right)$ of (1) (cf. the preceding abstract) can be determined if a sequence $\left(u_{m n}\right), n>0$ and fixed, of the coefficients of the corresponding power series development is known. (Received July 23, 1956.)

681t. Erwin Kreyszig: On solutions of partial differential equations generated by Bergman operators.

In a recent paper (Journal of Rational Mechanics and Analysis vol. 4 (1955) pp. 907-923) necessary and sufficient conditions were obtained in order that solutions $u\left(z, z^{*}\right)$ of $(1) u_{z z^{*}}+B\left(z, z^{*}\right) u_{z^{*}}+C\left(z, z^{*}\right) u=0$ can be generated by a Bergman operator (cf. Bergman, Trans. Amer. Math. Soc. vol. 53 (1943) pp. 130-155) in the form (2) $u\left(z, z^{*}\right)=\int_{-1}^{1} E\left(z, z^{*}, t\right) f\left(z\left(1-t^{2}\right) / 2\right)\left(1-t^{2}\right)^{-1 / 2} d t$ where the "generating function" $E$ is of the form (3) $E\left(z, z^{*}, t\right)=\exp \left(Q\left(z, z^{*}, t\right)\right), Q=\sum_{\alpha=1}^{m} q_{\alpha}\left(z, z^{*}\right) t^{\alpha}$ and the "associated function" $f(z)$ is an analytic function regular at the origin. Theorem: Solutions (2) of (1) corresponding to generating functions (3) and meromorphic associated functions $f(z)=\left(z-z_{0}\right)^{-n}, z_{0} \neq 0, n=1,2, \cdots$ satisfy also a linear ordinary differential equation in $z_{1}=\left(z-z^{*}\right) / 2$ with algebraic coefficients. The order $k$ of this equation is independent of $n$; there always exists such an equation for which $k \leqq m+3$. In particular, if $Q$ is an even function of $t$ then $k \leqq m / 2+2$. This result and the method of the proof is different from that in the case of regular associated functions $f(z)=z^{n}$, $n=0,1, \cdots$ which was investigated previously (Journal of Rational Mechanics and Analysis, in press). Since explicit expressions of the coefficients of the above ordinary differential equation are obtained, relations between properties of the coefficients of (1) and those of the ordinary differential equation can be derived. (Received June 28, 1956.) 
682t. Erwin Kreyszig: On singularities of partial differential equations with analytic coefficients.

The equation (1) $\Delta \phi+\alpha\left(z_{1}, z_{2}\right) \phi_{z^{1}}+\beta\left(z_{1}, z_{2}\right) \phi_{z^{2}}+\gamma\left(z_{1}, z_{2}\right) \phi=0$ can be transformed into (2) $u_{z z}++B\left(z, z^{+}\right) u_{z^{+}}+C\left(z, z^{+}\right) u=0$ where $z=z_{1}+i z_{2}, z^{+}=z_{1}-i z_{2}$ are independent variables if $z_{1}$ and $z_{2}$ are complex. Let $B, C$ be such that the functions $(3) u\left(z, z^{*}\right)$ $=\int_{-1}^{1} \exp \left(\sum_{\alpha_{-1}}^{m} q_{\alpha}\left(z, z^{*}\right) t^{\alpha}\right)\left(z\left(1-t^{2}\right) / 2-\beta\right)^{-n}\left(1-t^{2}\right)^{-1 / 2} d t$ are solutions of $(2)$. Then $u\left(z, z^{*}\right) \equiv U\left(z_{1}, z_{2}\right)$, considered as a function of $z_{1}$, satisfies an ordinary linear differential equation (4) $\sum_{\rho_{m 0}}^{k} G_{\rho}\left(z_{1}, z_{2}\right)\left(d^{\rho} U / d z_{1}^{\rho}\right)=0$ of degree $k$ not exceeding $m+3$; in (4), $G_{\rho}\left(z_{1}, z_{2}\right) \equiv g_{\rho}\left(z, z^{*}\right)$. Theorems: I. If $B$, considered as a function of $z$ for any finite $z^{*}=z_{0}^{*}=$ const., has a pole of $r$ th order at a point $z=a$, then $g_{\rho}$, considered as a function of $z$, has at $z=a$ a pole of order $w(m, \rho) r$ where $w(m, \rho)=m-\rho+3$. In particular, if $r=1$ then (4) is of Fuchs' type at $z=a$. II. If $q_{1} \not \equiv 0$, cf. (3), and $C$, considered as a function of $z^{*}$ for any finite $z=$ const., has a pole of order $2 s-1, s>1$, at a point $z^{*}=a^{*}$ then $g_{\rho}$, considered as a function of $z^{*}$, has at $z^{*}=a^{*}$ a pole of order $w(m, \rho) s-\left(\delta_{1 \rho}\right.$ $\left.+\delta_{3 \rho}\right)(s-1)$ (if $m=1$ ) or $w(m, \rho)+\sum_{n=0}^{\{(m+2) / 21} \delta_{2 n, \rho}(s-1)$ (if $\left.m>1\right)$, where $\delta_{i k}$ is the Kronecker symbol. Using these theorems the behavior of the above solutions $u\left(z, z^{*}\right)$ in the neighborhood of the singularities of (2) can be investigated by means of the theory of ordinary differential equations. (Received June 28, 1956.)

683. Stephen Kulik: A note on the Laguerre method for separating the roots of an algebraic equation.

Let $f(x)=0$ be an algebraic equation with all its roots real and let $D_{n}$ be a determinant of order $n$, the $k$ th line of which is $\mid f_{x}^{(k)} /(k-1) ! f^{(k-1)} /(k-1) ! f^{(k-2)} /(k-2)$ ! $\cdots f(x) \cdots 0 \mid$. Any two numbers $u$ and $v$, satisfying the equation $(u-x)(v-x) D_{n}$ $+(u+v-2 x) f(x) D_{n-1}+f^{2}(x) D_{n-2}=0$ with $n$ even and $x$ an arbitrary real number, separate the roots of $f(x)=0$. If $x$ is closer to a root $a$ than to any other root of the equation and $v$ is fixed, then $u$ converges to $a$ as $n \rightarrow \infty$. If $v$ and $x$ are not separated by any root of $f(x)=0$, then $u$ and $x$ will be separated by the root a starting with some definite value of $n$. (Received June 11,1956.)

684t. R. A. Kunze: The Hausdorff-Young theorem for locally compact unimodular groups. Preliminary report.

Let $\Gamma=(\mathcal{H}, Q, m)$ be a gage space determined by a ring of operators $Q$ on a Hilbert space $\mathcal{H}$, and a regular gage $m$ on the projections in $Q$. A measurable operator $T$ has for $1 \leqq p \leqq \infty$ a norm $\|T\|_{p}$, and the collection $L_{p}(\Gamma)$ of all measurable operators with $\|T\|_{p}$ finite is a Banach space. A locally compact unimodular group $G$ determines a gage space $\Gamma(G)=\left(L_{2}(G), \mathscr{L}, m\right)$ where $\mathcal{L}$ is the ring generated by left translations on $L_{2}(G)$ by elements of $G$. By an extension of the Riesz-Thorin interpolation theorem the following Hausdorff-Young theorem is obtained. For $1 \leqq p \leqq 2$ and $f \in L_{2}(G)$ the operator $L_{f}$ of convolution by $f$ in $L_{2}(G)$ is measurable and $\left\|L_{f}\right\|_{p^{\prime}} \leqq\|f\|_{p}$ where $1 / p+1 / p^{\prime}=1$. For compact groups the above global result can be stated as follows: Suppose $G$ has Haar measure 1 and let $\left\{\phi_{\lambda}\right\}$ be any collection of inequivalent continuous irreducible unitary representations of $G$ where $\phi_{\lambda}$ has degree $d_{\lambda}$. Put $F_{\lambda}$ $=\int_{G} f(a) \phi_{\lambda}(a) d a$ and let $U_{\lambda}\left|F_{\lambda}\right|$ be the canonical polar decomposition of $F_{\lambda}$. Then $\left(\sum_{\lambda}\left(\text { trace }\left|F_{\lambda}\right| p^{\prime}\right) d_{\lambda}\right)^{1 / p^{\prime}} \leqq\|f\|_{p}$. (Received April 26, 1956.)

685t. J. G. de Lamadrid: On a function space. Preliminary report.

Let $\Lambda^{2}$ be the vector space of all real valued functions $f$ defined on the whole finite 
real axis, such that the Lebesgue integral $p_{a}^{b}(f)_{b}^{2}=\int_{a}^{b} f^{2}(x) d x<+\infty$ exists for every finite interval $[a, b] . p_{a}^{b}$ is a semi-norm of $\Lambda^{2}$ and the system $\left\{p_{a}^{b}\right\}$ defines a locally convex linear topology of $\Lambda^{2}$. The purpose of this note is to study the topology and geometry of $\Lambda^{2}$. Let $H_{i}$ be the Hilbert space of all functions of $\Lambda^{2}$ vanishing outside of $[-i, i] i=1,2,3, \cdots$, with the inner product defined in an obvious way. Clearly, $H_{1} \subset H_{2} \subset H_{3} \ldots$ It is shown that $\Lambda^{2}$ is the projective limit (Dieudonne, Bull. Amer. Math. Soc. vol. 59 (1953) p. 502) of the sequence $\left\{T_{i}, H_{i}\right\}$, where $T_{i} f=e_{i} f, e_{i}$ is the characteristic function of $[-i, i]$ and $\left(e_{i} f\right)(x)=e_{i}(x) f(x)$. From this and other properties it follows that $\Lambda^{2}$ is an $\mathcal{F}$-space (metrizable and complete), non-normable, and reflexive. Its dual space is found to be $\bigcup_{i=1}^{\infty} H_{i}$, the space of functions in $\Lambda^{2}$, each of which vanishes outside some finite interval, and that the dual with its strong topology is the strict inductive limit of the sequence $H_{i} C H_{2} C H_{3} \subset \cdots$ These results are generalized from $\Lambda^{2}$ to a class of spaces defined in the paper and termed monotone projective limits of Hilbert spaces. (Received July 6, 1956.)

\section{6t. P. D. Lax: An abstract stability theorem, II.}

Let $H$ be a Hilbert space and $D$ an unbounded operator with the following property: there exists an infinity of lines parallel to the imaginary axis with arbitrarily large negative real parts on which the resolvent of $D$ is uniformly bounded by some constant $d^{-1}$. For $D$ selfadjoint this is equivalent to the presence of infinitely many gaps of length $2 d$ in the spectrum of $D$ on the negative axis. Let $u(t)$ be a solution of $u_{t}=(D+K(t)) u,\|K(t)\| \leqq k<d$. Conclusion: unless identically zero, $u(t)$ tends to zero no faster than some exponential. The restriction on the constant $k$ is the mildest possible. This result is used in studying the local behavior of solutions of elliptic equations. (Received June 4, 1956.)

\section{7t. Karel deLeeuw: Almost periodic functions on the half-line.}

Let $B$ be the space of all bounded continuous functions on the half-line $\{x: x$ real and $x \geqq 0\}$. For each $f$ in $B$ and each real $x \geqq 0$, define $x f$ in $B$ by $x f(y)=f(y+x)$. Let $A$ be the subspace of $B$ that consists of all those $f$ with the set of all ${ }_{x} f$ totally bounded (that is, having compact closure) in $B$ supplied with the topology induced by the norm $\|h\|=\operatorname{Sup}|h(x)|$. Let $A_{p}$ be the subspace of $A$ that consists of all functions that are the restriction to the half-line of continuous almost periodic functions on the entire line. Let $A_{\infty}$ be the subspace of $A$ that consists of all functions in $B$ that are zero at infinity. Theorem: $A$ is algebraically and topologically the direct sum of $A_{p}$ and $A_{\omega}$. A similar decomposition is obtained for a large class of sub-semigroups of abelian groups, the first factor being as above restrictions of almost periodic functions on the entire group, but the second factor is not easily identified. (Received June 25, 1956.)

688t. J. J. Levin: The asymptotic behavior of the stable initial manifolds of a system of nonlinear differential equations.

It is shown that under suitable hypothesis the stable initial manifolds associated with the system of differential equations (1) $d x / d t=f(t, x, y, \epsilon), \epsilon d y / d t=g(t, x, y, \epsilon)$ approach, as $\epsilon \rightarrow 0+$, the stable initial manifold associated with the system of differential equations (2) $d y / d \gamma=g(0, x(0), y, 0)$. The variables $x$ and $y$ are vectors of $m$ and $n$ components respectively; (2) is the boundary layer equation for (1). It is also shown that the solutions of (1) are very well approximated for small $t$ and $\epsilon$ by corresponding solutions of (2). (Received July $3,1956$. 
689t. W. S. Loud: Some growth theorems for linear ordinary differential equations.

Liapounoff (Problème général de la stabilité du mouvement, Princeton, 1949) has shown that if $A(t)$ is a matrix of real, piecewise continuous, bounded functions for all $t \geqq 0$, there exists a finite real number $\lambda_{0}$ such that if $\lambda>\lambda_{0}$, and $x(t)$ is any nontrivial vector solution of $x^{\prime}=A(t) x$, then $x(t) e^{-\lambda_{t}} \rightarrow 0$ as $t \rightarrow \infty$. Here it is shown that if $A(t)$ $=\left\{a_{i j}(t)\right\} i, j=1,2, \cdots, m$, and if $\sum \sum\left|a_{i j}(t)\right| \leqq A^{n}(A>0)$ for all $t \geqq 0$, then $\lambda_{0} \leqq A$ for the system $x^{(n)}=A(t) x$. If in $x^{\prime \prime}+a(t) x=0, a(t)$ is real and piecewise continuous, and $\alpha \leqq a(t) \leqq \beta$ for all $t \geqq 0$, then $\lambda_{0} \leqq(-\alpha)^{1 / 2}$ if $3 \alpha+\beta \leqq 0$, and $\lambda_{0} \leqq 2^{-3 / 2}(\beta$ $-\alpha)(\beta+\alpha)^{-1 / 2}$ if $3 \alpha+\beta \geqq 0$. If in $x^{\prime \prime}+c x^{\prime}+a(t) x=0, a(t)$ is as above and $\alpha>0$, then for any solution, $x(t)$ and $x^{\prime}(t)$ approach zero exponentially if $c>\beta^{1 / 2}-\alpha^{1 / 2}$. This work was supported in part by the Office of Ordnance Research. (Received June 15, 1956.)

\section{0t. W. S. Loud: Behavior of certain forced nonlinear systems with large forcing.}

In the equation $\left(^{*}\right) x^{\prime \prime}+[c+f(x)] x^{\prime}+k x+g(x)=A e(t)$, let the following hypotheses be satisfied. (1) $e(t)$ has period $L$; (2) $c$ and $k$ are constants such that $x^{\prime \prime}+c x^{\prime}+k x=0$ has no solution of period $L ;(3) g(x)$ is bounded and satisfies a Lipschitz condition for which the constant approaches zero for large $|x|$; (4) $f(x)$ and $F(x)=\int_{0}^{x} f(u) d u$ are bounded, and $f(x) \rightarrow 0$ as $|x| \rightarrow \infty$; (5) the unique solution $\phi_{0}(t)$ of period $L$ of $x^{\prime \prime}+c x^{\prime}+k x=e(t)$ is not zero in any interval. Then if $A$ is large enough, $\left(^{*}\right)$ has a unique solution $x_{0}(t)$ of period $L$ such that $\left|A^{-1} x_{0}(t)-\phi_{0}(t)\right|=O\left(A^{-1}\right)$. A similar result holds when $k=0$ under certain additional hypotheses. This generalizes a result of the author for systems with limiting (Bull. Amer. Math. Soc. Abstract 62-1-102). If a proper subharmonic of order $n$ for $\left(^{*}\right)$ is a solution having $n L$ but no smaller multiple of $L$ as a period, the result shows that for any particular $n$ there are no proper subharmonics of order $n$ for large enough $A$. The proofs are based on equivalent integral equations and use the near linearity of $\left(^{*}\right)$ for large $|x|$. This work was supported in part by the Office of Ordnance Research. (Received June 25, 1956.)

691. E. B. McLeod, Jr.: The solution of an extremal problem in the complex domain by the variation of Schiffer.

It is a well-known classical result that within a family of domains, each of which has a fixed outer mapping radius and is bounded by a closed rectifiable curve, the one which has a boundary of minimum length is a circle. A generalization of this problem is to impose the constraint that the complement of each of these competing contains a fixed linear segment. Once the existence of an extremal domain has been established, a method of comparison devised by M. Schiffer may be used. This device of Schiffer has the advantage that only interior points of the domain are involved and no hypothesis which concerns the smoothness of the boundary is necessary. The analyticity of the boundary is deducible by this method. The use of the Schiffer Variation leads us to a quadrature representation of the analytic function which maps the exterior of the unit circle onto the extremal domain. (Received May 21, 1956.)

\section{2t. T. S. Motzkin and J. L. Walsh: Infrapolynomials.}

Let the compact set $E$ contain if finite $m>n>0$ points. A polynomial $f(z)$ $\equiv z^{n}+\cdots$ is an infrapolynomial on $E$ if no $g(z) \equiv z^{n}+\cdots$ exists with $|g(z)|<|f(z)|$ on $E$ where $f(z) \neq 0, g(z)=f(z)$ on $E$ where $f(z)=0$; an equivalent condition is that no $g(z) \not \equiv f(z)$ exists with $|g(z)| \leqq|f(z)|$ on $E$. The class $F_{n}$ of such $f(z)$ is closed and 
connected, but convex for $n=1$ or $m-1$ only. Any $f(z)$ in $F_{n}$ is the limit of infrapoly* nomials on finite subsets of $E$. A polynomial $f(z)$ is in $F_{n}$ if and only if $f(z) \equiv g(z) h(z)$, where $g(z)$ has only, and the infrapolynomial $h(z)$ on $E$ has no, zeros of the third kind (i.e., at limit points of $E$ ); also, if and only if $f(z) \equiv g(z) h(z)$ where $g(z)$ (necessarily in some $F_{v}$ ) has only zeros of the third kind and $h(z)$ is a factor of some infrapolynomial $\sum_{1}^{r+1} \lambda_{i \omega}(z) /\left(z-z_{i}\right)$ on $\left\{z_{1}, \cdots, z_{r+1}\right\}$, with $\omega(z) \equiv \prod_{1}^{r+1}\left(z-z_{i}\right), \lambda_{i} \geqq 0, \sum \lambda_{i}=1, z_{i}$ on $E$. If $E$ is real, a necessary and sufficient condition that $f(z)$ be in $F_{n}$ is that the $\mu$ zeros not of third kind of $f(z)$ separate $\mu+1$ distinct points of $E$. (Received July 6 , 1956.)

\section{3t. J. C. C. Nitsche: On the solutions of the differential equation} $r t-s^{2}=1$.

Author presents an elementary proof of the following theorem (c.f. K. Joergens, Math. Ann. vol. 127 (1954): Let the function $z(x, y)$ be of class $C^{2}$ and satisfy $r t-s^{2}$ $=1, r>0$ for all values of $x$ and $y$. Then $z(x, y)$ is a quadratic polynomial. This proof is based on the facts: (i) The transformation $\xi=x+p(x, y), \eta=y+q(x, y)$ is a one-toone mapping of the $x, y$-plane onto the $\bar{\xi}_{1} \eta$-plane (cf. H. Lewy, Trans. Amer. Math. Soc. vol. $41(1937))$. (ii) The function $f(\sigma)=(x-p)-i(y-q)$ is a regular analytic function of the variable $\sigma=\xi+i \eta$. (iii) $f^{\prime}(\sigma)=[(t-r)+2 i s] \cdot(2+r+t)^{-1}$ and $\left|f^{\prime}(\sigma)\right|<1$. Thus $f^{\prime}(\sigma)$ must be a constant and hence $r=$ const., $s=$ const., $t=$ const. An immediate consequence of the proved theorem (cf. a remark of E. Heinz in the quoted paper by Joergens) is the famous theorem by S. Bernstein: A minimal surface which can be represented in the form $z=z(x, y)$ where $z(x, y)$ is of class $C^{2}$, for all $x, y$ must be a plane. Applying Schwarz's lemma to the function $f(\sigma)$ one can derive bounds in terms of the second order derivatives for the third and higher order derivatives of a solution $z(x, y)$ of $r t-s^{2}=1$. Also further results concerning the behaviour of $z(x, y)$ can be obtained. (Received June 18, 1956.)

694t. J. C. C. Nitsche: An analytic function connected with the minimal surface equation and Bernstein's theorem.

Let the function $z=z(x, y)$ be twice continuously differentiable and satisfy the minimal surface equation in a domain $G$ of the $x, y$-plane. Consider the transformation $\xi=x+\int_{\left(x_{0}, y_{0}\right)}^{(x, y)} W^{-1}\left[\left(1+p^{2}\right) d x+p q d y\right], \quad \eta=y+\int_{\left(x_{0}, y_{0}\right)}^{(x, y)} W^{-1}\left[p q d x+\left(1+q^{2}\right) d y\right]$. Here $W$ $=\left(1+p^{2}+q^{2}\right)^{1 / 2}$. Because of $\left(\xi_{2}-\xi_{1}\right)^{2}+\left(\eta_{2}-\eta_{1}\right)^{2} \geqq\left(x_{2}-x_{1}\right)^{2}+\left(y_{2}-y_{1}\right)^{2}$ this is a one-toone mapping of $G$ onto a domain $\Gamma$ in the $\xi, \eta$-plane. If $z(x, y)$ is defined in the whole $x, y$-plane then $\Gamma$ coincides with the whole $\xi, \eta$-plane. Now observe that the function $F(\sigma)=(q+i p)(1+W)^{-1}$ is a regular analytic function of the variable $\sigma=\xi+i \eta$, defined in $\Gamma$. One has the inequality $|F(\sigma)|<1$. Thus, if $z(x, y)$ is an "entire" solution, that is if $G$ coincides with the whole $x, y$-plane then $F(\sigma)$ must be a constant. Hence $p=$ const. and $q=$ const. and hence $z=a+b x+c y$. This proves Bernstein's theorem on minimal surfaces. Using Schwarz's lemma, the fact that $|F(\sigma)|<1$ in $\Gamma$, and the "shrinking property" of the mapping $\xi, \eta \rightarrow x, y$ one can infer bounds in terms of the first order derivatives for the second and higher order derivatives of $z(x, y)$ and further results. (Received June 25, 1956.)

695t. J. C. C. Nitsche: On the solutions of the differential equation $\Delta \phi=\exp \phi$.

Suppose $\phi(z, \bar{z})$ is a real solution of $\left(^{*}\right) \Delta \phi=\exp (2 \phi)$, twice continuously differentiable in a domain $G$. Then the function $w(z)=\phi_{z z}-\phi_{z}^{2}$ is a regular analytic function of $z$ 
defined in $G$ (see L. Bieberbach, Math. Ann. vol. 77). Conversely, given a regular analytic function $w(z)$ and the initial conditions $\phi=\log 2 \alpha, \phi_{z}=\beta+i \gamma$ in $P_{0}\left[z=z_{0}\right.$, $\left.\bar{z}=\bar{z}_{0}\right]$, the corresponding solution $\phi$ of $\left(^{*}\right)$ can be determined in the following way: Let $\psi^{1}(z)$ and $\psi^{2}(z)$ be a fundamental system of $\left(_{*}^{*}\right) \psi_{z z}+w \psi=0$ satisfying $\psi^{1}=1$, $\psi_{z}^{1}=-(\beta+i \gamma) ; \psi^{2}=0, \psi_{z}^{2}=1$ in $P_{0}$. Then $\phi=\log 2 \alpha-\log Q$ where $Q=\left|\psi^{1}(z)\right|^{2}-\alpha^{2}$ $\left|\psi^{2}(z)\right|^{2}=\left|\psi^{1}(z)\right|^{2} \cdot\left\{1-\alpha^{2}\left|\int_{z_{0}}^{z}\left[\psi^{1}(u)\right]^{-2} d u\right|^{2}\right\}$. From this representation a proof for the nonexistence of "entire" solutions of $\left(^{*}\right)$ follows (for another proof see Wittich, Math. Zeit. vol. 49): Otherwise $w(z)$ and $\psi^{k}(z)$ would also be entire functions of $z$. Now $Q=1$ in $P_{0}$ and $Q$ also must assume negative values. Because if everywhere the inequalities $\left|\psi^{1}\right|^{0}>0$ and $Q>0$ would hold then the integral in the brackets would be bounded which is a contradiction due to Liouville's theorem. If the point $P_{1}\left[z=z_{1}\right.$, $\left.\bar{z}=\bar{z}_{1}\right]$ is an isolated singularity of $\phi$, and if $\phi_{z}$ is single valued in a neighborhood of $P_{1}$, and if $\left(\begin{array}{c}* \\ *\end{array}\right)$ has a regular singularity there then this singularity of $\phi$ is of either one of the two types $c \cdot \log \left|z-z_{1}\right|$ or $c \cdot \log |\log | z-z_{1}||$. (Received July 3, 1956.)

696t. J. C. C. Nitsche: On harmonic mappings and a lemma of Rado-Bers.

Let $x=\operatorname{Re} F(\zeta), y=\operatorname{Re} G(\zeta), \zeta=\xi+i \eta$ be a one-to-one harmonic mapping of the unit disc $\Gamma[|\zeta|<1]$ onto a domain $C$ of the $x, y$-plane. T. Rad6 (Math. Zeit. vol. 26) and L. Bers (Annals of Math. (2) vol. 53) have proved that $C$ cannot coincide with the whole $x, y$-plane. This can easily be shown using the theorem that an entire solution of the differential equation $r t-s^{2}=1$ must be a quadratic polynomial (for a proof which makes no use of the properties of harmonic mappings see a preceding abstract of the author). According to a theorem of H. Lewy (Bull. Amer. Math. Soc. vol. 42) $\operatorname{Im}\left(F^{\prime} \bar{G}^{\prime}\right)=x_{\xi} y_{\eta}-x_{\eta} y_{\xi} \neq 0$ in $\Gamma$. Form the expressions (see K. Joergens, Math. Ann. vol. 129, Lemma 3) $r=\left|G^{\prime}\right|^{2} \cdot\left[\operatorname{Im} F^{\prime} \bar{G}^{\prime}\right]^{-1}, s=-\left[\operatorname{Re} F^{\prime} \bar{G}^{\prime}\right] \cdot\left[\operatorname{Im} F^{\prime} \bar{G}^{\prime}\right]^{-1}, t=\left|F^{\prime}\right|^{2}$ - $\left[\operatorname{Im} F^{\prime} \bar{G}^{\prime}\right]^{-1}$ and consider $r, s, t$ as functions of $x$ and $y$ defined in $C$. Because of $r_{y}-s_{x}=s_{y}-t_{x}=0$ there exists a function $z(x, y)$ such that $r=z_{x x}, s=z_{x y}, t=z_{y y}$ and $z_{x x} z_{y y}-z_{x y}^{2}=1$ in $C$. Now assume that $C$ is the whole $x, y$-plane. Then $r, s, t$ must be constants. Thus the equation $F(\zeta)=(\alpha+i \beta) G(\zeta)+(\gamma+i \delta)$ with real constants $\alpha$, $\beta \neq 0, \gamma, \delta$ would hold true. After the linear transformation $u=y, v=(\gamma-x) \beta+y \alpha / \beta$ one then gets $w=u+i v=G(\zeta)$. This is a contradiction. (Received July 16,1956.)

\section{7t. Robert Osserman: On the solution of a functional equation.}

In answer to a problem posed by $\mathrm{S}$. Chowla on the existence of an entire solution of $f(f(z))=e z-1$, the following theorem is proved. Let $S$ be a strip $-b<\operatorname{Im}\{z\}<b$ for some $b>\pi$. Let $f(z)$ be a function defined in a domain $D$ such that $S \subset D$ and $f(S) \subset D$. If $f(z)$ satisfies the equation $f(f(z))=e^{x}-1$ throughout $D$ then $f(z)$ cannot be analytic throughout $S$. Both Thron (Canadian Journal of Mathematics vol. 8 (1956) pp. 4748) and Baker (Math. Ann. vol. 129 (1955) pp. 174-180) have proved that there exist no entire solutions of $f(f(z))=F(z)$ for a wide class of functions $F(z)$ including $F(z)$ $=e z-1$ and $F(z)=e z$. The latter case was investigated by Kneser (J. Reine Angew. Math. vol. 187 (1950) pp. 56-67) who showed that there exists a solution analytic on the whole real axis. Our proof also applies to show that Kneser's solution cannot be extended analytically throughout $S$. (Received July 23, 1956.)

698. O. O. Pardee: On representations for certain variational problems. Preliminary report.

On sets of functions closed in $L_{2}$ whose derivatives or integrals are also closed and 
which satisfy certain auxiliary conditions suitable for use in an explicit representation of J. Indritz (Pac. J. Math. vol. 5 (1955) pp. 765-797) for the function $u^{*}$ (assumed) which minimizes the functional $I(u)=\iint_{R}\left(a u_{x}^{2}+b u_{y}^{2}+c u^{2}\right) d x d y$. Here $R$ is a plane bounded region with a smooth boundary curve $C$; the functions $a, b, c$ are bounded, integrable on $R+C$ and $a>0, b>0, c \geqq 0$. The minimal function is represented by $u^{*}=g(x, y)+\sum f_{k}(x, y)$ where $g(x, y)$ is a continuously differentiable function defined on $R+C$ attaining prescribed boundary values on $C$, and the functions $f_{k}(x, y)$ are defined as follows: Let $G(x, y)$ be a function continuously differentiable on $R+C$, positive in $R$, vanishing on $C$, with positive normal derivative on $C$, and let $P_{k}(x, y)$ be a dense set of polynomials defined over a region containing $R+C$. Then $f_{k}(x, y)$ are the functions obtained by orthonormalizing the set $\left(G P_{k}\right)$ where the square of the norm is $I(u)$. The present paper determines more general sets of functions than polynomials for the functions $P_{k}$ and removes some additional smoothness conditions required on the boundary $C$ and the functions $g$ and $G$. (Received July 2,1956.)

699. R. N. Pederson: A new inequality for the unique continuation theorem. Preliminary report.

Let $\hat{A}=a^{i i}(x) \partial^{2} / \partial x_{i} \partial x_{j}+b^{i}(x) \partial / \partial x_{i}+c(x)$ be an elliptic operator defined in a neighborhood of the origin. Let $A$ be the Laplace-Beltrami operator corresponding to the metric $d s^{2}=a_{i j} d x_{i} d x_{j}$, where $\left(a_{i j}\right)$ is the inverse matrix of $\left(a^{i j}\right)$. Let $r$ be the geodesic distance from the origin. We prove that there exist constants $R_{0}, \alpha_{0}$ such that $\int \exp (\alpha / r)(A u)^{2} d V \geqq \alpha \int \exp (\alpha / r)\left(\triangle u \cdot \triangle u+u^{2}\right) d V$ for $R \leqq R_{0}, \alpha \geqq \alpha_{0}$. Here $d V$ denotes the volume element induced by the metric, the integration is performed over the geodesic sphere $r \leqq R$, and $u \in C^{2}$ is assumed to vanish both near zero and near $R$. The proof follows easily after substituting $u=r^{p} \exp (-\alpha / r) v$ with $p=(6-n) / 2$. The unique continuation theorem for the operator $\hat{A}$ can be shown to be a consequence of the above inequality by an argument similar to the one employed by Heinz (Nachr. Ges. Wiss. Gottingen (1955) pp. 1-12) and Aronszajn (Comptes rendus, vol. 242 (1956) pp. 723-725). (Received June 12, 1956.)

700. F. W. Perkins: On the Almansi-Nicolesco theorem. Preliminary report.

The Almansi-Nicolesco Theorem asserts that if $u_{p}$ is $p$-harmonic (that is, if $\Delta^{p} u_{p} \equiv 0$ ) in a domain $D$ and if $M$ is a fixed point of $D$ then (in a certain subdomain of D) $u_{p}=V_{0}+V_{1} r^{2}+V_{2} r^{4}+\cdots+V_{p-1} r^{2(p-1)}$ where $r$ is the distance from $M$ and $V_{0}, V_{1}, \cdots, V_{p-1}$ are harmonic functions uniquely determined by $u_{p}$ and $M$. (M. Nicolesco, Ann. École Norm. vol. 52 (1935) pp. 183-220; see particularly pp. 182192.) The present paper contains a discussion of a number of topics connected with this theorem, including an alternative method of determining the functions $V_{0}$, $V_{1}, \cdots, V_{p-1}$, once their existence is known. (Received July $\left.3,1956.\right)$

\section{R. S. Phillips: Dissipative hyperbolic systems.}

This paper is concerned with the Cauchy problem for hyperbolic systems in only one spatial variable of the form $E y_{t}=(A y)_{x}+B y, a<x<b, t>0$; here $y$ is a vectorvalued function of $(x, t)$ and $E, A, B$ are matrix-valued functions of $x$ alone, $E$ being hermetian positive definite, $A$ hermetian of constant rank, and $B$ satisfying the condition $B+B^{*}+A_{x} \leqq \Theta$ for all $x \in(a, b)$. It is assumed that $E$ and $A$ are absolutely continuous on each compact subinterval of $(a, b)$, and that the elements of $E_{x}, A_{x}$, and $B$ are square integrable on each compact subinterval of $(a, b)$. By applying methods 
from the theory of semi-groups all possible semi-group solutions are found for which $(A y, y)^{b}-(A y, y)^{a} \leqq 0$ for all functions in the domain of the generator: here the base space is the hilbert space with norm $\left[\int_{a}^{b}(E y, y) d x\right]^{3 / 2}$. In order to include all of the "dissipative" boundary conditions commonly associated with hyperbolic systems, the above differential system has been coupled at the end points to finite degree of freedom dissipative systems and all possible semi-group solutions found, again subject to the condition that no energy enters through the boundary. (Received July 13, 1956.)

\section{R. T. Prosser: $C^{*}$ algebras with trace.}

This paper presents a systematic study of those $C^{*}$ algebras which admit a densely-defined faithful effective trace. Such algebras admit an abstract integration theory which is entirely analogous to that of the commutative case. Moreover, every such algebra is strongly semi-simple, and there is a natural one-one correspondence between the set of maximal two-sided ideals, and (modulo a scalar factor) the set of extreme traces. These extreme traces may be densely embedded in a certain locally compact Hausdorff space over which the algebra may be decomposed into factors which are almost all simple. An analysis of this decomposition shows that there are essentially two types of these algebras: The CCR algebras of type I and the FR algebras of type II. (Received July 6, 1956.)

\section{3t. M. H. Protter: Uniqueness theorem for the Tricomi problem.}

Let $K(y)$ be a monotone increasing twice continuously differentiable function with $K(o)=0$. Let $D$ be a domain bounded by an arc $\Gamma_{1}$ lying in $y>o$ with end points on the $x$-axis at $A$ and $B$ and by the characteristics $\Gamma_{2}$ and $\Gamma_{3}$ issuing from $A$ and $B$ which intersect. Theorem: Any solution of $K(y) U_{x x}+U_{y y}=0$ which vanishes on $\Gamma_{1}$ and on one of the characteristics vanishes throughout $D$ provided that $K^{\prime}(y)>0$ for $y<0$. The condition $K^{\prime}(y)>0$ may be weakened by assuming that $\left(3 K^{\prime 2}-2 K K^{\prime \prime}\right) / K^{\prime 2} \neq-\infty$ for $y<0$. This contains as special cases the previous results of Frankl, Ou, Ding, and the author. (Received May 4, 1956.)

\section{Edgar Reich: On schlicht functions with real coefficients. II.}

Let $S_{\mathrm{r}}$ be the class of functions $f(z)=z+a_{2} z^{2}+\cdots\left(a_{k}\right.$ real $)$ which are regular and schlicht for $|z|<1$ (Cf. Bull. Amer. Math. Soc. Abstract 61-5-613). By transforming the Basilewitsch-Tammi equation qualitative results regarding $m\left(z_{0}\right)=\inf f\left(z_{0}\right), f \in S_{r}$, in the "unknown" region $\left|R\left(z_{0}+1 / z_{0}\right)\right| \leqq 2$ are obtained; e.g., $m(z)$ has an absolute maximum at an interior point of $|z|<1$. Let $s(\alpha)$ be the class of functions $w=h(z)$ $=a_{1} z+a_{2} z^{2}+\cdots\left(a_{k}\right.$ real $), 0<a_{1} \leqq \alpha,|h(z)|<1$, regular and schlicht for $|z|<1$. The variability region of $h(z)$ is the set of values $w$ with $|w|<1$, such that $w /\left(1+w^{2}\right)$ $=\beta z /\left(1+2 \gamma z+z^{2}\right)$, as $(\beta, \gamma)$ range over all real pairs with $0<\beta \leqq \alpha,|\gamma| \leqq 1-\beta$. (Received June 27, 1956.)

\section{5t. P. V. Reichelderfer: A covering theorem for transforms.}

Given a continuous transformation $T$ from a bounded domain in Euclidean $n$ space $R^{n}$ into a bounded portion of $R^{n}$. Assume that $T$ is essentially of bounded variation. Let $D$ be a family of domains $D$ for each of which $T D$ is a domain whose boundary has Lebesgue measure zero, $D$ is a component of $T^{-1} T D$, and there exists a point $x$ in $R^{n}$ such that the topological index of $x$ with respect to $T$ and $D$ is defined and not zero. Consider a nonempty set $U$ for each point $u$ of which there exists a sequence of 
domains $D_{j}$ in $D$ containing $u$ and such that their images $T D_{j}$ have parameters of regularity uniformly bounded away from zero and diameters tending to zero as $j$ becomes large. Then there exists a subset $U_{0}$ of $U$ such that the Lebesgue measure of $T U_{0}$ is zero and the set of points in $U$ and not in $U_{0}$ is covered by a countable sequence of pairwise disjoint domains in D. (Received March 12, 1956.)

706t. Walter Rudin: Continuous functions on compact spaces without perfect subsets.

Let $Q$ be a compact Hausdorff space which has no nonvacuous perfect subset (examples show that $Q$ may be uncountable) and let $C(Q)$ be the Banach algebra whose elements are the continuous complex-valued functions on $Q$. It is proved that every member of $C(Q)$ has an at most countable set of values; it follows that every closed subalgebra of $C(Q)$ is self-adjoint. Consequently, every subalgebra of $C(Q)$ which separates points on $Q$ is dense in $C(Q)$. This provides a marked contrast between the structure of $C(Q)$ and that of $C(X)$, where $X$ is a compact Hausdorff space which has a subset homeomorphic to the Cantor set (see Bull. Amer. Math. Soc. Abstract 61-4-486.) It is also proved that every regular Borel measure on $Q$ is concentrated on an at most countable set of points. (Received June 19, 1956.)

707t. Walter Rudin: Subalgebras of group algebras.

The group algebra $L(G)$ of the locally compact abelian group $G$ has as its elements the complex-valued Haar-integrable functions on $G$; multiplication in $L(G)$ is convolution. A subset $S$ of $L(G)$ is said to be separating if the Fourier transforms of the members of $S$ separate points on the dual group. It is shown that the maximal ideals of $L(G)$ are the only separating proper closed subalgebras of $L(G)$ if $G$ is compact. On the other hand, if $G$ is the real line, then there is a separating maximal closed subalgebra $A$ of $L(G)$ (not an ideal) and a bounded totally disconnected perfect set $P$ of real numbers, with the following property: if $f \in A$ and if its Fourier transform $F$ is not constant on $P$, then the set consisting of the numbers $F(y)(y \in P)$ has dimension at least 1 . This seems to be the first example of a maximal subalgebra of the group algebra of the real line which is not a maximal ideal and which is separating. A similar example can be constructed for the case in which $G$ is the additive group of all integers. (Received June 19, 1956.)

\section{8t. P. P. Saworotnow: Diagonalization of a complemented algebra.}

The following is a consequence of the spectral theorem and the result recently obtained by the author [Bull. Amer. Math. Soc. Abstract 61-5-614t]. Every simple semi-simple complemented algebra can be realized as an algebra of all functions $a(s, t) \in L^{2}(S \times S)$ such that $\int K(s)|a(s, t)|{ }^{2} d s d t<\infty$, where $S$ is some measure space and $K(s)$ some positive, measurable, function such that $K(s) \geqq 1$ for all $s \in S$. (Received March 29, 1956.)

709t. H. M. Schaerf: On the existence of linear functionals invariant under operators. Preliminary report.

Let $L$ be a real linear space and let $G$ be a set of mappings of $L$ into $L$ such that $g \in G, l \in L$ implies $g(\alpha l)=\alpha g l$ for every scalar $\alpha$. Let $J$ be an arbitrary subadditive positively homogeneous functional defined on $L$. Then $(L, G, J)$ is called perfectible if there exists an additive homogeneous function $I$ which is defined on $L$, invariant under $G$ and such that $-J(-l) \leqq I(l) \leqq J(l)$ for every $l \in L$. Theorem 1 . Let $(L, G)$ 
be the set of all $\sum_{i=1}^{n}\left(g_{i} l_{i}-l_{i}\right)$ with $g_{i} \in G, l_{i} \in L$. In order that $(L, G, J)$ be perfectible it is necessary and sufficient that we have $J(l) \geqq 0$ for every $l$ in $(L, G)$. Theorem 2 . If $G$ is an abelian semi-group such that we have $g\left(\alpha_{1} l_{1}+\alpha_{2} l_{2}\right)=\alpha_{1} g l_{1}+\alpha_{2} g l_{2}$ for all $g \in G, l_{i} \in L, \alpha_{i}$ real $(i=1,2)$ and if, for every $l \in L$, the set $E(l)$ of all $J(g l)$ with $g \in G$ is bounded, then $\left(L, G, J^{*}\right)$ is perfectible where $J^{*}(6)$ is the least upper bound of $E(l)$. Theorems 1 and 2 generalize results of $E$. Folner and the author, respectively. Some applications of Theorem 2 are stated in another abstract. (Received July 5, 1956.)

710t. H. M. Schaerf: On the existence of invariant measures. Preliminary report.

Let $(X, S, m)$ be a totally finite measure space and let $G$ be an abelian semi-group of measurability preserving mappings of $X$ into $X$. Then there exists a finitely additive measure $\mu$ on $S$ which is invariant under $G$ and such that, for every $E \in S, \mu(E)$ is between the bounds of the set of all numbers $m\left(g^{-1} E\right)$ with $g \in G$. Therefore, $\mu$ is countably additive under each of the following conditions: (a) For every decreasing sequence $\left\{E_{n}\right\} \subset S$ whose intersection is empty, we have $\lim _{n \rightarrow \infty}\left[\sup _{g} \in a m\left(g^{-1} E_{n}\right)\right]$ $=0$; (b) $m(E)=0$ implies $m\left(g^{-1} E\right)=0$ for all $g \in G$ and $m(E)>0$ implies that the set of all numbers $m\left(g^{-1} E\right)$ with $g \in G$ has a positive lower bound. In case (b), $\mu$ has the same null sets as $m$. These results follow from Theorem 2 of the preceding abstract. (Received July 5, 1956.)

\section{1t. I. E. Segal: Ergodic subgroups of the orthogonal group on a real Hilbert space.}

A subgroup of the orthogonal group on a real Hilbert space is ergodic with respect to the isotropic normal (resp. Clifford) distribution, in the sense that there exist no invariant square-integrable functionals (resp. operators), if it is any of the following: (a) non-compact, simple, and finite-dimensional; (b) irreducible; (c) n-parameter abelian with pure continuous spectrum. Similar results hold also for the direct product of the normal and Clifford distributions, showing the nonexistence of a true $S$-operator for certain quantum fields. (Received July 9, 1956.)

\section{2t. V. L. Shapiro: Localization on spheres.}

Let $\Omega$ be the unit $(k-1)$-dimensional sphere in Euclidean $k$-space, $k \geqq 3$, and let $S=\sum_{n=1}^{\infty} Y_{n}(x)$ be a series of surface spherical harmonics defined on $\Omega$ with $Y_{n}(x)$ $=O\left(n^{\beta}\right)$ uniformly for $x$ on $\Omega, \beta \geqq 0$. To $S$ can be associated a Riemann function, $F(x)=\sum_{n=1}^{\infty} Y_{n}(x)[n(n+p)]^{-w}$, where $w$ is the smallest integer greater than $(\beta+1) / 2$ and $p=k-2$. It is then shown in this paper that if $F(x)$ is of class $C^{2 w}$ on a domain $D$ of $\Omega, S$ is uniformly $(C, \alpha)$ summable, $\alpha>p+2 w$, to $\Delta^{w} F(x)$ in any domain $D_{1}$ whose closure is contained in the interior of $D$, where $\Delta^{w}=\Delta\left(\Delta^{w-1}\right)$ and $\Delta$ is the Laplace operator on $\Omega$. This result extends to spheres the localization theorems obtained by Riemann and Zygmund for the circle. (Received July 3, 1956.)

\section{L. S. Shapley: Deviation of nonadditive set functions.}

The departure from additivity of real-valued functions on a Boolean ring of sets $B$ is considered. A tree partition of a set $S \in B$ is a collection $\left\{S_{\alpha} \mid \alpha \in A\right\}, A$ a tree, with $S_{0}=S$ and $\left\{S_{\beta i}\right\}$ partitioning $S_{\beta}$, where $0=\operatorname{glb} A$ and the $\beta i$ are the immediate successors of $\beta$ in $A$. The deviation of $f$ on $S$ is defined $\Delta(f, S)=\sup \sum_{\beta}\left|f\left(S_{\beta}\right)-\sum_{i} f\left(S_{\beta i}\right)\right|$, where the supremum is taken over all finite tree partitions of $S$, and $\beta$ is restricted to 
be nonterminal in $A$. Every function of finite deviation can be decomposed into superadditive, subadditive, and additive parts: $f=f^{+}+f^{-}+f^{0}$. The first two are defined in terms of "upper" and "lower" deviation, in analogy with the upper and lower variation of a real function of bounded variation. The third may be defined $f^{0}(S)$ $=\lim \sum_{i} f\left(S_{i}\right)$, where the limit is taken over the directed set of finite partitions $\left\{S_{i}\right\}$ of $S$, ordered by refinement. If the functions $f$ for which $f^{0}$ is well-defined and everywhere finite are called integrable, then the functions of finite deviation may be characterized as the subspace of the linear space of integrable functions spanned by the superadditive functions. (Received July 6, 1956.)

\section{4t. I. M. Sheffer: Some theorems on asymptotic series.}

Let $J(\alpha, \beta)$ be the sector $\alpha<\arg z<\beta,|z|>0$. A class $\mathcal{F}$ of analytic functions is called an asymptotic family for the sector $J(\alpha, \beta)$ if every series $\sum A_{n} / z^{n}$ is the asymptotic series of at least one $F(z) \in \mathcal{F}$. Carleman showed that the class $\mathfrak{F}$ of functions analytic in $\alpha<\arg z<\beta,|z|>R$ is an asymptotic family. It is here shown that for $J(0,2 \pi)$ an asymptotic family is the class of all functions single-valued throughout the complex plane, whose only finite singularities consist of a denumerable number of simple poles on the positive real axis with no finite limit point, together with a possible singularity at $z=0$. A corresponding result holds for the sector $J(0,2 k \pi)$, taken on the Riemann surface for $w$ defined by $w^{k}=z$. (Received July 6,1956 .)

715t. Walter Strodt: Approximate factorization of the homogeneous linear ordinary differential operators.

Let $A=\sum_{j=0}^{n} a_{j}(x) D^{i}$, with the $a_{j}(x)$ in a logarithmic domain $E^{*}$ and $a_{n}(x)$ nontrivial, (cf. author's Memoirs of the Amer. Math. Soc. no. 13). ( $\left.V, w_{1}, w_{2}, \cdots, w_{n}\right)$ is called a principal factorization sequence for $A$ if (1) $V$ and $w_{j}$ are logarithmic monomials, (2) $A(V) \sim 1$, (3) $A=F_{n} F_{n-1} \cdots F_{1} V+\sum_{j=0}^{n} \delta_{j}(x) F_{j} F_{j-1} \cdots F_{1} V$, with $\delta_{j}<1$, and $F_{j}=1-\left(1 / w_{j}(x)\right) D$, and (4) $\left(V, w_{1}, \cdots, w_{n}\right)$ is minimal for (1) (2) (3) under lexicographic $\prec$. Every $A$ has a principal factorization sequence and all sequences are obtainable by algebraic methods. $V$ is the principal monomial of $A(y)-1$. Let $E^{*}$ and $V$ have logarithmic ranks $\leqq p$. With the transform of $A(y)$ under $y=V z$ written $\sum_{j=0}^{n} c_{j}(x)\left[x \log x \log \log x \cdots \log _{p} x D\right]^{i} z, w_{1}, \cdots, w_{n}$ are logarithmic monomials, (determined by a Newton polygon), to which the zeros $r_{1}(x), \cdots, r_{n}(x)$ of $\sum_{j=0}^{n} c_{j}(x)\left[x \log x \log \log x \cdots \log _{p} x\right]^{i} r^{j}$ are asymptotically equivalent, numbered so that $w_{j+1}$ is never $\left\langle w_{j}\right.$. Every $w_{j}$ is in the divergence class. The proof applies the principal monomial algorithm to the generalized Riccati equation for $A$, i.e. to the transform of $y^{-1} A(y)$ under $h=y^{-1} D y$. If all $w_{j}$ have nonexceptional indices, (3) leads to a determination of the principal solutions of $A(y)=g$, whenever $g \sim a$ logarithmic monomial. (Received June 11, 1956.)

\section{6t. Walter Strodt: Uniformly quasi-linear algebraic ordinary differential equations.}

Let $w_{1}(x), \cdots, w_{n}(x)$ be logarithmic monomials in the divergence class, with indices $\left(C_{1}, k_{1}, t_{1}\right), \cdots,\left(C_{n}, k_{n}, t_{n}\right)$. Let $F_{j}=1-\left(1 / w_{j}\right) D, G_{j}=F_{j} F_{j-1} \cdots F_{1}, \delta_{j}(x)<1$ in $\bar{F}(a, b),(j=0,1, \cdots, n)$. Let $Q\left(x, Y_{0}, \cdots, Y_{n}\right)$ be a polynomial in the $\mathrm{Y}_{i}$, each term being of degree $\geqq 2$, and each coefficient being $<1$ or $\approx 1$. Let $f<1$. If $P(x, y$, $\left.D y, \cdots, D^{n} y\right)=f(x)+G_{n} y+\sum_{j=0}^{n} \delta_{j}(x) G_{j} y+Q\left(x, G_{0} y, G_{1} y, \cdots, G_{n} y\right), P$ is called uniformly quasi-linear. If every index is nonexceptional, a complete description is obtained of those solutions of $P=0$ which are $\prec 1$. Let $s_{j}=t_{j}$ if $k_{j}=0, s_{j}=0$ if $k_{j}>0$. Let 
$h_{j}(\theta)=\cos \left(s_{j} \theta+\arg C_{j}\right)$. For every $u$ in $(a, b)$ there exists $\delta>0$ such that $P=0$ has a nonempty family $S^{*}$ of solutions $<1$ in $\bar{F}(u-\delta, u+\delta)$. The number of arbitrary constants in $S^{*}$ equals the number of $j$ having $h_{j}(u)<0$. Every element of $S^{*}$ has an extension which is $<1$ in $\bar{F}\left(u_{1}, u_{2}\right)$, where $u_{1}=\max \left\{a, \theta: \theta<u ; h_{j}(\theta)=0\right.$ and $h_{j}^{\prime}(\theta)<0$ for at least one $j\}$, and $u_{2}=\min \left\{b, \theta: \theta>u ; h_{j}(\theta)=0\right.$ and $h_{j}^{\prime}(\theta)>0$ for at least one $\left.j\right\}$. The proof uses successive approximations $F_{j+1} Y_{j, k+1}=Y_{i+1, k},(j=0,1, \cdots, n-1)$, $Y_{n, k+1}=-f(x)-\sum_{j=0}^{n} \delta_{j}(x) Y_{j k}-Q\left(x, Y_{0 k}, \cdots, Y_{n k}\right), \quad(k=0,1, \cdots)$ (Received June 11, 1956.)

717t. Walter Strodt: Asymptotically quasi-linear algebraic ordinary differential equations.

Let $P\left(x, y, D y, \cdots, D^{n} y\right)$ be a nonvanishing differential polynomial with coeffcients in a logarithmic domain $L^{*}$; let $P$ be nontrivially of order $n$. Let $M$ be a principal monomial of $P$. Let $E<1$. Let $R\left(x, z, D z, \cdots, D^{n} z\right)$ be the transform of $P$ under the substitution $y=M(1+E)+z . P$ is called asymptotically quasi-linear at $M$ if $M$ is simple, $P$ is asymptotically nonsingular at $M$, and the homogeneous linear part of $R$ has a principal factorization sequence $\left(V, w_{1}, \cdots, w_{n}\right)$ independent of $E$. In a certain precise sense, almost every $P$ is asymptotically quasi-linear at every principal monomial $M$, with every $w_{j}$ of nonexceptional index. Whenever this occurs, $P=0$ has at least one solution $\sim M$ in a suitable $C S$. Let $L^{*}$ and $M$ have logarithmic ranks $\leqq p$. Let $\theta=x \log x \log \log x \cdots \log _{p} x D$. The proof uses operators $H_{j},(j=0,1, \cdots, n)$, where $H_{j} Q$ is obtained from any differential polynomial $Q$, written in terms of $\theta$, by omitting every nonlinear term whose order in $\theta$ exceeds $j$. A finite sequence $Q_{0}, Q_{1}, \cdots, Q_{n}$ of differential polynomials is constructed, with $H_{j} Q_{j}$ uniformly quasi-linear, and $Q_{j}$ equivalent to $R$ under $z=a_{j}(x) Z+b_{j}(x)$, where $a_{j}(x)$ and $b_{j}(x)$ are constructed using solutions $\prec 1$ of $H_{j-1} Q_{j-1}=0$. (Received June 11,1956.)

\section{8t. Walter Strodt: On a problem of R. Bellman.}

R. Bellman (Bull. Amer. Math. Soc. vol. 61 (1955) p. 192) posed the following question: "Does the equation $u^{\prime}+u=\left(u^{\prime \prime}\right)^{2}$ have a solution which approaches zero like $e^{-t}$ as $t \rightarrow \infty$ for suitably small values of $u(0)$ and suitably chosen $u^{\prime}(0)$ ?" A partial answer to this question, and other information about the given equation $G$, can be found in the following way: Let the subsitution $t=\log x, u=C x^{-1}+y$, (where $C$ is any nonzero complex constant), carry $G$ into $P=0$. The principal monomial $M$ of $P$ is $-C^{2} x^{-2}$. Under $y=M+M z, P$ is transformed into a uniformly quasi-linear equation $Q=0$ for which a description of the solutions $<1$ is available. Each such solution yields a solution $\sim C e^{-t}$ of $G$. Among other results, for every $C, Q=0$ has a solution $\prec 1$ in $\bar{F}(-\delta,+\delta)$, for some $\delta>0$. Hence $G$ has a solution $u$ with $u \sim C e^{-t}$ in a complex neighborhood system of $+\infty$ consisting of horizontal half-strips $S_{\epsilon}=\{t:|\mathscr{I}(t)|<\delta-\epsilon$; $\left.\mathbb{R}(t)>A_{\epsilon}\right\},(0<\epsilon<\delta)$. A continuity argument now shows that for every sufficiently small nonzero real $I$ there is a solution $u$ with $u(0)=I$, and $u(t) e^{t \rightarrow K}$ for some nonzero real $K$. (Received June 11, 1956.)

719. Vikramaditya Singh: Some extremal problems in univalent functions.

Schiffer's method of interior variation has been adapted to symmetric univalent functions, bounded univalent functions and bounded symmetric univalent functions. In particular, the following theorems are proved: Theorem 1 . Let $S_{0}$ be the class of regular univalent functions $f(z)$ in $|z|<1$ of the form $f(z)=z+\sum_{n=2}^{\infty} a_{n} z^{n}$ and let $R$ be its subclass with real coefficients. Let $F\left(a_{2}, a_{3}, \cdots, a_{n}, \bar{a}_{2}, \cdots, \bar{a}_{n}\right)$ be a real- 
valued function of $(n-1)$ variables $a_{v}$ and their complex conjugates $\bar{a} v, v=2, \cdots, n$. Then the functions $f_{R}(z) \subset R$ and $f_{S}(z) \subset S$ which maximize $F\left(a_{2}, \cdots, a_{n}, \bar{a}_{2}, \cdots, \bar{a}_{n}\right)$ satisfy the same functional differential equation. Theorem 2 . Let $S$ be the family of bounded regular univalent functions $f(z)$ in $|z|<1$ such that $|f(z)|<1, f(0)=0$ and $f(\xi)=\omega,|\xi|<1$. Then the maximum and minimum values of $\left|f^{\prime}(\xi)\right|$ are given respectively by $f^{\prime}(\xi)=(\omega(1+|\xi|) / \xi(1 \mp|\xi|))((1 \mp|\omega|) /(1 \pm|\omega|))$. The boundaries of the extremal domains consist of the unit circumference together with the radial slits starting respectively at the points $\mp|\omega| / \bar{\omega}$. Theorem 3 . Let $S_{1}$ be the family of bounded symmetric univalent functions $f(z)$ in $|z|<1$ satisfying $|f(z)|<1, f(0)=0, f(\xi)=\omega$, $-1<\xi<1$. Then the functions which give the maximum and the minimum of $f(\eta)$, $-1<\eta<1$ are respectively given by $f(z) /(1 \pm f(z))^{2}=(\omega / \xi)\left((1+\xi)^{2} /(1 \pm \omega)^{2}\right)\left(z /(1 \pm z)^{2}\right)$. (Received July 6, 1956.)

\section{W. B. Stenberg: On sequences with divergent total variation. II.}

These results are an extension of the author's paper (Proc. Kon. Nederl. Akad. v. Wetensch. Ser. A vol. 58 (1955) pp. 178-190.) A sequence $a_{1}, a_{2}, \ldots$ is called "admissible" if it possesses the properties: (I) $a_{n}>0$ for $n=1,2, \cdots$, (II) $\lim _{n} a_{n}=0$, (III) $\sum_{n} a_{n}=\infty$. A sequence is called "singular" if it possesses together with properties (I), (II), (III), the property: (IV) for each subsequence $c_{1}, c_{2}, \cdots$, one has $\sum_{n} c_{n}=\infty$ if and only if $\sum_{n}\left|c_{n}-c_{n+1}\right|=\infty$. A necessary and sufficient condition that one can reorder all of the terms of an admissible sequence $a_{1}, a_{2}, \ldots$ to form a singular sequence is that $N(1 / x)=o(x)$ as $x \rightarrow \infty$. Here $N(1 / x)$ denotes the number of terms of $a_{1}, a_{2}, \cdots$ which are $>1 / x$. It is further shown that given any admissible sequence $a_{1}, a_{2}, \ldots$ one may construct a reordered subsequence $b_{1}, b_{2}, \ldots$ having the property: if $c_{1}, c_{2}, \ldots$ is a subsequence of $b_{1}, b_{2}, \ldots$ and $\alpha_{1}, \alpha_{2}, \cdots, \alpha_{p}$ are numbers, then for all $n, \sum_{k=1}^{n}\left|\alpha_{1} c_{k}+\cdots+\alpha_{p} c_{k+p-1}\right| \geqq\left(\left|\alpha_{1}\right|+\cdots+\left|\alpha_{p}\right|\right) \cdot \sum_{k=1}^{n+p-1} c_{k}-K b^{*}$. Here $K$ depends only on $p$ and the numbers $\alpha_{1}, \alpha_{2}, \cdots, \alpha_{p}$, and $b^{*}$ denotes the largest term in the sequence $b_{1}, b_{2}, \cdots$. (Received July 9,1956 .)

\section{C. A. Swanson: Differential operators with perturbed domains.}

Consider a second order ordinary linear differential operator $L$ on a half-open real interval $(0, b](b>0)$ which contains no singular points. The basic operator $A_{0}$ is defined on this interval as a restriction of $L$ to a suitable domain $D_{0}$ of the Hilbert space $\mathscr{L}^{2}(0, b)$. Let $[a, b]$ be a closed subinterval of $(0, b]$, where $a$ is a small positive number. A perturbed operator $A_{a}$ on $[a, b]$ is a restriction of $L$ to a perturbed domain $D_{a}$. It is proved under certain assumptions on the basic operator that for each characteristic value $\Lambda$ of $A_{0}$ there exists a characteristic value $\lambda(a)$ of $A_{a}$ which is developable in an asymptotic expansion with leading term $\Lambda$, valid as $a \rightarrow 0$. Furthermore, the characteristic function $y(x, \lambda)$ of $A_{a}$ possesses an asymptotic expansion valid as $a \rightarrow 0$ uniformly in the interval $[a, b]$. These expansions are not asymptotic power series, but are asymptotic expansions of a more general type. (Received July $5,1956$. )

722. R. C. Thorne: Asymptotic solutions of differential equations with a turning point and singularities.

Let $u$ be a large positive parameter and $f(\zeta)$ a regular function of $\zeta$ in a domain D. Olver (Phil. Trans. Roy. Soc. London, Ser. A. vol. 249, p. 95) has shown that if $f(\zeta)=0\left(|\zeta|^{-1 / 2-\sigma}\right)(\sigma>0)$ as $|\zeta| \rightarrow \infty$ in $D$, asymptotic expansions of solutions of $d^{2} w / d \zeta^{2}=\left\{u^{2} \zeta+f(\zeta)\right\} w$ exist which are valid uniformly with respect to $\zeta$ in unbounded sectors of $D$ and over $\zeta=0$. By a preliminary $z-\zeta$ transformation and application of this theory one can derive asymptotic expansions for solutions of $\left(^{*}\right) d^{2} w / d z^{2}=$ 
$=\left\{u^{2} z^{-2}\left(z_{0}-z\right) p(z)+z^{-2} q(z)\right\}$, valid over the turning point $z=z_{0}$ but not valid over $z=0$ since $z=0$ corresponds to a point $|\zeta|=\infty$ for which $f(\zeta)=O(|\zeta|) ; p(z)$ and $q(z)$ are regular functions of $z$ in $D$. The author shows that if $\left(^{*}\right)$ is modified, the asymptotic solutions are valid over $z=0$. When $\left(^{*}\right)$ has a regular singularity at infinity or an irregular singularity of rank one at infinity, $\left(^{*}\right)$ can be modified so that the asymptotic solutions of $\left({ }^{*}\right)$ are valid over all the singularities. The theory is relevant to the Legendre and Bessel equations. (Received June 18, 1956.)

\section{3t. W. R. Utz: A note on second-order nonlinear differential equations.}

The equations considered are $x^{\prime \prime}+\alpha(x) x^{\prime}+\beta(x) x=0$, (1); $x^{\prime \prime}+g\left(x^{\prime}\right)+c x=0,(2)$; wherein $x^{\prime}=d x / d t$. It is shown that if $\alpha(x)$ and $\beta(x)$ are real functions such that for all real $x, \alpha(x) \leqq 0, \beta(x)>0$ and if $x(t)$ is a solution of (1) valid for all large $t$, then $x(t)$ oscillates or, for all large $t, x(t)$ is monotone. In case $x(t)$ is monotone increasing, $\lim _{t \rightarrow \infty} x(t)>0$ and in case $x(t)$ is monotone decreasing, $\lim _{t \rightarrow \infty} x(t)<0$. As a corollary to this theorem one can show that when $c$ is a positive constant, $g(0)=0$, and for all real $z, g(z) \leqq 0$, then if $x(t)$ is a solution of (2) valid for all large $t$, then $x(t)$ is oscillatory, $\lim _{t \rightarrow \infty} x(t)=\infty$ or $\lim _{t \rightarrow \infty} x(t)=-\infty$. (Received July 5,1956 .)

\section{4t. C. J. Wallen and Francis Regan: A generalized Hansen series.}

A series of the type $H(z)=\sum a_{n} z^{r n+t} /\left(1-z^{r n+t}\right)$, where $r$ and $t$ are fixed positive integers, is a generalized Hansen series. If $\sum a_{n}$ diverges the $H$-series and the associated power series $\sum a_{n} z^{r n}$ converge at the same points within the unit circle, but if $\sum a_{n}$ converges the $H$-series converges for every $z$ of modulus not equal to unity. Further, the convergence is uniform in every closed sub-region completely contained in one of the regions of convergence. Therefore the function represented by an $\mathrm{H}$ series within its region of convergence can be expanded as a power series having coefficients which are sums of coefficients of the $H$-series; if $t$ is a multiple of $r$, relations exist which give the coefficients of the power series as unique sums of the coefficients of the $H$-series, and conversely. Employing a radial approach to a boundary point, the unit circle is established as a natural boundary for the function represented by an $H$-series with certain restrictions on the coefficients; imposing an added restriction the same result is obtained when a complex approach is employed. A condition is determined sufficient to establish the unit circle as a natural boundary when the $H$ series has positive real coefficients and converges within the unit circle. (Received April 26, 1956.)

725t. W. R. Wasow: Asymptotic development of the solution of Dirichlet's problem at analytic corners.

Let the boundary $C$ of a bounded plane region $R$ consist of a finite number of closed analytic arcs. Let $C$ be, furthermore, composed of a finite number of closed arcs on each of which there is prescribed an analytic function of the arc length $s$ such that the resulting function $f(s)$ is continuous on $C$. The solution $u(x, y)$ of the Dirichlet problem $\Delta u=0$ in $R, u=f(s)$ on $C$, has, in general, singularities at the corners, i.e. at the points where $C$ or $f(s)$ is not analytic. By means of methods introduced by H. Lewy and extended by R. S. Lehman it is shown that $u$ is the real part of an analytic function of $z=x+i y$ which possesses at every corner $z=a$ an infinite asymptotic series development. Let $\pi \alpha, 0<a \leqq 2$, be the interior angle at the corner, then this expansion is a power series in $(z-a)$ and $(z-a)^{1 / \alpha}$, if $\alpha$ is irrational, and in $(z-a)$, 
$(z-a)^{1 / \alpha},(z-a) \log (z-a)$, if $\alpha$ is rational. The partial derivatives of $u$ have at $a$ the same order of magnitude as those of $u_{1}(x, y)+\operatorname{Re}\left[(z-a)^{1 / \alpha}\right]$ or as those of $u_{1}(x, y)$ $+\operatorname{Re}\left[(z-a)^{1 / \alpha} \log (z-a)\right]$, according as $\alpha \neq 1 / m$ or $\alpha=1 / m$ ( $m$ an integer). Here, $u_{1}(x, y)$ is a function all of whose derivatives are bounded at $z=a$. (Received May 2, 1956.)

\section{6t. Y. K. Wong: A generalization of Toeplitz's theorem in Hilbert} space.

Toeplitz proved that if $A$ is bounded, then a bounded left inverse $L$ exists if and only if $\sum_{1}^{n} \bar{x}_{i} x_{i} \leqq c \sum_{1}^{n} \bar{x}_{i} g_{i j} x_{j}$ where $g_{i j}=\sum_{1}^{\infty} \bar{a}_{t i} a_{t j}$ for all $n$, and a similar equivalent condition holds for the existence of a bounded right inverse $R$, and $L=R$. As $A$ is bounded, $u^{*} A=0$ implies $u=0$ and $A v=0$ implies $v=0$. In this paper we consider the elements in a locally compact, connected topological field. We generalize Toeplitz's theorem to unbounded infinite matrices $A$ where the columns of $A$ and of its conjugate-transpose $A^{*}$ are Hilbert vectors. The graphs of the linear transformations induced by such matrices are not closed; consequently a new method has to be developed for our purpose. Although bounded left and right inverses $L, R$ exist, they are unequal. However, we can construct the inverse $A^{-1}$ by $A^{-1}=R+(I-F) L+D$ $=L+R(I-E)+D$, where $A D=D A=0$ and $E, F$ are projections such that $E A=A F$ $=A$. The inverse $A^{-1}$ is not unique, as $u^{*} A=A v=0$ for some Hilbert vectors $u \neq 0$, $v \neq 0$. (Received July 5,1956 .)

\section{F. M. Wright: On C-fractions belonging to a set $\Gamma$.}

In this paper, the author considers the set $\Gamma$ consisting of all $C$-fractions $C(w)$ having the property that the corresponding power series $Q(w) \equiv \sum_{n=0}^{\infty} \mu_{n} w^{n+1}$ of $C(w)$ is such that $P(z) \equiv Q(1 / z)$ has a $J$-fraction expansion. It is first noted that a method discussed by the author for essentially absolutely regular elements of $\Gamma$ can also be used to give a fairly simple and direct approach to formulas corresponding to results of W. T. Scott for the entire set $\Gamma$. It is then shown that a technique employed by the author for absolutely regular elements of $\Gamma$ in order to obtain from the above formulas certain determinant formulas for the coefficients of $C(w)$ as well as results on the usual persymmetric determinants $\Delta_{1, n}$ can also be used to obtain similar information for more general elements of $\Gamma$. It is to be noted that this information corresponds to results obtained for absolutely regular $C$-fractions by W. T. Scott and H. S. Wall (Annals of Mathematics, 1940) as well as E. Frank (American Journal of Mathematics, 1946). An additional result is obtained by the techniques mentioned relative to conditions under which the determinants $\Delta_{1, n}$ may equal 0 for two successive integers n. (Received July $3,1956$. )

\section{Applied Mathematics}

728. C. M. Ablow: Wave refraction at an interface. Preliminary report.

A plane wave in one of two perfect gases moves toward the parallel plane interface between the gases. The wave is either continuous or headed by a shock front weak enough that entropy changes may be neglected. Using Riemann's solution of the appropriate hyperbolic partial differential equation, four equations are derived giving the details of the reflected and refracted wave motions. The equations are of first order integro-differential or implicit functional form depending on the boundary 
conditions and must be solved simultaneously for four functions of a single independent variable. The equations are suitable for numerical step-by-step solution. (Received June 25, 1956.)

729. W. Band, J. L. Brenner and P. A. Clement (p): The eigenvectors of a matrix and the second quantization.

A certain matrix of Jacobi $\left(a_{i j}=0,|i-j|>1\right)$ arises in calculating rate processes by second quantization. It is proved that its eigenvalues are the same as those of a matrix due to Sylvester (Muir and Metzler, Theory of determinants, 1933, p. 546), and some properties of the eigenvectors are established. Each eigenvector corresponds to a certain mixed complexion of a physical system. The entropy of each pure complexion would be zero. Certain weighted sums of the components of the eigenvectors give the expected value of the energy and of the dispersion of the populations. By computing the latter quantities in closed form, it is determined what strategy the system should employ to enjoy maximum stability subject to constancy of the rate process in question. (Received May 28, 1956.)

\section{E. H. Bareiss: Numerical solution of the multigroup Boltzmann} transport equation for nuclear reactor design.

A code for the numerical solution of the one-dimensional multigroup Boltzmann Transport Equation is described. Large scale computations are being performed on the NORC (Naval Ordnance Research Computer) for the Westinghouse Atomic Power Division. The method for the numerical solution of the system of integrodifferential equations, arising in this problem will be discussed. Some actual but unclassified numerical results obtained by this code will be presented. (Received July 6 , 1956.)

\section{1t. H. W. Becker: Pythagorean off-resonance systems.}

Useful in teaching electronics fundamentals, the Diophantine system (1) $R^{2}+X_{L}^{2}$ $=Z_{L}^{2}, R^{2}+X_{C}^{2}=Z_{C}^{2}, R^{2}+X^{2}=Z^{2}, X=\left|X_{L}-X_{C}\right| \neq 0$ requires (2) $R=2 a b c d e f, X_{L}$, $Z_{L}=c \operatorname{def}\left(a^{2} \mp b^{2}\right) ; X_{C}, Z_{C}=a b e f\left(c^{2} \mp d^{2}\right) ; X, Z=a b c d\left(e^{2} \mp f^{2}\right) ; X=(a c+b d)(b c-a d)$, $e f /\left(e^{2}-f^{2}\right)=a b c d /(a c+b d)(b c-a d)$. This has the parametric solution (3) $a, c$ $=g\left(g^{2} \mp h^{2}\right) ; b, d=h\left(g^{2} \pm h^{2}\right) ; e, f=g \pm h$. Each such solution yields a transformed solution (4) $a, b \rightarrow(a \pm b)(c \pm d) ; c, d \rightarrow e(a-b)(c+d), f(a+b)(c-d) ; e, f \rightarrow e, f$. Thus $R, X_{L}, Z_{L}, X_{C}, Z_{C}, X, Z=60,11,61,91,109,80,100 \rightarrow 3003,26596,26765,30600$, $30747,4004,5005$. But numerical solutions are best obtained empirically: of the 25 known with $R \ngtr 3003$, only 6 come under (3) or (4), an algebraic virility or coverage of only $24 \%$. The problem is equivalent to Alliston's problem: to find a Pythagorean and a Hero triangle with a side and the area in common, Math. Snack Bar (Cambridge, 1936) p. 122. On the previous page, he proposed the problem of a $P \Delta$ and $2 \mathrm{H}$ 今 with side and area in common; his solution is in numbers of 5 and 6 digits. But the simplest solution is the set of $\$$ with sides $120,209,241 ; 123,209,218 ; 169,209$, 150. A twin solution of (1) satisfying (5) $X=X_{L} \pm X_{C}$ with either sign is $R=120$, $X_{L}=209, X_{C}=182$. (Received April 24, 1956.)

\section{J. S. Bendat: Zero crossings of a sine wave plus random noise.}

A new simple formula is derived for the expected number of zeros per second of a sine wave plus random noise. The main difference between this work and a previous study by Rice (BSTJ, vol. 27, Jan. 1948) is that whereas Rice considers a single sine 
wave of fixed amplitude, phase and frequency, the present formulation allows the sine wave to be a representative member of a "partially ergodic" stationary random process, an ensemble of records varying in amplitude and phase, fixed in frequency only. Permitting a random variation in amplitude and phase agrees more closely than before with many physical problems. The final result is expected on the average even if the process is not "partially ergodic." The formula obtained in this paper agrees with the known Rice result for random noise alone; it also coincides with the required identity for a sine wave alone. In combined situations, the formulas disagree. Procedures for measuring the expected number of zeros per second in a finite time period are discussed, as well as the question of how this information, together with other statistical properties, may be used to determine the fixed frequency of the sine wave. (Received June 27, 1956.)

\section{R. L. Brock: Mathematics as applied to the analysis of electronic detection systems.}

The analysis of electronic detection and control systems is discussed from the standpoint of the applied mathematician. Time dependent input functions for such systems consist of the sum of an unwanted random noise term and a term which represents the information desired for detection or control. Because of the random character of the input functions the analysis of such systems is necessarily statistical in nature. An effort is made to trace briefly the mathematical development in this relatively new field of application. Particular attention is given to the way in which methods of mathematical statistics enter into these studies along with such analytic topics as differential equations, special functions, integral equations and integral transforms. Three approaches to the analysis and optimization of systems subject to random input functions are considered. These are each in turn based on statistical decision theory, theory of probability distributions and autocorrelation analysis. The present state of development is indicated for each and important areas for extended mathematical effort are noted. Typical are the areas of nonlinear systems, nonstationary random processes and non-Gaussian processes. (Received July 5, 1956.)

734t. F. H. Brownell: A simplified model for a mathematical study of the perturbation theory of the Lamb shift.

It has long been questioned whether the divergences arising in quantum electrodynamics using formal perturbation expansions are due to the invalidity of these perturbation expansions or to other mathematical difficulties glossed over in the present quite formal formulation used by the physicists. In order to study this question, we consider a radically simplified model of quantum electrodynamics in which spins and negative energy states of electrons and polarization of photons are omitted and in which only a finite number of photon oscillators, depending on $b$ and $R$, are allowed. $b$ here is the linear spacing between photon oscillators and $R$ the cut-off radius in phase space. Under this model we study the shift in energy levels of the bound electron states of the hydrogen atom due to interaction with the radiation field as computed by formal perturbation series, and take the limit as $b \rightarrow 0^{+}$and $R \rightarrow+\infty$. For fixed $b$ and $R$, it is clear that the theorems of Rellich dealing with the perturbation of isolated point spectra of finite multiplicity for self-adjoint operators justify these perturbation expansions. It is also easy to prove that although the lowest nontrivial order energy term diverges as $b \rightarrow 0$ and $R \rightarrow+\infty$ (which the physicists remove by dubious renormalization) the difference in this term for two different unperturbed states converges, 
agreeing with the experimental measurement of the Lamb shift. It remains to determine what happens to the sum of the series, which equals the total energy, as $b \rightarrow 0^{+}$ and $R \rightarrow+\infty$. Also we investigate the modifications arising upon adding the complications of electron spins and photon polarization, and positive-negative energy states of electrons. (Received July 9, 1956.)

\section{J. W. Carr: Languages for digital computers.}

Languages for the description of the solution of problems using general purpose digital computers have thus far been informal and generally unsatisfactory. For "automatic programming," descriptions are needed which allow manipulation of algorithms by other algorithms, and can be easily used by both machine and human.

Simple methods of abstraction for retention of commonly used algorithms (subroutines) are required. The language must be "regular," in the sense of preventing incorrect numerical operations on syntax and incorrect syntactical operations on numbers. A survey of the methods possible from the flow diagram through the PittsMcCulloch-Kleene-von Neumann "nerve net theory" to the language of recursive function theory indicates certain possible directions of usage. (Received July 25, 1956.)

\section{P. L. Chambré: Chemical surface reactions in flow systems.}

A general basis for the analysis of catalytic surface reactions of arbitrary complexity in both external and internal hydrodynamic flows is presented. The mathematical formulation leads to a system of singular integral equations of the Volterra type for the thermodynamic state parameters of the reactants. The kernels depend on the specific hydrodynamic character of the flow. Applications to boundary layer and fully-established flows of laminar as well as turbulent character have been made. (Received July 5, 1956.)

\section{7t. Jim Douglas, Jr.: A note on the numerical solution of parabolic} differential equations.

The object of this paper is to discuss the relation between two techniques of demonstrating convergence of solutions of difference equations to those of the parabolic differential equation $a(x) u_{x x}+b(x) u_{x}=u_{t}$. The two methods are based on, respectively, a maximum principle and stability analysis. It is shown that, for reasonable analogues of the differential equation, a difference satisfying the maximum principle must be stable. An example of a stable difference equation which does not satisfy the maximum principle is constructed; hence, the stability analysis technique is properly more general than the maximum principle method. (Received July 5,1956 .)

738. Jim Douglas, Jr.: The application of stability analysis in the numerical solution of quasi-linear parabolic differential equations.

It has been shown previously (Douglas, Pacific Journal of Mathematics, 1956, to appear) that, for reasonable initial and boundary conditions, the solution of the first boundary value problem for the quasi-linear parabolic differential equation $u_{x x}$ $=f(x, t, u) u_{t}$ can be approximated by the solution of the backward difference equation $\Delta_{x}^{2} w_{i, n+1}=f\left(x_{i}, t_{n}, w_{i n}\right)\left(w_{i, n+1}-w_{i n}\right) / \Delta t$, where $\Delta_{x}^{2}$ is the centered, second $x$-difference. As the error is $O\left((\Delta x)^{2}+\Delta t\right)$, more computation is required than is desirable. The object of this paper is to prove that the solution of the Crank-Nicolson type difference 
equation $\Delta_{x}^{2}\left(w_{i, n+1}+w_{i n}\right)=2 f\left(x_{i}, t_{n+1 / 2}, w_{i n}+\Delta t \Delta_{x}^{2} w_{i n} / 2 f\left(x_{i}, t_{n}, w_{i n}\right)\right)\left(w_{i, n+1}-w_{i n}\right) / \Delta t$ converges $L_{2}$-wise to $u$ with an error which is $O\left((\Delta x)^{2}+(\Delta t)^{2}\right)$. The proof is based on an extension of a stability analysis argument (Douglas, Journal of Society for Industrial and Applied Mathematics, 1956, to appear). Generalizations to other difference equations and higher dimensions are given. (Received July 6, 1956.)

739. T. C. Doyle: $A$ method of matrix inversion applicable to a digital computer.

Associated with any real nonsingular $n \times n$ matrix $A$ is the related symmetric matrix $a=A^{T} A$, to be regarded as the coefficient matrix of a positive-definite quadratic form $y^{T} a y$. Since $A^{-1}=a^{-1} A^{T}$, the inversion of $A$ is reduced to finding $a^{-1}$. To form $a^{-1}$, the linear system $a x=b$ is transformed into $H^{T} a H \xi=H^{T} b$ by $x=H \xi$, where $H$ is a triangular matrix determined so that $H^{T} a H=d^{-1}$ is a diagonal matrix. Then $\xi=d H^{T} b$ and $x=H d H^{T} b$ so that $a^{-1}=H d H^{T}$. $H$ is readily machine constructed and the elements of $a^{-1}$ appear as summations over the ascending ranges $(1)(1,2)$ $(1,2,3), \cdots,(1,2, \cdots, n)$. A machine storage plan and addressing scheme is illustrated for $n=3$. (Received March 28, 1956.)

740. M. L. Friberg and C. R. Cassity (p): A rational description of explosive-induced shock waves in metals. Preliminary report.

Although the equations describing the motion of elastic waves are well-known, the conditions under which these equations may be applied are not well-established and very few calculations which might be used to compare with the extensive test data available have been made heretofore. Some new solutions of the wave equation are compared with the results of a series of tests. (Received July 2,1956.)

\section{Bernard Friedman: Two theorems on wave propagation.}

(1) It is shown that the equation $u^{\prime \prime}+(\lambda-q(x)) u=0$ over the interval $(0, \infty)$ with the boundary condition $u(0)=0$ must have virtual eigenvalues if $\int_{0}^{\infty} x|q(x)| d x<\infty$ and $q(x) \neq 0$. (2) A result of Rellich (Jbr. Deutschen Math. Verein vol. 53 (1943) pp. $57-64)$ is generalized to prove the uniqueness of the solution of $\Delta u+(\lambda-q) u=0$ with Dirichlet boundary conditions if $\iint|q| r^{-2} d r d \Omega<\infty$. (Received July 9, 1956.)

742. W. T. Guy, Jr.: Integral transform solution of a nonlinear differential equation.

An approximate solution to a Duffing differential equation was obtained using integral transforms and the convergence of the process was presented. (Received July 9, 1956.)

743. Isidore Heller: On linear systems with integral valued solutions, I.

The constraint matrix of the Hitchcock-Koopmans transportation model has the property: whenever a column is a linear combination of linearly independent columns, the coefficients are integers (and therefore necessarily \pm 1 or 0 ). This property permits a simplification in the computational procedure of the "simplex method" as was shown by G. Dantzig (Activity analysis of production and allocation), and assures that all basic solutions are integral valued whenever one integral valued solution 
exists. C. Tompkins and the author (Annals of Mathematics Studies, no. 38) have shown the mentioned property to hold for an extended class of matrices. J. Kruskal and A. Hoffman (ibid.) have related this property, which they termed "unimodular property," to partly ordered sets. The present note, unifying and extending the cited results, proves the following statements (see below). (Received May 31, 1956.)

\section{Isidore Heller: On linear systems with integral valued solutions,} II.

1. If $b_{1}, b_{2}, \cdots, b_{k}$ are linearly independent vectors in a linear vector space, then the set $D=\left\{ \pm b_{i}, b_{i}-b_{j}\right\}$ is unimodular. 2. $D$ is maximal for its dimension, in the sense that the span of $D$ does not contain a unimodular set properly containing $D$. 3. Theorem: If a unimodular set of dimension $k$ contains at least $k(k+1)$ vectors, not counting the nullvector, then it is of type $D$ (and hence contains exactly $k(k+1)$ vectors). 4 . For $k \geqq 4$ there are $k$-dimensional maximal unimodular sets which are not of type $D$ (they necessarily have less than $k(k+1)$ elements). The unimodular sets of type $D$ containing the unit vectors, that is the representations of $D$ in coordinates relative to the different bases in $D$, are easily described by associating a tree of $k+1$ vertices and $k$ oriented and numbered segments to each basis in $D$; this yields 1-1 correspondence between representations and trees. (Received May 31, 1956.)

745. William Karush: On a minimization problem involving convex functions.

Let $f\left(x_{1}, x_{2}, \cdots, x_{n}\right)=\sum_{i=1}^{n} f_{i}\left(x_{i}\right)$, where each $f_{i}$ is a real-valued convex continuous function over the real numbers. In various applications, e.g., in mathematical economics, one encounters the problem of minimizing $f$ with the $x_{i}$ subject to linear restraints. Let $A, B$ with $A \leqq B$ be arbitrary and restrain $x_{i}$ by the conditions $A \leqq x_{1}$ $\leqq x_{2} \leqq \cdots \leqq x_{n} \leqq B$. In this paper, the function $F(A, B)=\min f$ over such $x_{i}$ is studied. It is shown that $F$ satisfies the functional equation $F(A, C)=F(A, B)$ $+F(B, C)-F(B, B), A \leqq B \leqq C$. From this is obtained the decomposition $F(A, B)$ $=M(A)+N(B)$, where $M, N$ are explicitly determined monotonic convex functions. These properties of $F$ also hold in the continuous case where $F(A, B)=\min \int_{0}^{1} f(t$, $x(t)) d t$ over all monotonic functions $x(t), 0 \leqq t \leqq 1$, satisfying $A \leqq x(0) \leqq x(1) \leqq B$; here $f(t, x)$ is convex continuous in $x$ for each $t$, and $f$ is sufficiently smooth as a function of $t$ and $x$. (Received July 9,1956.)

\section{6t. Erwin Kreyszig: Stable oscillating systems having quadratic} damping.

The equation (1) $\ddot{q}-(\beta / 2)(\operatorname{sgn} \dot{q})\left(1-\alpha^{2} q^{2}\right) \ddot{q}^{2}+\left(\gamma^{2} / 2\right) f(q)=0, \beta>0$, where $q(t)$ is the elongation and $t$ the time, may be considered as a generalization of the well known van der Pol equation. (1) can be transformed into (2) $V^{\prime}-\beta(\operatorname{sgn} \dot{q})\left(1-\alpha^{2} q^{2}\right) V+\gamma^{2} f(q)$ $=0$, where $V=\dot{q}^{2}$. Small oscillations increase while large oscillations decrease; in both cases the "maximum amplitude" $Q_{n}(t), n=1,2, \ldots$ (i.e. the value of $q(t)$ for which $\dot{q}(t)=0)$ tends to a limit $q=Q_{s}$ if $n \rightarrow \infty$, i.e., $t \rightarrow \infty$. From the first integral of (1) bounds for $Q_{s}$ can be obtained for every type of the restortion force which is of practical interest. For example, if $f(q) \geqq 0 \quad(q>0), f(q) \leqq 0(q<0), f(q) \not \equiv 0$, then $\left|Q_{s}\right|>3^{1 / 2} /|\alpha|$. If $f(q)=q^{2 m+1}, m=0,1, \cdots$ the following results hold: I. $\left|Q_{s}\right|<q_{0}$ where $q_{0}$ is the smallest zero of $h(z)=\exp (2 \beta q) P\left(2(m+1) / 3, c q^{3}\right)-P(2(m+1) / 3$, $\left.-c q^{8}\right), c=\alpha^{2} \beta / 3$, and $P(m, x)=\int_{0}^{x} e^{-t} t^{m-1} d t$ is the incomplete Gamma function. II. The ratio $\left|Q_{n}\right| /\left|Q_{n+1}\right|$ depends on $u=\alpha / \beta$ only and is a monotone increasing function of $u$. Similar results can be obtained in the case where $f(q)$ is an arbitrary polynomial of $q$. 
Approximate solutions of (1) can be derived by means of a refinement of the method of slowly varying amplitude and phase. (Received June 28, 1956.)

747. T. C. H. Li: Tip effect of an oscillating finite thin wing in supersonic flow.

The tip effect of a swept, oscillating, finite thin wing, with supersonic leading edges in supersonic flow, is determined by mapping the region on the imaginary diaphragm off the wing onto the disturbing region on the wing. This is accomplished by a linear transformation, independent of the coordinates of the receiving point. A set of aerodynamic influence coefficients for the disturbing region is derived which may be handled conveniently by any type of high-speed computer. Results obtained for the rectangular wing are in very good agreement with those of Acum and those of Watkins. (Received July 5,1956 .)

\section{L. E. Malvern: Eigenfunction series for beam-column with elastic end support.}

The use of series of eigenfunctions of a column to represent deflection under given lateral loads and sub-critical axial compressive load is well-known for the cases in which each end is simply-supported, fixed, or free. The usual orthogonality property of the second derivatives of the eigenfunctions, which facilitates determination of the series coefficients by use of the Principle of Virtual Displacements, is lacking when the ends are elastically supported against deflection and/or rotation. By properly including the virtual work of the elastic support forces, however, it is possible in this case also to obtain the coefficients without solving simultaneous equations. The formula obtained for the coefficient $a_{m}$ in the series $\sum a_{m} v_{m}(x)$ then has added in the denominator the terms $A_{0} v_{m}^{2}(0)+A_{L} v_{m}^{2}(L)+B_{0}\left[v_{m}^{\prime}(0)\right]^{2}+B_{L}\left[v_{m}^{\prime}(L)\right]^{2}$, but does not otherwise differ from the usual result. Here $A_{0}$ and $A_{L}$ are the spring constants of the supports against deflection at the ends $x=0$ and $x=L$ of the beam-column, while $B_{0}$ and $B_{L}$ are the spring constants of the restraints against rotation. (Received July 9,1956.)

749. W. D. Maness: $A$ generalized Green's relation $R$ for $a(n-1)$ dimensional Cartan hypersurface.

An analytic variety which sustains asymptotic parametric curves is known as a Cartan variety. A generalization of Green's relation $R$ is given for a line $l$ and a linear $(n-2)$-space $L$ in the tangent hyperplane to a $(n-1)$-dimensional Cartan hypersurface. The power series expansion in local nonhomogeneous coordinates for the equation of the hypersurface is obtained. The power series is simplified by choosing a suitable reference frame. The complete geometric characterization of this reference frame is given and a generalization of the edges of Green for $n$-dimensional space is obtained by use of the generalized relation $R$. The power series obtained and the reference frame chosen are shown to specialize to that obtained by G. M. Green using Wilczynski's normal coordinates (Trans. Amer. Math. Soc. vol. 20 (1919) pp. 79-153). (Received July $5,1956$.

750. William Nachbar: Note on exponential approximations for the rectangular plate.

Deflections and stress resultants for a laterally-loaded rectangular plate with two opposite edges simply supported can be computed by Fourier series expansions. Many examples of this are given in S. Timoshenko Theory of Plates and Shells, McGraw-Hill, New York, 1940, Chapters V and VI. Slow convergence, particularly of the series 
representing the stress resultants, is sometimes a practical difficulty of this method, however. There are possible advantages to be gained from expanding portions of the Fourier coefficient of order $n$ in a power series in the factor $e^{-\alpha n}$, where $\alpha$ is proportional to the ratio of the lengths of sides, and then rearranging the main series. This note discusses the advantages in using such a technique, in particular for obtaining simple formulas for approximating the sums of the Fourier series. The plate with one free edge and three edges simply supported is used as an example. (Received July 6 , 1956.)

\section{Walter Noll: On isotropic functions of linear transformations.}

Isotropic functions are of great importance in modern continuum mechanics. They are of the form $f(x)$ or $F(x)(f(x)$ real; $x, F(x)=$ linear transformations of a real Euclidean vector space) and satisfy $f(x)=f\left(R x R^{-1}\right)$ and $R F(x) R^{-1}=F\left(R x R^{-1}\right)$ for all orthogonal (proper orthogonal) transformations $R$. They are called symmetric if $x$ and $F(x)$ are restricted to be symmetric. The properties of such functions and of their differentials (in the sense of Hamilton) are investigated. In particular, the functions $a^{(k)}(x)$ ( $=k$ th principal invariant of $x$ ), $x^{n}$, and $x^{1 / n}$ are studied. Any symmetric isotropic $f(x)$ can be expressed in terms of the $a^{(k)}(x)$, and any symm. isotrop. $F(x)$ has a representation $F(x)=\sum_{k=0}^{n-1} \phi_{k}(x) x^{k}$, where the $\phi_{k}(x)$ are real valued and symmetric isotropic. The continuity and differentiability properties of these representations are discussed. (Received July 6, 1953.)

\section{2t. F. Reza: A qualitative theorem on the response of linear systems in the time domain.}

Let $f(t)$ be a continuous single-valued bounded function of a real variable $t$ (time) whose derivative has a finite number of real roots. $f(t) \equiv 0$ for $t<0$. A shape function $C_{n}$ is associated with $f(t)$ based on the following: (a) Denote by $t_{1}<t_{2}<t_{3} \cdots<t_{n}$ the real values of $t_{j}$ for which $d f\left(t_{j}\right) / d t=0$. (b) Let $C_{n}$ be a finite sequence of $j=1,2, \cdots, n$ so that the corresponding $f\left(t_{j}\right)$ 's will be in order of their values, the smallest being at the left of this finite sequence. $C_{n}$ gives some qualitative measure of the shape of $f(t)$ on the real axis. Two functions having same shape functions are said to have the same shape. Theorem: Let $C_{n}$ be the shape function of a function $f(t)$ (as described in the above). (i) There exist finite linear passive lumped bilateral electrical networks $N$ at rest at the time $t=0$, such that for an applied unit impulse stimulus, applied at $t=0$ the response of the system has $C_{n}$ for its shape function. (ii) The networks $N$ cannot contain less than $n+1$ reactive elements if $N$ is confined to resistor-capacitor or resistor-inductor networks. (iii) The lower limit is possible, i.e., it is possible to synthesize a network of the type described in (i) with only $n+1$ reactive elements. (Received July $9,1956$.

\section{3t. L. S. Shapley: Game solutions with arbitrary components.}

A class of solutions to the $n$-person simple game, $n \geqq 4$, with minimal winning coalitions $\{2,3,4, \cdots, n\},\{1,3,4, \cdots, n\},\{1,2,4, \cdots, n\}$ is exhibited in which one component, $J$ is an arbitrary closed subset of the $(n-3)$-dimensional set of imputations of the form $\left(0, a, a, \alpha_{4}, \cdots, \alpha_{n}\right), a>0$ (in the $0-1$ normalization). The remaining component depends upon $J$, and is closed, connected, and has maximum dimension $n-2$. The arbitrariness of $J$ permits the construction of "pathological" examples that dispose of several conjectures regarding the regular behavior of solutions. (Received July 6, 1956.) 


\section{H. E. Stelson: Laplace transforms applied to interest func- tions.}

Interest functions with constant rate may properly be considered as step functions, so that difference equations may be determined and resolved by means of Laplace transforms. The analog is continuous interest where differential equations are used in evaluation. Generalized interest functions may be derived by use of Laplace transforms. Interest functions with constant time may use Laplace transforms to determine an unknown rate. (Received July 6, 1956.)

755t. R. H. Wasserman: A formulation and solutions of the equations of ideal fluid flow.

Following the work of N. Coburn (Michigan Mathematical Journal, vol. 1, no. 2, (1952)) the laws of steady ideal fluid flow are formulated intrinsically in terms of the unit trihedron of the stream lines for the two cases of incompressible fluids and polytropic gases subject only to the condition that the entropy be constant along a stream line. When the stream lines are taken as coordinate curves the equations governing the flow are obtained as a set consisting of two linear differential equations and one algebraic equation for a scalar function of the pressure. With these equations, given geometrical properties of the congruence of curves formed by the stream lines, additional general properties of the flows are obtained. In particular, the question of existence of fluid flows having stream surfaces on which the stream lines are geodesics or asymptotic curves can be answered in some cases. The answer is affirmative when the stream surfaces are concentric circular cylinders and the stream lines are geodesics on these cylinders. Moreover, the entire class of these helical flows is obtained explicitly in terms of several arbitrary functions, reflecting a considerable variety of geometrically and thermodynamically distinct flows. (Received July 6, 1956.)

\section{H. F. Weinberger: Forced vibrations and nonorthogonal pro-} jection.

Let $L$ be a differential operator with boundary conditions such that the bilinear form $\int u L u$ is positive definite. The forced vibration of a system on which the force corresponding to displacement $u$ is $L u$ is governed by the equation $L u-k^{2} \rho u=f$. For $k$ sufficiently large the natural norm $\int u\left(L u-k^{2} \rho u\right)$ is not definite. Hence the Schwarz inequality method of Diaz [Collecteanea Math. 4 (1951) fasc. 4] and the equivalent hypercircle method of Prager and Synge [Quarterly of Applied Mathematics vol. 5 (1947) pp. 241-269] do not work. Just as in these methods, we consider functions $v$ satisfying the same differential equation and natural boundary conditions as $u$ and functions $w$ satisfying only the remaining boundary conditions for $u$. We use the norm $\|\phi\|^{2}$ $=\int u\left(L u+c^{2} \rho u\right)$ where $c^{2}$ is any positive constant. In this norm $(v-u)$ and $(w-u)$ are not orthogonal. It can be shown by direct computation that $\|w-u\| \leqq K\|w-v\|$ for all $v$ and $w$, where $K$ depends on the distance of $k^{2}$ from the nearest eigenvalue of $L u-\lambda \rho u=0$ and is singular when $k^{2}$ is an eigenvalue. Since $w-u$ satisfies only boundary conditions, this inequality is insufficient for a pointwise bound for $|w-u|$ if $L$ is of second order. A geometric consideration in Hilbert space shows that the above inequality is equivalent to $\|v-u\| \leqq K\|w-v\|$. Since $w$ and $v$ are arbitrarily chosen and since $v-u$ satisfies a differential equation, this inequality gives a pointwise bound for $|v-u|$ which can be made arbitrarily small. (Received July 9, 1956.) 
757. Y. K. Wong: Note on Hurwitz's condition on dynamic stability.

The characteristic polynomial $\sum c_{n-i} \lambda^{i}$ of a real-valued square matrix $A$ of order $n$ can be determined in terms of traces $t_{p}$ of the pth powers of $A$ as follows: $c_{k}=$ $=\sum\left(-t_{1}\right)^{\alpha}\left(-t_{2} / 2\right)^{\beta} \cdots\left(-t_{n} / n\right)^{\nu} / \alpha ! \beta ! \cdots \nu$ !, where the summation extends to all $\alpha, \beta, \cdots, \nu$ such that $\alpha+2 \beta+\cdots+k \nu=k$. We can prove that $\partial c_{k} / \partial t_{p}=-c_{k-p} / p$. Hurwitz's condition for dynamical stability is expressed in terms of a sequence of determinants $d_{m}$ with its $k$ th row of the form $\left(c_{2 k-1}, \cdots, c_{k}, \cdots, c_{2}, c_{1}, 1,0, \cdots, 0\right)$, where $k=1,2, \cdots, m$ and $c_{q}=0$ if $q>n$. We show that $d_{m}(m=1,2, \cdots, n)$ is independent of $t_{2 p}$, the trace of the even power of $A$, provided that $2 m-1 \leqq n$. (Received July $5,1956$.

\section{Geometry}

\section{8t. L. M. Blumenthal: Global subsets of the $n$-sphere.}

A subset of the $n$-sphere $S_{n}$ is global provided it is not contained in any hemisphere. The paper presents new necessary and sufficient conditions for globality of certain subsets of $S_{n}$. A mapping $\phi$ of $S_{n}$ (minus its north and south poles) into itself is studied, for which the image of a point $p$ of $S_{n}$ is the point whose $i$ th coordinate is $\left(\cos p p_{i}\right) / N,(i=0,1, \cdots, n)$, where $p_{0}, p_{1}, \cdots, p_{n}$ are fixed points of the equatorial $S_{n-1}$ and $N=\sum_{i=0}^{n} \cos ^{2} p p_{i}$. It is shown that $\phi$ is an isometry on $S_{n-1}$ if and only if the basic $(n+1)$-tuple is either equilateral (and hence global on $S_{n-1}$ ) or becomes so upon reflecting one or more of its points in the origin (the center of $S_{n}$ ). (Received April 16, 1956.)

\section{W. R. Cowell: Ternary rings in relation to incidence matrices.}

Let the finite projective plane $\pi$ be coordinatized by a ternary $T$ and let $\pi^{\prime}$ be the corresponding affine plane. Then the rows and columns of the line-point incidence matrix $M$ of $\pi$ can be arranged so that permutation matrices corresponding to the latin square representation of $\pi^{\prime}$ appear as submatrices of $M$. It is then possible to interpret the ternary operation in terms of these permutation matrices. Various properties of $T$ such as linearity, associativity of addition and multiplication, and the distributive laws correspond to matrix identities. The properties of linearity and associative addition are preserved under certain transformations of the coordinate system and linearity is shown to be equivalent to a particular isotopy relation among the latin squares representing $\pi^{\prime}$. (Received July $5,1956$. )

760. K. D. Fryer (p) and I. Halperin: The von Neumann coordinatization theorem for complemented modular lattices.

The theorem of J. von Neumann, that coordinates can be introduced into any complemented modular lattice of order four or more is again considered. The proof of this theorem given previously by the authors is simplified and additional axioms are given under which this proof applies to complemented modular lattices of order three (the new axioms play the role of Desargues' theorem in projective geometry. (Received June 28, 1956.)

\section{1t. Donald Greenspan: On vertices of space curves.}

A new approach, which avoids differentiability assumptions and which has a potential topological generalization, is made in the study of vertices of $n$-dimensional space curves, $n \geqq 3$. Classes of arcs and curves called base arcs and base curves, re- 
spectively, are defined. A fundamental theorem, the Reduction Theorem, is proved first. This theorem enables one to always employ the more useful "point of intersection" rather than the "point of support" in any discussion of the point set intersection of an arc (or curve) and a hypersphere (which term includes the concept of hyperplane). Subsequent to further definitions, remarks, and lemmas, the following major results are proved: (a) Contraction Theorem. Let hypersphere $K$ be oriented and meet base $\operatorname{arc} B$ (or base curve $C$ ) in $(n+2)$ distinct points. Then it is possible to generate a descending sequence of $(n+2)$-tuples whose spans converge to zero. (b) If $P_{1}, P_{2}, \cdots, P_{n+2}$ are points of $B \cdot K$ in natural order, then the closed subarc $T$ of $B$ with initial point $P_{1}$ and terminal point $P_{n+2}$ contains in its interior at least one $K$ vertex. (c) Two Vertex Theorem. Every base curve $C$ which meets hypersphere $K$ in at least $(n+2)$ points has at least two vertices. (Received June 1,1956.)

\section{2t. Isidore Heller: On the edges of a simplex.}

The edges of a simplex, interpreted as vectors (in both orientations), have the following property: if one of them is a linear combination of linearly independent ones then the coefficients are \pm 1 or 0 . The note proves the converse: If a $k$-dimensional set of at least $k(k+1)$ vectors $\neq 0$ has the mentioned property, then there is a $k$-simplex having the vectors as its edges (Comp. the author, On linear systems with integral valued solutions, Abstract 744 above.) (Received June 4, 1956.)

763t. Robert Hermann: Global aspects of complete linear differential systems. Preliminary report.

$X$ is a $C^{\infty}$ manifold of dimension $n, t_{I}, \cdots, t_{n}$ are everywhere independent first order linear differential operators (vector fields). $V$ is a real vector space of $\operatorname{dim} . m$, $a_{1}, \cdots, a_{n}$ are $C^{\infty}$ maps $X \rightarrow E(V)$, the space of endomorphisms of $V$. Consider the differential system: $t_{i}(f)=a_{i}(f)$, to be solved for a $C^{\infty}$ map $f: X \rightarrow V$. Assume the system has locally $m$ linearly independent solutions. Let $S$ denote the sheaf of germs of solutions. Then, (1) If $X$ is simply connected, the system has globally $m$ linearly independent solutions, and, in the language of the cohomology theory of sheaves, $H^{i}(X, S)$ is isomorphic to $H^{i}(X, R)$ for $i>0$. (2) The system is elliptic in the sense of Schwartz. Corollary: On the homogeneous space of a Lie group, there is but one differential structure compatible with the action of the group. (Received June 28, 1956.)

\section{4t. Shoshichi Kobayashi: A generalization of bounded domains.}

Let $M$ be a complex $n$-dimensional manifold with a holomorphic $n$-form $\omega$ which does not vanish at any point of $M$. Let $F$ be the set of all holomorphic functions on $M$ which are square integrable with respect to the volume element $\omega \wedge \bar{\omega}$. Then $F$ is a separable Hilbert space. From a complete orthonormal basis for $F$, the generalized Bergman's kernel function can be constructed by the well known method. The generalized Bergman's metric (in general, positive semi-definite) can be defined if and only if (A) For every point $z$ of $M$, there exists an $f$ in $F$ such that $f$ is different from zero at $z$. The metric is positive definite if and only if (B) For every analytic tangent vector $V$ to $M$ at $z$, there exists an $f$ in $F$ such that $f(z)=0$ and $V(f)$ is different from zero. The metric depends only on the complex structure of $M$. A similar class of complex manifolds was given by Calabi-Washnitzer independently. A manifold with the metric above defined can be imbedded isometrically in a natural manner into the projective space over $F$ with the canonical metric. The group of holomorphic transformations of $M$ satisfying (A) and (B) is a Lie group with compact isotropic sub- 
groups. If the group is transitive on $M$, then the metric and the Ricci curvature coincide. (Received August 22, 1956.)

765. J. C. Morelock, N. C. Perry, and W. R. Hutcherson (p): Fibonacci sequence applied to quadratic transformations.

A method of finding perfect points in the neighborhood of a singular point by use of quadratic transformations and a cyclic homography is obtained by a Fibonacci sequence, provided only one quadratic transformation is used in the process. When two are used alternately a method is also obtained and a proof given for it. [W. R. Hutcherson and J. C. Morelock, Concerning a pattern for perfect points, Revista Matematica Y Fisica Teorica (Tucuman) Serie A vol. 10 (1954) pp. 187-194]. (Received May 23, 1956.)

766. T. G. Ostrom: Transitivities in finite projective planes and related topics.

The author shows that if $p, q, r, s$ are four points on a line $L$, then the perspectivities which leave $p$ and $q$ fixed and carry $r$ into $s$ all produce the same permutation of points on $L$. A similar result holds if $p$ is the only fixed point on $L$. This amounts to a kind of uniqueness for addition and multiplication as defined by Veblen and Young. Combining results of Baer and Andre, the following theorem follows: If the plane is both $p-L$ transitive and $q-L$ transitive, where $p$ is on $L$ and $q$ is not, then the plane can be coordinatized by a near field. Other results: If $n$ is an odd power of 2 and the plane is doubly transitive, it is Desarguesian. For any $n$, if the plane is transitive on quadrangles, each quadrangle generates a Desarguesian subplane. Every finite plane contains many Desarguesian configurations. (Received July 5, 1956.)

767. T. K. Pan: Exterior differential system and mapping of surfaces with associated vector fields in correspondence.

Exterior differential systems have been successfully applied to the study of certain type of problems in differential geometry (Elie Cartan, Les systèmes différentiels extérieurs et leurs applications géometriques, Hermann \& Cie., Paris, 1945). Analogous to the fifteen illustrative problems in the second part of the book, this paper investigates the surfaces in conformal correspondence which preserves associated vector fields, their indicatrices and their normal curvatures along corresponding curves. The general solution depends on six arbitrary functions of one variable. The characteristics are the curves and the indicatrices of the associated vector fields. The singular solution depends on four arbitrary functions of one variable. It consists of : equivalent or symmetrical surfaces associated with equivalent vector fields; similar developable surfaces with associated unit vector fields whose angular spreads along corresponding curves are inversely proportional to the arc lengths of these curves; similar surfaces with equal Gaussian curvatures at corresponding points and with associated vector fields whose curves are geodesics and whose angular spreads along corresponding curves are inversely proportional to the arc lengths of these curves. The problem of Cauchy is investigated. (Received June 6, 1956.)

768. R. R. Phelps: Convex sets and nearest points.

A well known theorem characterizes the convexity of a closed set $S$ in Euclidean $n$-space $E^{n}$ in terms of a "nearest point" map which assigns to each point of $E^{n}$ a nearest point in $S$. We consider, in a more general normed linear space $E$, the follow- 
ing sets which correspond roughly to the inverse images of such a map: If $z \in S$, denote by $S_{z}$ the set $\left\{x:\|x-z\|=\inf _{y \in S}\|x-y\|\right\}$ of all points having $z$ as a nearest point in $S$. If the dimension of $E$ is at least three, the statement " $S_{z}$ is convex whenever $S$ is convex" is equivalent to the existence of an inner product in $E$, while in twodimensional spaces it is equivalent to strict convexity. We say that $S$ is proximinal if for each $x \in E$ there is at least one $z \in S$ such that $x \in S_{z}$. If $E$ is smooth and strictly convex a proximinal sets $S$ is convex if and only if for each $z \in S, S_{z}$ is a cone with vertex $z$. Finally, in an inner product space, a closed set $T$ is convex if and only if there exist a set $S$ and a point $z$ in $S$ such that $T=S_{z}$. (Received July 17,1956.)

\section{9t. Geert Prins and Frank Harary: Some enumeration problems for} trees.

Using the methods of G. P6lya, Acta Math. vol. 68 (1937) pp. 145-254, and R. Otter, Annals of Math. vol. 49 (1949) pp. 583-599, several enumeration problems for trees are solved. The results include the derivation of generating functions for the number of rooted as well as free (or unrooted) trees of the following kinds: (a) labelled or multiply-rooted, (b) having a given vertex-degree partition, (c) oriented, (d) directed, (e) signed, (f) of given strength, ( $g$ ) of given type, (h) having a given diameter, (i) having a given vertex-weight distribution, ( $j$ ) homeomorphically irreducible, and also (k) having the identity group as their automorphism group. Results (a) and (c) above, as well as several others, have also been independently obtained in a recent unpublished manuscript by J. Riordan, The enumeration of labelled trees. (Received June 11, 1956.)

770. B. M. Stewart: Asymmetry of convex curves with respect to centroids of area.

Let the degree to which a point $P$ serves as a center of symmetry for a closed plane curve $C$ be measured by reflecting $C$ in the point $P$ to obtain the curve $C^{\prime}$ and considering the ratio $r(P)$ of the area common to $C$ and $C^{\prime}$ to the area bounded by $C$. A. S. Besicovitch has shown when $C$ is convex there exists a point $P$ for which $r(P)$ $\geqq 2 / 3$; and for a triangle the maximum value for $r(P)$ is $2 / 3$ which is attained only when $P$ is the centroid. We show by independent argument that if $C$ is convex, the centroid $G$ of the area bounded by $C$ has the property $r(G) \geqq 2 / 3$. We consider a conjecture of $\mathrm{P}$. C. Hammer that if $C$ is convex, there is a unique $P^{*}$ (not usually $G$ ) for which $r(P)$ is maximal. We show when $C$ is not convex that $r\left(P^{*}\right)$ may be arbitrarily small and that $P^{*}$ need not be unique. (Received July 6,1956 .)

771. T. T. Tanimoto: Minimal simple paths spanning a finite set.

Given $n$ points $P_{1}, P_{2}, \cdots, P_{n}$ in an arbitrary space, and the distance $d\left(P_{i}, P_{j}\right)$ $=a_{i j}>0$ for every pair of distinct points $P_{i}$ and $P_{j}$, we define the weight $W_{i}$ of the point $P_{i}$ by $W_{i}=\sum_{j} a_{i j}$ and the weight $w_{i j}$ of the segment $\left(P_{i}, P_{j}\right)$ by $w_{i j}=\left(W_{i}\right.$ $\left.+W_{j}\right) / 2-a_{i j}$. For any simple path $\Gamma$ (homeomorphic to a Jordan arc) through all the points with $P_{1}$ and $P_{2}$ as end points, we show that $W=\left(T_{1}+T_{2}\right) / 2-L$ where $W$ is the sum of the weights of the segments of $\Gamma$, and $L$ its length, and where $T_{1}=\sum_{i} W_{i}$ $-W_{1}$ and $T_{2}=\sum_{i} W_{i}-W_{2}$ are constants. $L$ is minimized by maximizing the quotient $W / L$. To maximize the quotient we define $q$-numbers as ordered pairs of positive numbers $(a, b)$ with addition defined componentwise and whose value is given by the quotient $b / a$. When given a finite set of $q$-numbers it is shown that the maximumvalued $r$-termed $q$-sum is found by a sequential maximization of partial $q$-sums start- 
ing with the maximum-valued term. The minimum simple path spanning the $n$ points with the end points unspecified is determined by a sequential maximization of the values of the partial $q$-sums consisting of terms $q_{i j}=\left(a_{i j}, w_{i j}\right)$ with the graphical restrictions. The segments appear in a not-necessarily-connected sequence. (Received July 6, 1956.)

\section{F. A. Valentine: $A$ three point convexity property.}

A set $S \subset E_{m}$ is said to possess the three point convexity property $P_{3}$ if for each triple of points $x, y, z$ in $S$ at least one of the closed segments $x y, y z, x z$ is in $S$. Theorem 1: Suppose $S$ is a closed connected set in $E_{2}$ (the Euclidean plane) having property $P_{3}$. Then $S$ is expressible as the union of $n \leqq 3$ closed convex sets having a nonempty intersection. A point of $S$ at which $S$ is not locally convex is called a $q$-point, and the cardinality of the set of all $q$ points of $S$ is denoted by $N$. Theorem 2: If $N$ is not an odd integer greater than 1, then the set $S$ in Theorem 1 can be expressed as the union of $n \leqq 2$ closed convex sets having a nonempty intersection. (Received July 1, 1956.)

773t. Donald Warncke and Fred Supnick: On the covering of $E_{n}$ by spheres.

Let $S_{n}$ denote the set of $n$-spheres with radii of length $\left(n^{1 / 2}\right) / 2$ and centers on the lattice points of a rectangular Cartesian coordinate system in $E_{n}$ (Euclidean $n$-space). Since $\left(n^{1 / 2}\right) / 2$ is half the largest diagonal of the unit $n$-cube, every point of $E_{n}$ falls on or within some of the spheres of $S_{n}$. For $n=1,2,3$ if an $n$-sphere is removed from $S_{n}$, then certain points of $E_{n}$ are not covered by the remaining spheres. However, for $n>3$ proper subsets of $S_{n}$ cover $E_{n}$ completely. (Let $[x]$ denote the greatest integer less than or equal to $x$.) The following theorem is established: Each point of $E_{n}(n \geqq 4)$ is on or within some $n$-sphere with radius of length $\left(n^{1 / 2}\right) / 2$ and center at a lattice point $\left(y_{1}, \cdots, y_{n}\right)$ for which $\sum_{i=1}^{n} y_{i} \equiv 0 \bmod ([n / 4]+1)$. (Received May 29, 1956.)

\section{R. M. Winger: On generalized Cotes'spirals.}

The curves considered have the polar equation $\rho=a \sec p \theta / q+k, p, q$ integer, $a, k$ real. The paper is the third of a trilogy by the present author concerning curves with analogous equations. The other cases were also discussed respectively by Moritz and Stratton, under the names cyclic-harmonic and polar tangent curves, employing mainly polar coordinates. Compact parametric equations are derived here, permitting the use of the technique for dealing with rational curves. The singularities and symmetry, together with the implied group properties, are studied. (Received July 5, 1956.)

\section{LOGIC AND FOUNDATIONS}

\section{5t. C. C. Chang: On the unions of chains of models.}

J. Łos and R. Suszko recently announced the following: I. An arithmetical class (for terminology and symbolism cf. Tarski, Indag. Math. vol. 16 (1954) pp. 572588) $K$ is determined solely by a set $\Sigma$ of sentences $\sigma$ each of which is logically equivalent to a sentence of the form (1) $\bigvee_{x_{1} \cdots x_{n}} \bigvee_{y_{1} \cdots y_{m}} \phi\left(x_{1}, \cdots, x_{n}, y_{1}, \cdots, y_{m}\right)$, where $\phi$ contains no quantifiers, if and only if $K$ is closed under unions of chains of models from $K$ (i.e., $U(K) \subset K$ ). (I) has been improved by the author to: II. If $K$ is an arithmetical class, then the arithmetical closure $L$ of the class $U(K)$ is also an arithmetical class and, furthermore, $L$ is determined by sentences of the form (1). It is clear that (II) is a stronger result than (I). It is also known that in (II) $L$ can not always be taken 
simply to be $U(K)$. The method used on proving (II) is essentially different from the method of Los and Suszko and consists in a mathematical characterization of classes $K$ (of models) which are arithmetical classes determined by sentences of the form (1). The above quoted result of $\operatorname{Los}$ and Suszko can be found in Bull. de l'Acad. Polonaise des Sciences, Classe III, vol. 3 (1955) pp. 201-202. (Received July 5, 1956.)

\section{6t. C. C. Chang: On arithmetical classes which are closed under} homomorphisms.

It is known that an arithmetical class $K$ of algebras (for terminology and symbolism see Tarski, Indag. Math. vol. 17 (1955) pp. 56-64) is closed under homomorphisms (i.e., $H(K) \subseteq K$ ) if the sentences $\sigma$ determining $K$ are all logically equivalent to sentences of the form (1) $Q_{\phi}$ where $Q$ is a sequence of quantifiers, $\phi$ contains no quantifiers, and $\phi$ is a conjunction of disjunctions of equalities. A sentence of form (1) is a universal (existential) positive sentence if $Q$ is a sequence of universal (existential) quantifiers. This abstract presents a partial solution to the converse of the above result. I. If $K$ is a universal (existential) class of algebras then the class $H(K)$ (the arithmetical closure of $H(K)$ ) is also a universal (existential) class and, furthermore, $H(K)$ (the closure of $H(K)$ ) is determined by universal (existential) positive sentences. II. If $K$ is a universal (existential) class, then $H(K) \subseteq K$ if and only if $K$ is determined by universal (existential) positive sentences. The proof depends on a mathematical characterization of universal and existential positive classes and the introduction of a notion of generalized subalgebras and congruence relations of an algebra. (Received July $5,1956$.

777. P. C. Gilmore: An interpretation for some set theories. Preliminary report.

Let $L$ be the first order theory with $e$ and $m$ as only primitive predicates and $(x)(y)(e(x, y) \supset m(x, y))$ as only axiom. Let $e(x)$ be $(u) m(u, x) \cdot(u)(v)((w)(e(w, u)$ $\equiv e(w, v)) \cdot e(u, x) \cdot \supset e(v, x)), x e y$ be $e(x, y) \cdot e(x) \cdot e(y), x \notin y$ be $\sim e(x, y) \cdot e(x) \cdot e(y)$, and $C(x)$ be $(E z)(e(x, z) \cdot(u)(e(u, z) \supset e(u)) \cdot(u)(v)(e(u, v) \cdot e(v, z) \cdot \supset e(u, z)))$. Interpretating $C(x)$ as the range of the variables of the New Foundations of Quine (NF), $x \in y$ as set membership and $x \notin y$ its complement, a translation $P^{\prime}$ in $L$ of any closed statement $P$ of NF can be constructed. Let LNF be $L$ with $P^{\prime}$ as added axiom for any $P$ which is the closure of a member of the Aussonderungs scheme (R3) of NF. Then it is shown that for any closed $P, P^{\prime}$ is provable in LNF if and only if $P$ is provable in NF. Similar results can be proved for several other first order set theories as well as for the monadic simple theory of types. In each case the extension of $L$ in which the set theory is interpreted is an extension by the addition as axioms of statements which can be interpreted as asserting the existence of certain objects. Since $e$ and $m$ can be interpreted as relations between concrete objects (abstract Journal of Symbolic Logic, June, 1956) several set theories can be interpreted in terms of concrete objects and relations between them. (Received June 25, 1956.)

\section{8t. Richard Montague: Models of set theories.}

For terminology see Abstract 738t. Theorem 1: If T is a set theory in which the axiom of extensionality is valid and which has a model of class $\mathbf{S}_{1}$ having the power $\boldsymbol{N}_{\alpha}$, then $T$ has a model, of class $\mathbf{S}_{2}$, of any power $\boldsymbol{\aleph}_{\beta} \leqq \boldsymbol{N}_{\alpha}$. Theorem 2. If $T$ is a set theory (e.g., $Z \mathbf{F}$ ) in which the axiom of extensionality is valid and which has a model of class $\mathbf{S}_{1}$, then $r_{1} T=r_{2} T$, and both are countable. The proofs of Theorems 1 and 2 depend on Theorem 
3 of Mostowski, Fund. Math. vol. 36 (1949) pp. 141-164, together with the generalized Löwenheim-Skolem Theorem. Assume the existence of $\vartheta$, the first strongly inaccessible ordinal $>\omega$. Theorem 3. $r_{3} Z F<\vartheta$. Thus $Z F$ does not guarantee (in the sense of models of class $S_{3}$ ) the existence of all sets of accessible rank. (Received July 6,1956.)

779. Steven Orey: Regular classes of relational systems. Preliminary report.

Let $(A ; \bar{R})=\left\langle A, R_{0}, \cdots, R_{\xi}, \cdots\right\rangle$ be a relational system in the sense of Tarski (for terminology see Tarski, Proc. Koninklijke Nederlandse Akad. van Wetenschappen, vol. 57, pp. 572-581) and let $F(\bar{R})$ be the union of the fields of $R_{0}, \cdots, R_{\xi}, \cdots$. If $C$ is a class of relational systems the regularization of $C, \widehat{C}$, is to consist of all $(A ; \bar{R})$ such that for some $B,(B ; \bar{R}) \in C$. It is proved that if $C$ is an arithmetic class so is $\widehat{C}$. On the other hand, for some higher order systems $L$ there are formulas $\phi$ (containing higher order bound quantifiers) corresponding to classes of relational systems $C$ (the models of $\phi$ ) such that $\widehat{C}$ does not correspond to any formula of $L$. (Received July 6 , 1956.)

780. R. M. Robinson: Restricted set-theoretical definitions in arithmetic.

It is known that addition of natural numbers is set-theoretically definable in terms of successor, but not arithmetically definable. A. Tarski has proposed consideration of an intermediate type of definition, in which variables ranging over natural numbers and variables ranging over sets of natural numbers are allowed, and has raised the question whether addition is definable in terms of successor in this sense. As a contribution to this problem, it is shown that addition $(x+y)$ is definable in terms of successor $(x+1)$ and double $(x+x)$. (Received May 23, 1956.)

\section{R. M. Smullyan: Elementary formal systems.}

Elementary formal systems are a simplification of Post's canonical languages; "productions" are replaced by the rules of substitution and detachment (modus ponens). Let $a_{1}, \cdots, a_{m}, x_{1}, \cdots, x_{t}, b_{1}, \cdots, b_{r}, \rightarrow$ be a collection of symbols. The $x_{i}$ are called "variables" (over the strings in the $a_{i}$ ); the $b_{i}$, "auxiliary letters"; the sign " $\rightarrow$ " is for logical implication. An atomic string is one which lacks " $\rightarrow$ ". An atomic string $F_{1}$, or a string $F_{1} \rightarrow F_{2} \rightarrow \cdots \rightarrow F_{m}$, where each $F_{i}$ is atomic, is a w.f.f. (well formed formula). A set $\mathfrak{F}$ of w.f.f.s $A_{i}, \cdots, A_{j}$ is an elementary formal system over the signs $a_{1}, \cdots, a_{m}$; the $A_{i}$ are the axioms of $\mathcal{F}$. A theorem of $\mathcal{F}$ is a string derivable from the axioms by rules R1-substitution of strings in the $a_{i}$ for variables; R2-inferring $X_{2}$ from $X_{1}$ and $X_{1} \rightarrow X_{2}$, providing $X_{1}$ is atomic. In $\mathcal{F}$, an auxiliary letter $b_{i}$ represents the set of all strings $X$ (in the $a_{i}$ ) such that $b_{i} X$ is a theorem of $\mathcal{F}$. Representability in these systems is provably equivalent to representability in Post's systems; this includes all "formal" or "finitary" languages. Elementary formal systems yield a very simple construction of an undecidable formal system - this leads readily to Gödel's incompleteness theorem. The proof of its undecidability is vastly simpler than Post's proof for the Universal Canonical system; Post's normal form theorem is completely circumvented. (The work in this paper was sponsored jointly by the Army, Navy and Air Force under contract with Massachusetts Institute of Technology. The author is a Staff Member, at Lincoln Laboratory, Massachusetts Institute of Technology. (Received July $5,1956$. ) 


\section{2t. R. M. Smullyan: On definability by recursion.}

A new approach to recursive enumerability is considered based on the notion of "minimal models." A formula of the lower functional calculus of the form $F_{1} \cdot F_{2} \ldots$ $F_{n-1} \cdot \supset \cdot F_{n}$ (or $F_{1}$ alone, if $n=1$ ) in which each $F_{i}$ is atomic, and $F_{n}$ contains no predicate constants, is termed regular. Let $A$ be a finite set of regular formulae; $\Sigma$ a collection of sets and relations, on some universe $U ; I$ an interpretation of the predicate constants (occurring in $A$ ) as elements of $\Sigma$. The ordered triple $\mathcal{L}$ viz. $(A, U, I)$ is a recursive logic over $\Sigma$. A model of $\mathcal{L}$ is an interpretation of the predicate variables $P_{i}$ in which each formula of $A$ is valid. Let $P_{i}^{*}$ be the intersection of all attributes assignable to $P_{i}$ in some model; these $P_{i}^{*}$ are called definable in $\mathcal{L}$. If each $P_{i}$ is interpreted as $P_{i}^{*}$, it can be proved that there is a model-this is the minimal model. Sets definable in some $\mathfrak{L}$ over $\Sigma$ are termed recursively definable from $\Sigma$. It is proved: (1) the recursively enumerable sets are precisely those which are recursively definable from the successor relation and the unit set $\{0\}$; (2) Post's canonical sets in an alphabet $a_{1}, \cdots, a_{n}$, are those recursively definable from the concatenation relation and the unit sets $\left\{a_{1}\right\}, \cdots,\left\{a_{n}\right\}$. (The work in this paper was sponsored jointly by the Army, Navy and Air Force under contract with Massachusetts Institute of Technology. The author is a Staff Member at Lincoln Laboratory, Massachusetts Institute of Technology.) (Received July 5, 1956.)

\section{3t. Alfred Tarski: Notions of proper models for set theories.}

A set theory $T$ is a theory with standard formalization (cf. Tarski-MostowskiRobinson, Undecidable theories, 1953) and with binary predicate " $\in$ " as the only nonlogical constant. General set theory, $S$, is based upon the axioms of extensionality, union, power set, pairs, and regularity, and the "Aussonderungsschema"; the axiom of choice may or may not be included. Zermelo set theor $y, Z$, has, in addition, the axiom of infinity. Zermelo-Fraenkel set theory, $Z \boldsymbol{F}$, has, further, the replacement schema. A model $\mathfrak{M}=\langle M, R\rangle$ of a set theory $\boldsymbol{T}$ is a proper model of the first class $\left(\mathfrak{M} \in \mathbf{S}_{1}\right)$ if $R=E_{M}$, i.e., $R=\{\langle x, y\rangle / x \in y$ and $x, y \in M\} ; \mathfrak{M} \in \mathrm{S}_{2}$ if, in addition, $x \in y \in M$ always implies $x \in M ; \mathfrak{M} \in \mathrm{S}_{3}$ if, further, $x \subseteq y \in M$ always implies $x \in M ; \mathfrak{M} \in \mathrm{S}_{4}$ if, for some ordinal $\alpha, M$ is of the form $\mathfrak{A}_{\alpha}=\left\langle R(\alpha), E_{R(\alpha)}\right\rangle$, where $R(\alpha)=\{x / \rho x<\alpha\}$ (cf. Tarski, Bull. Amer. Math. Soc. Abstract 61-5-628). The rank $r_{i} T$ of a set theory $T$ $(i=1,2,3,4)$ is the least ordinal $\alpha$ such that $\alpha=\rho M$ for some model $\langle M, R\rangle$ of $T$ of class $S_{i}$. Clearly, $r_{1} T \leqq r_{2} T \leqq r_{3} T \leqq r_{4} T ; r_{i} S=\omega$ and $r_{i} Z=\omega+\omega$ for $i=1,2,3,4$. For deeper results see Abstracts 778 and 784 of Montague and Vaught. (Received August 13, 1956.)

\section{4t. R. L. Vaught: On models of some strong set theories.}

For terminology see Abstracts 778 and 783. A set $A$ is definable in the model $\mathfrak{M}=\langle M, R\rangle$ of a set theory $\boldsymbol{T}$ if, for some formula $\phi$ of $\boldsymbol{T}$ with one free variable, $A$ is the unique member of $M$ satisfying $\phi$ in $\mathfrak{M}$. Theorem 1 . If $\mathfrak{A}_{\alpha}=\left\langle R(\alpha), E_{R(\alpha)}\right\rangle$ is a model of $\boldsymbol{Z F}$, and $\boldsymbol{T}_{\alpha}$ is the set theory whose valid sentences are all sentences true in $\mathfrak{N}_{\boldsymbol{\alpha}}$, then the union of all sets definable in $\mathfrak{A}_{\alpha}$ is equal to $R(\beta)$ for a certain $\beta \leqq \alpha, \beta$ is confinal with $\omega$, and $A_{\beta}$ is a model of $T_{\alpha}$ (indeed, $A_{\alpha}$ is an "arithmetical extension" of $A_{\beta}-\mathrm{cf}$. Tarski-Vaught, Arithmetical extensions of relational systems, to appear in Comp. Math.). The proof uses a device employed previously by Montague (Bull. Amer. Math. Soc. Abstract 61-2-344). Corollary: $r_{1} Z F<r_{4} T_{\vartheta}<\vartheta$. (That $r_{4} Z F<\vartheta$ also follows from an earlier result of Montague (Theorem 3 of Abstract 778) and the Lemma: If $\mathfrak{M} \in \mathrm{S}_{3}$ and $\mathfrak{M}$ is a model of $\boldsymbol{Z F}$, then $\mathfrak{M} \in \mathrm{S}_{4}$.) Thus neither $\boldsymbol{Z} \boldsymbol{F}$ nor $\boldsymbol{T}_{\vartheta}$ insures (in 
the sense of models of class $\mathbf{S}_{4}$ ) the existence of all sets of accessible rank. (Received August 13, 1956.)

\section{Statistics and Probability}

785t. G. A. Baker, Jr. and G. A. Baker, Sr.: Peculiar speeds of stars.

For the case of the single drift hypothesis of the distributions of velocities where the three space components of each of the observed stars are known without error and the three components of the velocity of the "local standard of rest" are known exactly, the distribution of the peculiar speeds is easily written down and is seen to depend on a single parameter. This case and successive generalizations about the local standard of rest, fundamental character of the distributions of space velocities, and knowledge of parameters are treated in this paper. The principal tools are modified maximum likelihood estimation, moment-generating functions, Laplace transforms, and expected values. The results consist of frequency distributions of peculiar speeds of stars under stated assumptions, confidence limits for estimates of parameters, and the effect of lack of exact knowledge of the local standard of rest on the distribution of the peculiar speeds of stars. (Received June 4, 1956.)

786. G. E. Baxter: Some conditional probability distribution functions.

Let $\{x(t), 0<t \leqq \infty\}$ be a separable stochastic process with stationary, independent increments for which $x(0)=0$. Then for any $\sigma>0(s, \lambda>0)$ define $N(\alpha, T, \sigma)$ by (1) $N(0, T, \sigma)=0$ where $0<T<\infty, \quad$ (2) $\int_{0}^{\infty} \int_{0}^{\infty} e^{-\lambda \alpha-s T} d_{\alpha} N(\alpha, T, \sigma) d T$ $=\exp \left[\int_{0}^{\infty} \int_{0}^{\infty}\left(e^{-\lambda \alpha-s T}-e^{\sigma T}\right) / T d_{\alpha} P\{x(T)<\alpha\} d T\right]-\exp \left[\int_{0}^{\infty} e^{-\sigma T} / T P\{x(T) \geqq 0\} d T\right]$. Then, for any $\alpha \geqq 0$, the conditional probability distribution function $P\{x(T)<\alpha \mid x(t)$ $\geqq 0$ for all $0 \leqq t \leqq T\}=N(\alpha, T, \sigma) / N(\infty, T, \sigma)$. In the special case $P\{x(T) \geqq 0\}=1 / 2$, it follows from this and the limiting form of Spitzer's identity (Bull. Amer. Math. Soc. Abstract 61-6-819) that $\int_{0}^{\infty} e^{-s T} N(\alpha, T, \sigma) d T=(\sigma s)^{1 / 2} \int_{0}^{\infty} e^{-s T} P\left\{\sup _{0<t \leqq} x(t)<\alpha\right\} d T$. A formula is also found for the distribution $P\{x(T) \leqq \alpha \mid x(t)>0$ for all $0<t \leqq T\}$, and calculations are made for certain special cases. (Received July 19, 1956.)

\section{7t. A. T. Bharucha-Reid: On random elements in Orlicz spaces. Preliminary report.}

Let $(\Omega, Q, p)$ be a probability space, and let $x=x(\omega)$ be a function on $\Omega$ to $X . x(\omega)$ is called a random element in $X$. In this note we begin the study of random elements in Orlicz spaces, that is when $X=L_{\Phi}(\Omega, p)$. The expectation of $x, m=E x$, is defined by the Pettis integral, and is that element of $X$ such that for all $x^{*} \in X^{*}, x^{*}(m)$ $=\int_{\Omega} x^{*}(x(\omega)) d p$, where $x^{*}(x)=\int_{\Omega} x(\omega) y(\omega) d p$ is a bounded linear functional on $X$ to $x^{*}$, and $y \in L_{\Psi}$ (the conjugate space) is a uniquely determined random element. We consider in particular laws of large numbers for random elements in Orlicz spaces. Since $L_{\Phi}$ contains the same random elements as $L_{p}$ when $\Phi(s)=\alpha s^{p}, \alpha>0,1 \leqq p<\infty$, these theorems yield laws of large numbers for random elements in $L_{p}$ spaces as a special case. (Received June 4, 1956.)

788t. Aryeh Dvoretzky: Almost sure everywhere divergence of random series.

It is well known that if $a_{n}(n=0,1,2, \cdots)$ is a sequence of complex numbers and $r_{n}(t)(n=0,1,2, \cdots)$ denote the Rademacher functions then the series $\sum_{n=0}^{\infty} r_{n}(t) a_{n}$ 
diverges for almost all $t$ (in the usual Lebesgue sense) if and only if $\sum_{n=0}^{\infty}\left|a_{n}\right|^{2}$ is divergent. It follows immediately, by Fubini's theorem, that if $\sum_{n=0}^{\infty}\left|a_{n}\right|^{n=0}+\infty$, then for almost all $t$ the series $\sum_{n=0}^{\infty} r_{n}(t) a_{n} z^{n}$ diverges almost everywhere on the circle $|z|=1$ (again in the $(n=0,1,2, \cdots))$ is monotone and $\left|a_{n}\right|^{5+\epsilon}=\infty$ for some $\epsilon>0$ then the italicized almost in the preceding statement may be dropped; i.e. under the conditions $\sum_{n=0}^{\infty} r_{n}(t) a_{n} z^{n}$ is, for almost all $t$, nowhere convergent on $|z|=1$. Similar results obtain for other types of random power series as well as for trigonometric series, Dirichlet series, etc. (Received July 3, 1956.)

789. L. R. Ford, Jr.: Solution of a ranking problem from paired comparisons.

Given a square matrix $A=\left(a_{i j}\right)$, with $a_{i j} \geqq 0, a_{i i}=0$, where $a_{i j}$ is interpreted as the number of times $i$ has been preferred to $j$, weights are assigned to each index by a maximum likelihood method. The ratio $w_{i}: w_{j}$ is interpreted as the odds in a single $i j$ comparison, and the $w_{i}$ are determined to maximize the a priori probabilityof $A$. Under a weak restriction on $A$, existence and uniqueness of the maximizing $w$ are shown in the region $\left\{w_{i}>0 ; \sum w_{i}=1\right\}$; an iterative scheme for computing $w$ is given and shown to converge to the desired solution. (Received June 28, 1956.)

\section{L. L. Helms: A mean martingale convergence theorem.}

Let $(E, \mathfrak{B}, \mu)$ be a measure space consisting of a set $E$, a $\sigma$-algebra $\mathfrak{B}$ of subsets of $E$, and a finite measure $\mu$ defined on $\mathscr{B}$, and let $(A,>)$ be an arbitrary directed set. $L^{p}, 1 \leqq p<\infty$, will denote the totality of functions which are measurable relative to $\mathfrak{B}$ and $p$ th power integrable with respect to $\mu$. A net $\left\{x_{a}, a \in A\right\} \subset L^{1}$ is called a martingale if there is a monotone increasing net $\left\{\mathfrak{B}_{a}, a \in A\right\}$ of $\sigma$-subalgebras of $\mathfrak{B}$ such that $x_{a}$ is measurable relative to $\mathfrak{B}_{a}$ and $\int_{S} x_{a} d \mu=\int_{S} x_{b} d \mu$ for $S \in \mathfrak{B}_{a}$ and $b>a$. The directed set $\left(A_{\infty},>\right)$ is obtained from $(A,>)$ by adjoining an ideal element " $\infty$ " to $A$ and defining $\infty>a$ for all $a \in A_{\infty}=A \cup\{\infty\}$. The author shows that the following four properties of a martingale $\left\{x_{a}, a \in A\right\} \subset L^{p}, 1 \leqq p<\infty$, are equivalent: (i) the net $\left\{x_{a}, a \in A\right\}$ is uniformly integrable if $p=1$ or strongly bounded if $p>1$. (ii) the net $\left\{x_{a}, a \in A\right\}$ converges weakly in $L^{p}$; (iii) there is an $x_{\infty} \in L^{p}$ such that the net $\left\{x_{a}, a \in A_{\infty}\right\}$ is a martingale; and (iv) the net $\left\{x_{a}, a \in A\right\}$ converges strongly in $L^{p}$. (Received June 15, 1956.)

791. Samuel Karlin and J. L. McGregor (p): Coincidence probabilities. Preliminary report.

Let $P(t)=\left(P_{i j}(t)\right), t \geqq 0$, be the transition matrix of a Markoff process whose state space is a finite or infinite set of consecutive integers. The path functions are assumed to be continuous, i.e., a transition from $i$ to $j$ cannot occur without visiting all intervening states. Let $\Delta$ denote the $n$-square determinant formed by the rows of $P(t)$ with indices $i_{1}<i_{2}<\cdots<i_{n}$ and the columns with indices $j_{1}<j_{2}<\cdots<j_{n}$. It is shown that $\Delta$ is the probability that if $n$ particles start at $t=0$ at $i_{1}, \cdots, i_{n}$, then at time $t$ they are at $j_{1}, \cdots, j_{n}$ without any two of them ever having been coincident. The analogous but more involved result when the path functions are not necessarily continuous is also obtained. (Received July 2, 1956.)

\section{E. G. Kimme: A general convergence theorem for sequences of stochastic processes.}

It has been shown that if $\left\{x_{n}(t, \omega), t \in[0,1], n \geqq 1\right\}$ is a sequence of separable 
stochastic processes with independent increments converging uniformly in distribution to separable stochastic process with independent increments and no fixed points of discontinuity $\{x(t, \omega), t \in[0,1]\}$, then for a large class of functionals $F[]$ defined on the sample functions of these processes, $F\left[x_{n}(\cdot,(\omega)]\right.$ converges in distribution to $F[x(\cdot, \omega)]$. The present paper removes the conditions of independence from the stated result. (Received June $28,1956$. )

\section{Klaus Krickeberg: Stochastic convergence of semimartingales.}

Let $\mathfrak{B}$ be a Boolean sigma-algebra with unit $E$ bearing a strictly positive and finite measure $\mu$, and $\mathfrak{F}$ the space of all $\mathfrak{B}$-measurable extended real-valued functions on $E$ (functionoids, "Ortsfunktionen), $\mathfrak{B}$ and $\mathfrak{F}$ being complete lattices under their order relations $\leqq$. Given a Moore-Smith sequence $\left(f_{\sigma} ; \sigma \in \Theta\right)$ with $f_{\sigma} \in \mathfrak{F}$ its stochastic limit superior, $s \lim \sup _{\sigma} f_{\sigma}$, is defined as the infimum of all $h$ in $\mathfrak{F}$ such that $\lim _{\sigma} \mu\left\{h^{\prime}<f_{\sigma}\right\}=0$ for every $h^{\prime}$ in $\mathfrak{F}$ with $h \leqq h^{\prime}$ and $\left\{h^{\prime}=h\right\}=\{h=+\infty\}$. Likewise $s \lim \inf _{\sigma} f_{\sigma}$ is obtained. Both limits are independent of $\mu$ and have the following properties: $s \lim \inf _{\sigma} f_{\sigma} \leqq s \lim \sup _{\sigma} f_{\sigma}$, and equality holds if and only if $\left(f_{\sigma}\right)$ converges stochastically; if each $f_{\sigma}$ is measurable relative to a fixed sigma-subalgebra $\mathfrak{A}$ of $\mathfrak{B}$, the stochastic limits are $\mathfrak{Q}$-measurable, too; $s \lim \sup _{\sigma} f_{\sigma} \leqq \lim \sup _{\sigma} f_{\sigma}$, where lim sup denotes the "order" limit superior in $\mathfrak{F}$; Fatou's lemma lim $\sup _{\sigma} \int_{E} f_{\sigma} d \mu \leqq \int_{E}\left(s \lim \sup _{\sigma}\right.$ $\left.f_{\sigma}\right) d \mu$ holds if $\left(f_{\sigma}\right)$ is terminally uniformly integrable. With the help of these stochastic limits inferior and superior it can be shown that each martingale $\left(f_{\sigma} ; \sigma \in \Theta\right)$ relative to an increasing Moore-Smith sequence $\left(\mathfrak{B}_{\sigma} ; \sigma \in \Theta\right)$ of sigma-subalgebras of $\mathfrak{B}$ converges stochastically if the positive parts $f_{\sigma}^{+}$or the negative parts $f_{\sigma}^{-}$have bounded expectations. Under similar assumptions any semimartingale relative to $\left(\mathfrak{B}_{\sigma}\right)$ is stochastically convergent, too. (Received July 5, 1956.)

794. R. B. Leipnik: Moment generating functions of quadratic forms in serially correlated normal variables.

An integral equation method used by Kac in the study of Wiener functionals is successfully adapted to the problem of calculating in closed algebraic form joint moment generating functions of linear combinations of four special quadratic forms in normal variables. The class of gaussian processes for which this method works is characterized. The autocovariance function is either of the form $\sigma^{2} \rho^{\left|t_{2}-t_{1}\right|}+\rho^{t_{1}+t_{2}}$ $\cdot\left(A\left(t_{1}\right)+A\left(t_{2}\right)\right)$ or $\sigma^{2} \min \left(t_{1}, t_{2}\right)+A\left(t_{1}\right)+A\left(t_{2}\right)$. The integral equation is solved with the help of Mehler's identity. For the case of the Uhlenbeck-Ornstein process $(A(t)=0)$ the moment generating functions are studied for various quadratic estimators of $\sigma^{2}$ and $\rho$. Asymptotic results as the number of observations $\rightarrow \infty$, and time between observations $\rightarrow 0$ are obtained. (Received June 28, 1956.)

\section{5t. George Marsaglia: Regression for independent variables.}

Consider random variables $x_{1}, \cdots, x_{n}, y_{1}, \cdots, y_{n}$ and assumptions of the type $E\left(y_{i}\right)=a E\left(x_{i}\right)+b$. For normal variables, this may be viewed as an extension of the general linear hypothesis, in that the vector of expected values is assumed to lie in the union of a collection of vector spaces, rather than in a single specified vector space. This weaker restriction on the expected values requires a more stringent ccndition on the covariance matrix, to ensure the existence of the maximum likelihood estimates The principal result of this note provides such estimates for the case of independent $x$ 's and $y$ 's, allowing multiple determinations. An incidental result is that the estimates of $a$ and $b$ provide, in terms of line fitting, the line which minimizes the sum of the squares of the distances from the points to the line. (Received July 23, 1956.) 


\section{TOPOLOGY}

\section{L. W. Anderson: On bounded subsets of topological lattices.}

A topological lattice is a Hausdorff space, $L$, together with a pair of continuous functions, $\wedge: L \times L \rightarrow L$ and $\bigvee: L \times L \rightarrow L$, which satisfy the usual conditions stipulated for a lattice. If $L$ is a locally compact connected topological lattice which is homeomorphic with a subset of Euclidean 2-space and if $C$ is a compact subset of $L$ then there are elements, $a$ and $b$, in $L$ such that $a \leqq x \leqq b$ for all $x \in C$. (Received July 5 , 1956.)

797. R. D. Anderson: One-dimensional continuous curves and a homogeneity theorem.

Let $\Omega$ be the class of all one-dimensional locally connected compact metric continua up to topological equivalence. Let $\mathfrak{M}$ be the subclass of $\Omega$ consisting of those elements of $\Omega$ without local cut points. The following three theorems are proved. I. In order that an element $M$ of $\mathfrak{M}$ be the universal curve it is necessary and sufficient that no open subset of $M$ be imbeddable in the plane. II. The simple closed curve and the universal curve are the only homogeneous elements of $\Omega$. III. In order that an element $K$ of $\Omega$ be the universal curve it is necessary and sufficient that $K$ be crossconnected. A continuum $X$ is said to be weakly cross-connected provided that for every two disjoint pairs $\left(A, A^{\prime}\right)$ and $\left(B, B^{\prime}\right)$ of points of $X$ there exist two disjoint arcs in $C$ containing $A+A^{\prime}$ and $B+B^{\prime}$ respectively. A locally connected continuum $X$ is said to be cross-connected provided that for every connected open subset $D$ of $X$ for which $\mathrm{Cl}(D)$ is locally connected, $\mathrm{Cl}(D)$ is weakly cross-connected. (Received June 29, 1956.)

\section{8t. R. D. Anderson: Zero-dimensional compact groups of homeo- morphisms.}

The author shows that each zero-dimensional compact metric topological transformation group can operate on a universal curve in a fixed point free manner, i.e. so that each element of the group other than the identity moves every point of the space (see the author's other abstract, this meeting, for some characterizations of the universal curve). Furthermore the space of orbits may be required to be a universal curve itself or, in case the group is infinite, the space of orbits may be required to be a regular curve. In the course of the proof of the above theorems it is also shown that for each finite group $G$ there exists an orientable 2-manifold $M(G)$ such that $G$ can operate on $M(G)$ in a fixed point free manner. (Received June 29,1956.)

\section{R. H. Bing: A necessary and sufficient condition that a 3-mani-} fold be $S^{3}$.

It is shown that a compact 3-manifold $M$ is topologically $S^{3}$ if each simple closed $J$ in $M$ lies in a topological cube $K$ in $M$. The outline of the proof is as follows. First, $M$ is triangulated so as to give it an affine structure. Next it is shown that $M$ is topologically $S^{3}$ if the 1 -skeleton $R_{1}$ of this triangulation lies on the interior of a polyhedral cube. Then it is shown that if $J$ is a polyhedral simple closed curve in $M$, then $J$ lies on the interior of a polyhedral cube in $M$. Finally a polyhedral simple closed curve $J$ is knotted about $R_{1}$ in such a way that any polyhedral cube that contains $J$ on its interior can be adjusted so as to contain $R_{1}$ on its interior. (Received June 29,1956 .) 


\section{0t. R. E. Chamberlin: The fixed point property for certain con- tinua.}

A tree is a finite, acyclic 1-dimensional complex. A tree-like continuum is a compact metric space with a cofinal sequence of open coverings whose nerves are trees. $X$ has the fixed point property if for every continuous $F: X \rightarrow X$ there is a $p$ with $F(p)=p$. Theorem: Every tree-like continuum has the fixed point property. If $T$ is a tree and $p$ and $q$ are points of $T$ let $[p, q]$ denote the smallest connected set containing $p$ and $q$. Definition: If $f: T \rightarrow T^{\prime}, g: T \rightarrow T^{\prime}$ are two simplicial mappings of a tree $T$ into a tree $T^{\prime}$, $f$ dominates $g$ if (a) $f$ is onto and (b) for every $p_{1}, p_{2} \in T$, there exist $q_{1}, q_{2} \in T$ with $f\left(q_{1}\right)=g\left(p_{1}\right), f\left(q_{2}\right)=g\left(p_{2}\right)$ and such that $f\left(\left[q_{1}, q_{2}\right]\right) \subset g\left(\left[p_{1}, p_{2}\right]\right)$. The proof of the Theorem depends on the Lemma: Given simplicial mappings $f, g: T \rightarrow T^{\prime}$ such that $f$ dominates $g$. Then there is a $p$ with $f(p)=g(p)$. Suppose $F: X \rightarrow X$ is continuous and $\left\{U_{i}\right\}$ and $\left\{V_{j}\right\}$ are finite open coverings of $X$ with nerves $T$ and $T^{\prime}$ respectively. If for each $i$ there is a $j$ with $F\left(U_{i}\right) \subset V_{j}$, the simplicial mapping $f: T \rightarrow T^{\prime}$ defined by assigning the vertex corresponding to $U_{i}$ to the vertex corresponding to some $V_{j}$ with $F\left(U_{i}\right) \subset V_{j}$ is said to be induced by $F$. Let $F: X \rightarrow X$ be the identity and $G: X \rightarrow X$ be continuous. If $X$ is tree-like, there is a cofinal sequence of open coverings with nerves $\left\{T_{n}\right\}$ and mappings $f_{n}, g_{n}: T_{n+1} \rightarrow T_{n}$ induced by $F$ and $G$ respectively with $f_{n}$ dominating $g_{n}$. This fact, together with the Lemma proves the Theorem. (Received July $5,1956$.

\section{Haskell Cohen and L. I. Wade: Clans with zero on an interval.}

A clan is a compact connected Hausdorff topological semigroup with unit. Let $S$ be a clan with zero on an interval. It is known that the unit 1 is an endpoint. Let $d$ be the other endpoint, $L$ be the closed interval $[d, 0]$, and $R$ be the closed interval $[0,1]$. It can be shown that $R$ is an abelian subclan, and its structure has been characterized in an unpublished work of P. S. Mostert and A. L. Shields. It is now shown that (1) $R L$ and $L R$ are contained in $L$, (2) either $L^{2} C L$ or $L^{2} C R$, (3) $L$ is abelian. Also, under the additional hypothesis that $\left\{1 \mid 1 \in L\right.$ and $\left.1^{2}=d^{2}\right\}=d$, it follows that (4) $S$ is abelian, (5) if $L^{2} C L$ then $L$ is a homomorphic image of an ideal in $R$, (6) if $L^{2} \subset R$ then $L$ is a copy of an ideal in $R$ with its products in $R$ as in the interval from $-1 / 2$ to 1 under ordinary multiplication. (Received July 6,1956 .)

\section{Leonard Gillman: Rings with Hausdorff structure space. I.}

Let $A=\{\cdots, x, \cdots\}$ be semi-simple, $\Re=\{\cdots, P, \cdots\}$ its primitive ideals, $\mathfrak{Q}=\{\cdots, Q, \cdots\}$ its proper prime ideals, and let $\mathfrak{S}=\{\cdots, S, \cdots\}$ satisfy $\mathfrak{B} \subset \mathfrak{S} \subset \mathfrak{Q}$. Define $\mathfrak{S}(x)=\{S: x \in S\}$; the sets $\mathfrak{S}(x)$ are a base for the closed sets in $\mathfrak{S}$. Define $N_{S}=\{x: \exists$ nbhd $\mathfrak{u}$ of $S$ in $\mathfrak{S}$ such that $x \in \cap \mathfrak{U}\} ; N_{S}$, is an ideal and $N_{S} \subset S$. 1. If $S \supset Q$, then $Q \supset N_{S}$. 2. (Due to C. W. Kohls.) Every $N_{S}$ is an $\bigcap$ of $Q$ 's 3. Every $\mathfrak{S}(x)$ is open iff every $N_{S}=S$. 4 . These are equivalent: (a) $\subseteq$ is $H$ (=Hausdorff); (b) given $S \neq S^{\prime}, \exists x$ such that $x \in N_{S}, x \in S^{\prime}$; (c) each $S$ is the unique $S^{\prime}$ such that $S^{\prime} \supset N_{S}$. 5. If every $\subseteq(x)$ is open, then $\subseteq$ is $H$. 6. If $\subseteq$ is $H$, then each $Q \subset$ at most one $S$. 7. Corollary. If $A$ is an integral domain with at least two $P$ 's, then $\mathfrak{B}$ is not $H$. 8. If $\subseteq$ is $H$, then these are equivalent: (a) $\mathfrak{Q}=\subseteq$; (b) every $Q$ is an $\bigcap$ of $S$ 's; (c) every $Q \subset$ an $S$, and every $\mathfrak{S}(x)$ is open. 9. Corollary. $\mathfrak{Q}$ is $H$ iff every $\mathfrak{Q}(x)$ is open. 10. Corollary. If $A$ is biregular, then $\mathfrak{Q}=\mathfrak{B}$, and every $\mathfrak{B}(x)$ is open. 11 . A is strongly regular iff (i) every $A / P$ is a division ring, (ii) every $\mathfrak{B}(x)$ is open, and (iii) every $A / \cap \sim \mathfrak{P}(x)$ has an identity. (Received July $6,1956$. 


\section{3t. Leonard Gillman: Rings with Hausdorff structure space. II.}

Notation as in preceding abstract. 12. If $A$ is a commutative ring with identity and $\subseteq$ is $H$, and if every finitely generated ideal is principal, then every $N_{S}$ is prime. 13. If $A$ is a commutative ring with identity and $\mathfrak{P}$ is $H$, then these are equivalent: (a) $A$ is regular; (b) every ideal is an $\bigcap$ of $P^{\prime}$ s; (c) every $Q$ is an $\bigcap$ of $P$ 's; (d) $\mathscr{Q}=\mathfrak{B}$; (e) $A$ is adequate: (f) every $\mathfrak{B}(x)$ is open. (The example $A=$ ring of integers shows that these not be equivalent if $\mathfrak{B}$ is not $H$.) The proof of 12 makes use of the following general results. 14 . For any family $\mathfrak{A}$ of prime ideals, these are equivalent: (a) for all $\mathfrak{B} \subset \mathfrak{A}, \cap \mathfrak{Q}$ is prime; (b) for any $Q, Q^{\prime} \in \mathfrak{A}, Q \cap Q^{\prime}$ is prime; (c) $\mathfrak{A}$ is totally ordered under set inclusion. 15. Let $A$ be a commutative ring with identity, let $\mathfrak{A}$ denote the family of all prime ideals contained in a given proper ideal $I$, and suppose that every finitely generated ideal contained in $I$ is principal. Then $\mathfrak{A}$ is totally ordered under set inclusion. (Received July 6, 1956.)

804. Melvin Henriksen: On minimal completely regular spaces associated with a given ring of continuous functions.

Let $C(X)$ denote the ring of continuous real-valued functions on a completely regular space $X$. If $X$ and $Y$ are completely regular spaces, the rings $C(Y)$ and $C(X)$ are said to be strictly isomorphic if one is a dense subspace of the other, say $Y$ is dense in $X$, and every $f \in C(Y)$ has a continuous extension over $X$. In an unpublished paper (see Bulletin Amer. Math. Soc. Abstract 62-4-545) L. J. Heider asked (in different but equivalent language) if it is possible to associate with each completely regular space $X$ a subspace $\mu X$ minimal with respect to the property that $C(\mu X)$ and $C(X)$ are strictly isomorphic. Let $\eta X=\{p \in X: C(X \sim\{p\})$ and $C(X)$ are not strictly isomorphic $\}$. Theorem. A minimal subspace $\mu X$ exists if $C(\eta X)$ and $C(X)$ are strictly isomorphic, in which case $\mu X=\eta X$. Thus if $\mu X$ exists, it is unique. Finally it is noted that if $\beta N$ denotes the Stone-Čech compactification of the countable discrete space, then $\mu(\beta N)$ does not exist. (Received July 6, 1956.)

805t. R. P. Hunter: Types of $(n, k)$ adherence and indecomposability.

The connexe $M$ is said to contain an $(n, k)$ adherent sequence of sets $P_{1}, P_{2}, \cdots$, $P_{n+1}$, if $M-P_{1} \supset M_{1}-P_{2} \supset \ldots \supset M_{n-1}-P_{n} \supset M_{n} \supset V \supset P_{n+1}$; where each $M_{i}$ is a proper connexe subclosure of, and relatively closed in $M_{i-1}, M=M_{0}, P_{i} \cdot M_{i-1} \# 0$ and $V$ is open in $M_{k}$. Definitions of Swingle are used (Bull. Amer. Math. Soc. vol. 47 (1941) p. 796). The following hold in a Moore space $l_{3}$. The connexe $M$ is indeco mposable if and only if it contains no $(1,0)$ adherent sequence. Every proper connexe subclosure of $M$ is indecomposable if and only if $M$ contains no $(2,1)$ adherent sequence. If the compact connexe $M$ contains a $(1,0)$ but no $(2,1)$ adherent sequence it is nonclosed. The connexe $M$ is $n$-indecomposable if and only if it contains $n$ but not more than $n$ points $p_{i} i=1,2, \cdots, n$ such that $p_{i}, p_{j}$ is a $(1,0)$ adherent sequence. If the closed connexe $M$ is the essential sum of $n$ proper connexe subclosures and contains no $(n, n-1)$ adherent sequence it is $n$-indecomposable and indeed the essential sum of $n$ non-compact hereditarily indecomposable continua. (Received July 2,1956.)

806. V. L. Klee, Jr.: Homogeneity of infinite-dimensional parallelotopes.

A topological space $X$ is said to be homogeneous with respect to a family $S$ of its subsets if whenever $Y \in S$ and $f$ is a homeomorphism of $Y$ onto a set $f Y \in S$, then $f$ 
can be extended to a homeomorphism of $X$ onto $X$. It is known that Hilbert space is homogeneous with respect to its compact subsets, and the Hilbert parallelotope $P$ with respect to its finite subsets [Trans. Amer. Math. Soc. vol. 78 (1955) pp. 30-45]. The scope of the latter result is extended by the fact that every infinite-dimensional compact convex subset of a normed linear space is homeomorphic with $P$ [ibid.]. The present paper employs earlier methods, and especially the transformations of $\mathrm{O}$. $\mathrm{H}$. Keller [Math. Ann. vol. 105 (1931) pp. 748-758], to establish homogeneity of $P$ with respect to its countable closed subsets. It is also proved that there is only one isotopyclass of homeomorphisms of $P$ onto $P$, a result established earlier for Hilbert space [Trans. Amer. Math. Soc. vol. 74 (1953) pp. 10-43]. (Received July 3, 1956.)

\section{7t. W. S. Massey: Some cohomology invariants of higher kind.}

The object of this paper is to give some examples of cohomology operations which are homotopy type invariants of topological spaces, but are not universally defined. They are constructed by purely algebraic methods starting from a suitable (e.g. singular or Alexander-Spanier) cochain ring of the given space $X$ with coefficients in an associative ring $R$. From an ordered abstract simplicial complex $K$ and the given cochain ring one constructs (by a process which is too involved to describe here) a bi-complex. As usual, this bi-complex gives rise to two spectral sequences, only one of which is of interest. The first term $E_{1}$ of the interesting spectral sequence is a direct sum of tensor products of the cohomology ring $H^{*}(X, R)$ with itself, and the differential operator $d_{1}: E_{1} \rightarrow E_{1}$ may be expressed in terms of the products in $H^{*}(X, R)$. The succeeding differential operators, $d_{2}, d_{3}$, etc., are the desired cohomology operations of higher kind. For different choices of the abstract complex $K$, one obtains different spectral sequences. If $K$ consists of a single $m$-simplex and all its faces, it is possible to make explicit computations and give examples where these higher operations distinguish between spaces of different homotopy types. (Received April 9, 1956.)

\section{P. S. Mostert: On a compact Lie group acting on a manifold.}

Let $M$ be a manifold of dimension $n+1$ and $G$ a compact Lie group acting on $M$ in such a way that there is at least one $n$-dimensional orbit. Then the space of orbits $M / G$ is homeomorphic to one of (1) a circle, (2) an open interval, (3) a half-open interval, or (4) a closed interval. Moreover, there exists a subgroup $N$ of $G$ such that, in (1) and (2), $M$ is homeomorphic to $M / G \times G / N$; in case (3), there exists a subgroup $K \supset N$ such that $K / N$ is an $r$-sphere for some $r \geqq 0$ and such that $M$ is homeomorphic to $(M / G \times G / N) / R$ where $R$ is the relation identifying $p \times G / N$ to $p \times G / K$ over the end point $p$ of $M / G$; in (4) a similar situation prevails with subgroups $K_{1}$ and $K_{2}$. (In this latter case, $K_{1} / N$ and $K_{2} / N$ may be of different dimensions). The action of $G$ is equivalent to $G$ acting differentiably. Applications are given in dimensions two and three. (Received June 28, 1956.)

\section{9t. J. C. Oxtoby: A category analogue of the zero-one law.}

Let $X$ be the product of a sequence of topological spaces $X_{i}$, each of which has a countable base and in each of which every nonempty open set is of second category. Then $X$ has the same two properties and every tail set in $X$ that has the property of Baire is either of first category or residual. (A tail set is a set of the form $\left(P_{i=1}^{n} X_{i}\right) \times B_{n}$ for every $n$.) The proof is based on the Kuratowski-Ulam category analogue of the Fubini Theorem. (Received August 21, 1956.) 
810t. C. D. Papakyriakopoulos: On the ends of the fundamental groups of 3-manifolds with boundary.

Let $M$ be a finite 3-manifold closed (=without boundary) or with boundary whose components are not 2 -spheres. When is the number e of ends of $\pi_{1}(M), 0$ or 1 or 2 or $\infty$ ? This problem has been solved if $M$ is closed, by E. Specker [Commt. Math. Helv. vol. 23 (1949) Satz VI]. If $M$ has boundary the solution is as follows: (1) If $M$ is aspherical and the injection $\pi_{1}\left(N_{i}\right) \rightarrow \pi_{1}(M)$ is an isomorphism for every component $N_{i}$ of $N$, then $e=1$. (2) If $M$ is aspherical, connected and of genus one, and the injection $\pi_{1}(N) \rightarrow \pi_{1}(M)$ is not an isomorphism, then $e=2$. (3) In any other case, $e=\infty$. The proof of (1)-(3) is based on the theorem contained in the author's abstract On a lemma of Kneser [Bull. Amer. Math. Soc. Abstract 62-2-279], on two theorems of the author [Ann. Math. vol. 62, no. 2 (1955) Theorems 1 and 2], and on a theorem of E. Specker [Satz VII, of the above paper]. (Received May 3, 1956.)

\section{L. E. Pursell: Abstract multi-derivatives on infinitely-differenti- able manifolds.}

Let $X$ be an $\infty$-differentiable manifold. An abstract derivative is a mapping $L: C^{\infty}(X) \rightarrow C^{\infty}(X)$ such that $L(a f+b g)=a L f+b L g$ and $L(f g)=f L g+g L f$. An abstract multi-derivative of order $r$ is a mapping $T_{r}:\left[C^{\infty}(X)\right]^{r} \rightarrow C^{\infty}(X)$ which is an abstract derivative in each of its independent variables when the other $r-1$ variables are held constant. It is shown that on a coordinate neighborhood where $x^{i_{1}}, \cdots, x^{i_{r}}$ are the coordinate functions $T_{r}\left(f_{1}, \cdots, f_{r}\right)=\sum_{i_{1} \ldots i_{r}} \cdot\left(\partial f_{1} / \partial x^{i_{1}}\right) \cdots\left(\partial f_{r} / \partial x_{r}^{i_{r}}\right) T_{r}\left(x^{i_{1}}, \cdots\right.$, $x^{i_{r}}$. Hence abstract multi-derivatives may be identified with contravariant tensor fields. (C. Chevalley, Theory of Lie groups, I, has a similar result for analytic manifolds.) For abstract multi-derivatives $S_{m}, T_{n}$ we define the Kronecker or outer product $S_{m} T_{n}$ by $\left(S_{m} T_{n}\right)\left(f_{1}, \cdots, f_{m+n}\right)=S_{m}\left(f_{1}, \cdots, f_{m}\right) T_{n}\left(f_{m+1}, \cdots, f_{m+n}\right)$. Consider sequences $\left\{S_{r}\right\}_{r=0}^{\infty},\left\{T_{r}\right\}_{r=0}^{\infty}$ whose 0 th element is a real number and whose $r$ th element is a multi-derivative of order $r$. Define $\left\{S_{r}\right\}+\left\{T_{r}\right\}=\left\{S_{r}+T_{r}\right\}$ and $\left\{S_{r}\right\}\left\{T_{r}\right\}$ $=\left\{S_{0} T_{r}+S_{1} T_{r-1}+\cdots+S_{r} T_{0}\right\}$. These sequences form a ring which determines the space $X$ if $X$ is compact. For noncompact $X, X$ is determined if one considers only multi-derivatives with compact supports. $\left(Z\left(T_{r}\right)=\left[x: T_{r}\left(f_{1}, \cdots, f_{r}\right)(x)=0\right.\right.$ for all $\left.f_{1}, \cdots, f_{r}\right]$. Support of $T_{r}=\mathrm{Cl}\left(X-Z\left(T_{r}\right)\right)$.) (Received July 5,1956 .)

\section{2t. G. O. Sabidussi: On the minimum order of graphs with given automorphism group.}

Let $G$ be a finite group. Define $\alpha(G)$ as $\min \alpha_{0}(X)$, the minimum taken over all graphs $X$ whose automorphism group is isomorphic to $G .\left(\alpha_{0}(X)\right.$ is the number of vertices of $X$ ). Earlier estimates for $\alpha(G)$ are improved as follows: If $G$ is a group of order $g$ containing a set $H$ of $h$ generators such that every element of $H$ is of order $\geqq 6$, then $\alpha(G) \leqq g[(\log h) /(2 \log 2)+2]$. (The restriction on the order of the generators can be removed, the result being a more complicated formula). For the cyclical group $C_{n}$ of order $n$ the exact value of $\alpha\left(C_{n}\right)$ is determined. If $G$ is isomorphic to a subgroup of the symmetric group $S_{n}$ of order $n$ !, then $\alpha(G) \leqq n(n+1)(n+6) / 2$. (Received June $4,1956$. )

813t. G. O. Sabidussi: Graph properties independent of the automorphism group.

Given a graph $X$, let $P_{i}, i=0, \cdots, 4$, stand for the following properties of 
$X: P_{0}: X$ is covariant (no automorphism of $X$ leaves all vertices of $X$ invariant); $P_{1}: X$ is of connectivity $n, n \geqq 1 ; P_{2}: X$ is of chromatic number $n, n \geqq 2 ; P_{2}: X$ is regular of degree $n, n \geqq 3 ; P_{4}: X$ contains a Hamiltonian circuit and is spanned by a graph $Y^{\prime}$ homeomorphic to a given connected graph $Y$. The following theorem is proved: Given a finite group $G$ and an integer $i, 1 \leqq i \leqq 4$, there exist infinitely many nonisomorphic connected covariant graphs $X$ such that (a) the automorphism group of $X$ is isomorphic to $G$; (b) $X$ has property $P_{i}$. (Received June 4, 1956.)

\section{J. M. Slye: Spaces with two dimensional rulings.}

If $f$ is a mapping of a space $B$ onto a space $X$, then $G$, the collection of inverse images of points of $X$, is called a ruling of $B$ by $h$, a set of $G$, if each sequential limit set of sets of $G$ that is not a point is homeomorphic to $h$. A point $Q$ of $B$ is called a simple point with respect to the ruling if there exists a connected open set $D$ containing the point $P$, whose inverse image $g$ contains $Q$, and $D-P$ is the sum of two mutually separated sets each of which contains a sequence of points whose inverse images have $Q$ in their limit sets. A point of a set $g$ of $G$ is a singular point if it is a sequential limit point both of simple points and of nonsimple points of $g$. It is shown that a locally compact, locally connected unifoliate metric space $B$ without local cut points, with a ruling $G$ of regular curves with both $h$ and $X$ simplicial and no point that is a limit point of its singular points is a two manifold. (Received July $5,1956$. )

\section{5t. Stephen Smale: Regular curves on Riemannian manifolds.}

A regular curve on a Riemannian manifold is a curve with a continuously turning nontrivial tangent vector. A regular homotopy is a homotopy which at every stage is a regular curve and keeps end points and directions fixed. Let $x_{0}$ be a point of the unit tangent bundle $T$ of a Riemannian manifold $M$. The space (under a certain topology) of all regular curves on $M$ starting at the point and direction determined by $x_{0}$ is denoted by $E$. A map $\pi$ from $E$ onto $T$ is defined by sending a curve into the tangent of its end point at its end point. It is proved that $(E, \pi, T)$ has the covering homotopy property for polyhedra. Using this result and the fact that $E$ is homotopically trivial one gets: There is a 1-1 correspondence between the set of classes (under regular homotopy) of regular curves on $M$ which start and end at the point and direction determined by $x_{0}$, and $\pi_{1}\left(T, x_{0}\right)$. In a sense this is a generalization of the WhitneyGraustein Theorem ( $\mathrm{H}$. Whitney, On regular closed curves in the plane," Compositio Math. vol. 4 (1937) pp. 276-284). (Received June 25, 1956.)

\section{6t. P. M. Swingle and R. P. Hunter: Indecomposable trajectories in phase space.}

Let $D$ be a connected domain in a separable Moore phase space, $S_{m}$, satisfying Axioms 1 and 2. An indecomposable trajectory is an arc-wise indecomposable connexe. The following exist densely in $D$ : a set of $n$ mutually exclusive indecomposable trajectories; an arc-wise trajectory $n$-indecomposable connexe (trajectory union). If an image point moves counter clock-wise densely in a torus, an arc-wise trajectory may or may not be indecomposable: it even may be locally connected. But, if an arcwise trajectory is dense in a locally compact $D$ of $S_{2}$, it is indecomposable. There exists a nonwidely connected hereditarily indecomposable connexe dense in $D$ of $S_{2}$. (If, for $N, H, K$ connected and $N=H+K$, both $H$ and $K$ have different closure than $N$, then $N$ is decomposable: if $N$ is not decomposable, it is indecomposable). (Received May 24, 1956.) 
817t. G. T. Whyburn: Dimension and nondensity preservation of mappings.

The property of a mapping $f(X)=Y$ to preserve nondensity for compact subsets of $X$ is characterized in terms of the lightness kernel $L_{f}$ of $f$ consisting of all $x \in X$ such that $f^{-1} f(x)$ is totally disconnected. These results are applied to show that if $w=f(z)$ is continuous in a region $X$ of the $z$-plane and differentiable at all points of a dense set $f^{-1}\left(Y_{0}\right)$ in $X$ which is the inverse of an open subset $Y_{0}$ of $Y=f(X)$, then $\operatorname{dim} f(K) \leqq 1$ for each compact set $K$ in $X$ of dimension $\leqq 1$. Under the same conditions it is shown that $f$ is strongly quasi-open. (Received July $5,1956$. )

\section{R. E. Zink: A note concerning regular measures.}

Let $X$ be a topological space and let $(X, S, \mu)$ be an associated measure space. Let $f(x)$ be non-negative and measurable $(S)$. Define the measure $\nu$ on $S$ by means of the formula: $\nu(E)=\int_{E} f(x) d \mu(x)$, for all $E$ belonging to $S$. Using the ancient techniques of measure theory, one can easily establish the following remarks: (1) If $\mu$ is inner regular, so also is $\nu$. (2) If $\mu$ is outer regular, then $\nu$ is outer regular if and only if for every set $E$ on which $f$ is integrable: (i) There exists a measurable open set containing $E$ on which $f$ is also integrable. (ii) There exists, for every pair of positive numbers $\epsilon, \delta$, a measurable open set $U$ containing $E-N(f)$ such that $\mu(\{x: f(x)>\delta\} \cap U)<\epsilon$. (Received Iulv $5,1956$.

\section{L. KLEE, JR., Associate Secretary}

\section{RESEARCH PROBLEMS}

\section{Richard Bellman: Approximation theory.}

Consider the differential equation (1) $d x / d t=\Phi(x), x(0)=c$ where $\Phi(x)$ is a continuous function of $x$ satisfying additional conditions ensuring the existence of a unique solution over the interval $0 \leqq t \leqq T$ for $a \leqq c \leqq b$.

Let (2) $d y / d t=\sum_{k=0}^{x} a_{k}(c, t) y^{k}, y(0)=c$, represent an approximation to (1) where the coefficients $a_{k}(c, t)$ are functions to be determined so as to minimize one of the following functionals: (3) (a) $J_{1}=\operatorname{Max}_{0} \leqq t \leqq T|x-y|$, (b) $J_{2}=\int_{0}^{T}(x-y)^{2} d t$, (c) $J_{3}$

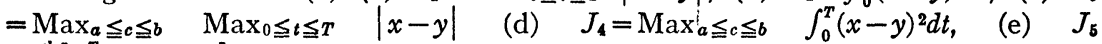
$=\int_{a}^{b}\left[\int_{0}^{T}(x-y)^{2} d t\right] d c$ under the following alternatives: (4) (a) $a_{k}(c, t)$ depends only upon $c$, for $k=0,1, \cdots, K$. (b) $a_{k}(c, t)$ are polynomials of degree $M$ in $t$ with coefficients dependent upon $c$. (The case $K=1$ is the most interesting.)

Consider the analogous problem for systems of the form (5) $d x_{i} / d t=\Phi_{i}\left(x_{1}, x_{2}, \cdots\right.$, $\left.x_{n}\right), x_{i}(0)=c_{i}, i=1,2, \cdots, n$, in the particular case where the approximating equation is (6) $d y_{i} / d t=a_{i}(c)+\sum_{i=1}^{n} b_{i j}(c) y_{j}(t), y_{i}(0)=c_{i}$, and we wish to minimize the functional (7) $\int_{a_{1}}^{b_{1}} \cdots \int_{a_{n}}^{b_{n}}\left[\int_{0}^{r} \sum_{i=0}^{n}\left(x_{i}-y_{i}\right)^{2} d t\right] \Pi_{i} d c_{i}$. (Received August 17, 1956.) 